\title{
Local large deviations principle for occupation measures of the stochastic damped nonlinear wave equation
}

\author{
D. Martirosyan ${ }^{\mathrm{a}}$ and V. Nersesyan ${ }^{\mathrm{b}}$ \\ ${ }^{a}$ Department of Mathematics, University of Cergy-Pontoise, CNRS UMR 8088, 2 avenue Adolphe Chauvin, 95300 Cergy-Pontoise, France. \\ E-mail: Davit.Martirosyan@u-cergy.fr \\ ${ }^{\mathrm{b}}$ Laboratoire de Mathématiques, UMR CNRS 8100, Université de Versailles-Saint-Quentin-en-Yvelines, F-78035 Versailles, France. \\ E-mail:Vahagn.Nersesyan@math.uvsq.fr
}

Received 18 October 2016; revised 2 June 2017; accepted 1 September 2017

\begin{abstract}
We consider the damped nonlinear wave (NLW) equation driven by a noise which is white in time and colored in space. Assuming that the noise is non-degenerate in all Fourier modes, we establish a large deviations principle (LDP) for the occupation measures of the trajectories. The lower bound in the LDP is of a local type, which is related to the weakly dissipative nature of the equation and is a novelty in the context of randomly forced PDE's. The proof is based on an extension of methods developed in (Comm. Pure Appl. Math. 68 (12) (2015) 2108-2143) and (Large deviations and mixing for dissipative PDE's with unbounded random kicks (2014) Preprint) in the case of kick forced dissipative PDE's with parabolic regularization property such as, for example, the Navier-Stokes system and the complex Ginzburg-Landau equations. We also show that a high concentration towards the stationary measure is impossible, by proving that the rate function that governs the LDP cannot have the trivial form (i.e., vanish on the stationary measure and be infinite elsewhere).
\end{abstract}

Résumé. Nous considérons l'équation des ondes non linéaire avec un bruit qui est blanc en temps et coloré en espace. Sous l'hypothèse que le bruit est non dégénéré, nous établissons un principe de grandes déviations (PGD) pour la famille de mesures d'occupation des trajectoires. La borne inférieure dans le PGD est d'un type local, qui est lié à la nature faiblement dissipative de l'équation. La preuve est basée sur une généralisation des méthodes développées dans (Comm. Pure Appl. Math. 68 (12) (2015) 2108-2143) et (Large deviations and mixing for dissipative PDE's with unbounded random kicks (2014) Preprint) pour des EDP paraboliques, comme les équations de Navier-Stokes ou de Ginzburg-Landau complexe, perturbées par une force aléatoire discrète en temps. Nous montrons également que la fonction de taux du PGD n'est pas triviale, ce qui implique qu'une forte concentration vers la mesure stationnaire est impossible.

MSC: 35L70; 35R60; 60B12; 60F10

Keywords: Nonlinear wave equation; White in time noise; Large deviations principle; Coupling method

\section{Introduction}

This paper is devoted to the study of the large deviations principle (LDP) for the occupation measures of the stochastic nonlinear wave (NLW) equation in a bounded domain $D \subset \mathbb{R}^{3}$ with a smooth boundary $\partial D$ :

$$
\begin{aligned}
& \partial_{t}^{2} u+\gamma \partial_{t} u-\Delta u+f(u)=h(x)+\vartheta(t, x),\left.\quad u\right|_{\partial D}=0, \\
& {[u(0), \dot{u}(0)]=\left[u_{0}, u_{1}\right] .}
\end{aligned}
$$

Here $\gamma>0$ is a damping parameter, $h$ is a function in $H_{0}^{1}(D)$, and $f$ is a nonlinear term satisfying some standard dissipativity and growth conditions (see (1.1)-(1.3)). These conditions are satisfied for the classical examples $f(u)=$ 
$\sin u$ and $f(u)=|u|^{\rho} u-\lambda u$, where $\lambda \in \mathbb{R}$ and $\rho \in(0,2)$, coming from the damped sine-Gordon and Klein-Gordon equations. We assume that $\vartheta(t, x)$ is a colored white noise of the form

$$
\vartheta(t, x)=\partial_{t} \xi(t, x), \quad \xi(t, x)=\sum_{j=1}^{\infty} b_{j} \beta_{j}(t) e_{j}(x),
$$

where $\left\{\beta_{j}\right\}$ is a sequence of independent standard Brownian motions, the set of functions $\left\{e_{j}\right\}$ is an orthonormal basis in $L^{2}(D)$ formed by eigenfunctions of the Dirichlet Laplacian with eigenvalues $\left\{\lambda_{j}\right\}$, and $\left\{b_{j}\right\}$ is a sequence of real numbers satisfying

$$
B_{1}:=\sum_{j=1}^{\infty} \lambda_{j} b_{j}^{2}<\infty .
$$

We denote by $\left(\mathfrak{u}_{t}, \mathbb{P}_{\mathfrak{u}}\right), \mathfrak{u}_{t}=\left[u_{t}, \dot{u}_{t}\right]$ the Markov family associated with this stochastic NLW equation and parametrized by the initial condition $\mathfrak{u}=\left[u_{0}, u_{1}\right] \in H_{0}^{1}(D) \times L^{2}(D)$. The exponential ergodicity for this family is established in the paper [21].

The LDP for the occupation measures of randomly forced PDE's has been previously established in $[7,8]$ in the case of the Burgers equation and the Navier-Stokes system, based on some abstract results from [27]. In these papers, the random force is assumed to be rough in the space variable and white in time, i.e., it is of the form $(0.3)$ with the following condition on the coefficients:

$$
c j^{-\alpha} \leq b_{j} \leq C j^{-\frac{1}{2}-\varepsilon}, \quad \frac{1}{2}<\alpha<1, \quad \varepsilon \in\left(0, \alpha-\frac{1}{2}\right], j \geq 1 .
$$

This condition implies that the Markov process associated with the problem has the strong Feller property (SFP). The SFP plays a central role in the arguments of the mentioned three papers. In the case of a spatially regular noise, i.e. when the coefficients $b_{j}$ in (0.5) are allowed to converge to zero sufficiently fast, it is not known if the SFP still holds.

In the case of a perturbation which is a regular random kick force, the LDP is proved in [10,12] for a family of PDE's with parabolic regularization (such as the Navier-Stokes system or the complex Ginzburg-Landau equation). See also [11] for the proof of the LDP and the Gallavotti-Cohen principle in the case of a rough kick force.

Let us explain in a few words the goal of this paper as well as the choice of the model. Our aim is to extend the results and the methods developed in $[10,12]$ under more general assumptions on both stochastic and deterministic parts of the equation. The stochastic NLW equation suits well for implementing this analysis. Indeed, on the one hand it is probably the most representative SPDE of hyperbolic type. The fact that it is only weakly dissipative and lacks a regularizing property allows to cover a wide range of systems with similar properties. On the other hand, it is one of the few equations without a regularizing effect for which the (exponential) ergodicity is known.

In what follows, we shall denote by $\mu$ the stationary measure of the family $\left(\mathfrak{u}_{t}, \mathbb{P}_{\mathfrak{u}}\right)$, and for any bounded continuous function $\psi: H_{0}^{1}(D) \times L^{2}(D) \rightarrow \mathbb{R}$, we shall write $\langle\psi, \mu\rangle$ for the integral of $\psi$ with respect to $\mu$. Let $\mathcal{U}$ be the space of bounded Hölder-continuous functions $\psi: H_{0}^{1}(D) \times L^{2}(D) \rightarrow \mathbb{R}$ depending only on finite-dimensional projections (see (1.10)). We prove the following level-1 LDP for the solutions of problem (0.1), (0.3).

Main Theorem. Assume that conditions (0.4) and (1.1)-(1.3) are verified and $b_{j}>0$ for all $j \geq 1$. Then for any non-constant function $\psi \in \mathcal{U}$, there is $\varepsilon=\varepsilon(\psi)>0$ and a convex function $I^{\psi}: \mathbb{R} \rightarrow \mathbb{R}_{+}$such that, for any ${ }^{1} \mathfrak{u} \in$ $H^{r+1}(D) \times H^{r}(D)$ and any open subset $O$ of the interval $(\langle\psi, \mu\rangle-\varepsilon,\langle\psi, \mu\rangle+\varepsilon)$, we have

$$
\lim _{t \rightarrow \infty} \frac{1}{t} \log \mathbb{P}_{\mathfrak{u}}\left\{\frac{1}{t} \int_{0}^{t} \psi(\mathfrak{u}(\tau)) \mathrm{d} \tau \in O\right\}=-\inf _{\alpha \in O} I^{\psi}(\alpha)
$$

where $r>0$ is a small number. Moreover, limit (0.6) is uniform with respect to $\mathfrak{u}$ in a bounded set of $H^{r+1}(D) \times$ $H^{r}(D)$.

\footnotetext{
${ }^{1}$ Here $H^{r}(D)$ is the domain of the definition of $(-\Delta)^{r / 2}$ (see $\left.(0.7)\right)$.
} 
The proof of this result is obtained by extending the techniques and results introduced in $[10,12]$. According to a local version of the Gärtner-Ellis theorem, relation (0.6) will be established if we show that, for some $\beta_{0}>0$, the following limit exists

$$
Q(\beta)=\lim _{t \rightarrow+\infty} \frac{1}{t} \log \mathbb{E}_{\mathfrak{u}} \exp \left(\int_{0}^{t} \beta \psi\left(\mathfrak{u}_{\tau}\right) \mathrm{d} \tau\right), \quad|\beta|<\beta_{0}
$$

and it is differentiable in $\beta$ on $\left(-\beta_{0}, \beta_{0}\right)$. We show that both properties can be derived from a multiplicative ergodic theorem, which is a convergence result for the Feynman-Kac semigroup of the stochastic NLW equation. A continuous-time version of a criterion established in [10] shows that a multiplicative ergodic theorem holds provided that the following four conditions are satisfied: uniform irreducibility, exponential tightness, growth condition, and uniform Feller property. The smoothness of the noise and the lack of a strong dissipation and of a regularizing property in the equation result in substantial differences in the techniques used to verify these conditions. While in the case of kick-forced models the first two of them are checked directly, they have a rather non-trivial proof in our case, relying on a feedback stabilization result and some subtle estimates for the Sobolev norms of the solutions. Nonetheless, the most involved and highly technical part of the paper remains the verification of the uniform Feller property. Based on the coupling method, its proof is more intricate here mainly due to a more complicated Foiaş-Prodi type estimate for the stochastic NLW equation. An important difference compared with the parabolic case is that we get a uniform Feller property only for potentials that have a sufficiently small oscillation, and this is the main reason why the LDP established in this paper is of a local type.

We also establish a more general result of level-2 type in Theorem 1.1 and an abstract criterion given in Section A.1. These results are slightly different from the standard Donsker-Varadhan form (e.g., see Theorem 3 in [6]), since here the LDP is proved to hold locally on some part of the phase space. Another abstract result in this direction can be found in Theorem 4.5.20 in [5]. The question of global LDP for our system is open and, certainly, deserves further investigation.

Let us mention that there is a substantial amount of literature studying the local LDP of level-1 type for finitedimensional deterministic and stochastic dynamical systems (e.g., see [9,13,15,25]). The papers [15] and [2] provide an example of a Doeblin chain with good mixing properties for which the local LDP holds whereas the global one fails.

The paper is organized as follows. We formulate in Section 1 the second main result of this paper on the level-2 LDP for the NLW equation and, by using a local version of Kifer's criterion, we reduce its proof to a multiplicative ergodic theorem. Section 2 is devoted to the derivation of the Main Theorem. In Sections 3 and 4, we are checking the conditions of an abstract result about the convergence of generalized Markov semigroups. In Section 5, we prove the exponential tightness property and provide some estimates for the growth of Sobolev norms of the solutions. The multiplicative ergodic theorem is established in Section 6. In the Appendix, we prove the local version of Kifer's criterion, the abstract convergence result for the semigroups, and some other technical results which are used throughout the paper.

\section{Notation}

For a Banach space $X$, we denote by $B_{X}(a, R)$ the closed ball in $X$ of radius $R$ centered at $a$. In the case when $a=0$, we write $B_{X}(R)$. For any function $V: X \rightarrow \mathbb{R}$, we set $\operatorname{Osc}_{X}(V):=\sup _{X} V-\inf _{X} V$. We use the following spaces:

$L^{\infty}(X)$ is the space of bounded measurable functions $\psi: X \rightarrow \mathbb{R}$ endowed with the norm $\|\psi\|_{\infty}=\sup _{u \in X}|\psi(u)|$. $C_{b}(X)$ is the space of continuous functions $\psi \in L^{\infty}(X)$, and $C_{+}(X)$ is the space of positive continuous functions $\psi: X \rightarrow \mathbb{R}$.

$C_{b}^{q}(X), q \in(0,1]$ is the space of functions $f \in C_{b}(X)$ for which the following norm is finite

$$
\|\psi\|_{C_{b}^{q}}=\|\psi\|_{\infty}+\sup _{u \neq v} \frac{|\psi(u)-\psi(v)|}{\|u-v\|^{q}} .
$$

$\mathcal{M}(X)$ is the vector space of signed Borel measures on $X$ with finite total mass. For $\mu \in \mathcal{M}(X)$ and $\psi \in C_{b}(X)$, we denote $\langle\psi, \mu\rangle=\int_{X} \psi(u) \mu(\mathrm{d} u)$. The space $\mathcal{M}(X)$ is endowed with the topology of the weak convergence, i.e. 
the weakest topology such that $\mu \mapsto\langle f, \mu\rangle$ is continuous for any $f \in C_{b}(X) . \mathcal{M}_{+}(X) \subset \mathcal{M}(X)$ is the cone of non-negative measures.

$\mathcal{P}(X)$ is the set of probability Borel measures on $X$. If $\mu_{1}, \mu_{2} \in \mathcal{P}(X)$, we set

$$
\left|\mu_{1}-\mu_{2}\right|_{\mathrm{var}}=\sup \left\{\left|\mu_{1}(\Gamma)-\mu_{2}(\Gamma)\right|: \Gamma \in \mathcal{B}(X)\right\},
$$

where $\mathcal{B}(X)$ is the Borel $\sigma$-algebra of $X$.

For any measurable function $\mathfrak{w}: X \rightarrow[1,+\infty]$, let $C_{\mathfrak{w}}(X)$ (respectively, $L_{\mathfrak{w}}^{\infty}(X)$ ) be the space of continuous (measurable) functions $\psi: X \rightarrow \mathbb{R}$ such that $|\psi(u)| \leq C \mathfrak{w}(u)$ for all $u \in X$. We endow $C_{\mathfrak{w}}(X)$ and $L_{\mathfrak{w}}^{\infty}(X)$ with the seminorm

$$
\|\psi\|_{L_{\mathfrak{w}}^{\infty}}=\sup _{u \in X} \frac{|\psi(u)|}{\mathfrak{w}(u)} .
$$

$\mathcal{P}_{\mathfrak{w}}(X)$ is the space of measures $\mu \in \mathcal{P}(X)$ such that $\langle\mathfrak{w}, \mu\rangle<\infty$.

For an open set $D$ of $\mathbb{R}^{3}$, we introduce the following function spaces:

$L^{p}=L^{p}(D)$ is the Lebesgue space of measurable functions whose $p$ th power is integrable. In the case $p=2$ the corresponding norm is denoted by $\|\cdot\|$.

$H^{r}=H^{r}(D), r \geq 0$ is the domain of definition of the operator $(-\Delta)^{r / 2}$ endowed with the norm $\|\cdot\|_{r}$ :

$$
H^{r}=\mathfrak{D}\left((-\Delta)^{r / 2}\right)=\left\{u=\sum_{j=1}^{\infty} u_{j} e_{j} \in L^{2}:\|u\|_{r}^{2}:=\sum_{j=1}^{\infty} \lambda_{j}^{s} u_{j}^{2}<\infty\right\}
$$

In particular, $H^{1}$ coincides with $H_{0}^{1}(D)$, the space of functions in the Sobolev space of order 1 that vanish at the boundary. We denote by $H^{-r}$ the dual of $H^{r}$.

\section{Level-2 LDP for the NLW equation}

\subsection{Stochastic NLW equation and its mixing properties}

In this subsection we give the precise hypotheses on the nonlinearity and recall a result on the property of exponential mixing for the Markov family associated with the flow of $(0.1)$. We shall assume that the function $f$ satisfies the following regularity and growth conditions

$$
f \in C^{2}(\mathbb{R}), \quad f(0)=0, \quad\left|f^{\prime \prime}(u)\right| \leq C\left(|u|^{\rho-1}+1\right), \quad u \in \mathbb{R},
$$

for some positive constants $C$ and $\rho<2$, and the dissipativity conditions

$$
\begin{aligned}
& F(u) \geq C^{-1}\left|f^{\prime}(u)\right|^{\frac{\rho+2}{\rho}}-v u^{2}-C, \\
& f(u) u-F(u) \geq-v u^{2}-C,
\end{aligned}
$$

where $F$ is a primitive of $f, v$ is a positive number less than $\left(\lambda_{1} \wedge \gamma\right) / 8$. Let us note that inequality (1.2) is slightly more restrictive than the one used in [21]; this hypothesis allows us to establish the exponential tightness property (see Section 5.1). We consider the NLW equation in the phase space $\mathcal{H}=H^{1} \times L^{2}$ endowed with the norm

$$
|\mathfrak{u}|_{\mathcal{H}}^{2}=\left\|u_{1}\right\|_{1}^{2}+\left\|u_{2}+\alpha u_{1}\right\|^{2}, \quad \mathfrak{u}=\left[u_{1}, u_{2}\right] \in \mathcal{H},
$$

where $\alpha=\alpha(\gamma)>0$ is a small parameter. Under the above conditions, for any initial data $\mathfrak{u}_{0}=\left[u_{0}, u_{1}\right] \in \mathcal{H}$, there is a unique solution (or a flow) $\mathfrak{u}_{t}=\mathfrak{u}\left(t ; \mathfrak{u}_{0}\right)=\left[u_{t}, \dot{u}_{t}\right]$ of problem $(0.1)-(0.3)$ in $\mathcal{H}$ (see Section 7.2 in [3]). For any 
$r \in \mathbb{R}$, let $\mathcal{H}^{r}$ denote the space $H^{r+1} \times H^{r}$ endowed with the norm

$$
|\mathfrak{u}|_{\mathcal{H}^{r}}^{2}=\left\|u_{1}\right\|_{r+1}^{2}+\left\|u_{2}+\alpha u_{1}\right\|_{r}^{2}, \quad \mathfrak{u}=\left[u_{1}, u_{2}\right] \in \mathcal{H}^{r}
$$

with the same $\alpha$ as in (1.4). If $\mathfrak{u}_{0} \in \mathcal{H}^{r}$ and $0<r<1-\rho / 2$, the solution $\mathfrak{u}\left(t ; \mathfrak{u}_{0}\right)$ belongs ${ }^{2}$ to $\mathcal{H}^{r}$ almost surely.

Let $\left(\mathfrak{u}_{t}, \mathbb{P}_{\mathfrak{u}}\right)$ be the Markov family associated with $(0.1)$, and let $P_{t}(\mathfrak{u}, \Gamma)=\mathbb{P}_{\mathfrak{u}}\left\{\mathfrak{u}_{t} \in \Gamma\right\}$ be its transition function. Recall that a measure $\mu \in \mathcal{P}(\mathcal{H})$ is said to be stationary if

$$
\mu(\Gamma)=\int_{\mathcal{H}} P_{t}(\mathfrak{u}, \Gamma) \mu(\mathrm{d} \mathfrak{u}) \quad \text { for any } t \geq 0 .
$$

By Theorem 5.3 in [21], we know that, if conditions (1.1)-(1.3) are verified and $b_{j}>0, j \geq 1$ is a sequence satisfying (0.4), then the family $\left(\mathfrak{u}_{t}, \mathbb{P}_{\mathfrak{u}}\right)$ has a unique stationary measure $\mu \in \mathcal{P}(\mathcal{H})$.

\subsection{The statement of the result}

Before giving the formulation of the main result of this section, let us introduce some notation and recall some basic definitions from the theory of LDP (see [5]). For any $\mathfrak{u} \in \mathcal{H}$, we define the following family of occupation measures

$$
\zeta_{t}=\frac{1}{t} \int_{0}^{t} \delta_{\mathfrak{u}_{\tau}} \mathrm{d} \tau, \quad t>0,
$$

where $\mathfrak{u}_{\tau}:=\mathfrak{u}(\tau ; \mathfrak{u})$ and $\delta_{\mathfrak{v}}$ is the Dirac measure concentrated at $\mathfrak{v} \in \mathcal{H}$. For any $V \in C_{b}(\mathcal{H})$ and $R>0$, we set

$$
Q_{R}(V)=\limsup _{t \rightarrow+\infty} \frac{1}{t} \log \sup _{\mathfrak{u} \in X_{R}} \mathbb{E}_{\mathfrak{u}} \exp \left(t\left\langle V, \zeta_{t}\right\rangle\right),
$$

where $X_{R}:=B_{\mathcal{H}^{r}}(R), r \in(0,1-\rho / 2)$. Then $Q_{R}: C_{b}(\mathcal{H}) \rightarrow \mathbb{R}$ is a convex 1-Lipschitz function, and its Legendre transform is given by

$$
I_{R}(\sigma):= \begin{cases}\sup _{V \in C_{b}(\mathcal{H})}\left(\langle V, \sigma\rangle-Q_{R}(V)\right) & \text { for } \sigma \in \mathcal{P}(\mathcal{H}), \\ +\infty & \text { for } \sigma \in \mathcal{M}(\mathcal{H}) \backslash \mathcal{P}(\mathcal{H}) .\end{cases}
$$

The function $I_{R}: \mathcal{M}(\mathcal{H}) \rightarrow[0,+\infty]$ is convex lower semicontinuous in the weak topology, and $Q_{R}$ can be reconstructed from $I_{R}$ by the formula

$$
Q_{R}(V)=\sup _{\sigma \in \mathcal{P}(\mathcal{H})}\left(\langle V, \sigma\rangle-I_{R}(\sigma)\right) \quad \text { for any } V \in C_{b}(\mathcal{H}) .
$$

Note that, for any $\sigma \in \mathcal{M}(\mathcal{H}), I_{R}(\sigma)$ is non-increasing in $R$, so we can set

$$
I(\sigma)=\lim _{R \rightarrow+\infty} I_{R}(\sigma) .
$$

We denote by $\mathcal{V}$ the set of functions $V \in C_{b}(\mathcal{H})$ satisfying the following two properties.

Property 1. For any $R>0$ and $\mathfrak{u} \in X_{R}$, the following limit exists (called pressure function)

$$
Q(V)=\lim _{t \rightarrow+\infty} \frac{1}{t} \log \mathbb{E}_{\mathfrak{u}} \exp \left(\int_{0}^{t} V\left(\mathfrak{u}_{\tau}\right) \mathrm{d} \tau\right)
$$

and does not depend on the initial condition $\mathfrak{u}$ and the number $R>0$. Moreover, this limit is uniform with respect to $\mathfrak{u} \in X_{R}$.

\footnotetext{
${ }^{2}$ Some estimates for the $\mathcal{H}^{r}$-norm of the solutions are given in Section 5.2.
} 
Property 2. There is a unique measure $\sigma_{V} \in \mathcal{P}(\mathcal{H})$ (called equilibrium state) satisfying the equality

$$
Q(V)=Q_{R}(V)=\left\langle V, \sigma_{V}\right\rangle-I_{R}\left(\sigma_{V}\right)
$$

for any $R>0$.

A mapping $I: \mathcal{M}(\mathcal{H}) \rightarrow[0,+\infty]$ is a good rate function if for any $a \geq 0$ the level set $\{\sigma \in \mathcal{M}(\mathcal{H}): I(\sigma) \leq a\}$ is compact. A good rate function $I$ is non-trivial if the effective domain $D_{I}:=\{\sigma \in \mathcal{M}(\mathcal{H}): I(\sigma)<\infty\}$ is not a singleton. Finally, we shall denote by $\mathcal{U}$ the set of functions $V \in C_{b}(\mathcal{H})$ for which there is a number $q \in(0,1]$, an integer $N \geq 1$, and a function $F \in C_{b}^{q}\left(\mathcal{H}_{N}\right)$ such that

$$
V(\mathfrak{u})=F\left(P_{N} \mathfrak{u}\right), \quad \mathfrak{u} \in \mathcal{H},
$$

where $\mathcal{H}_{N}:=H_{N} \times H_{N}, H_{N}:=\operatorname{span}\left\{e_{1}, \ldots, e_{N}\right\}$, and $P_{N}$ is the orthogonal projection in $\mathcal{H}$ onto $\mathcal{H}_{N}$. Given a number $\delta>0, \mathcal{U}_{\delta}$ is the subset of functions $V \in \mathcal{U}$ satisfying $\operatorname{Osc}(V)<\delta$.

Theorem 1.1. Under the conditions of the Main Theorem, there is a number $\delta>0$ such that $\mathcal{U}_{\delta} \subset \mathcal{V}$. The function $I: \mathcal{M}(\mathcal{H}) \rightarrow[0,+\infty]$ defined by (1.8) is a non-trivial good rate function, and for any $R>0$, the family $\left\{\zeta_{t}, t>0\right\}$ satisfies the following local LDP.

Upper bound. For any closed set $F \subset \mathcal{P}(\mathcal{H})$, we have

$$
\limsup _{t \rightarrow \infty} \frac{1}{t} \log \sup _{\mathfrak{u} \in X_{R}} \mathbb{P}_{\mathfrak{u}}\left\{\zeta_{t} \in F\right\} \leq-I(F) .
$$

Lower bound. For any open set $G \subset \mathcal{P}(\mathcal{H})$, we have

$$
\liminf _{t \rightarrow \infty} \frac{1}{t} \log \inf _{\mathfrak{u} \in X_{R}} \mathbb{P}_{\mathfrak{u}}\left\{\zeta_{t} \in G\right\} \geq-I(\mathcal{W} \cap G)
$$

$\operatorname{Here}^{3} I(\Gamma):=\inf _{\sigma \in \Gamma} I(\sigma)$ for $\Gamma \subset \mathcal{P}(\mathcal{H})$ and $\mathcal{W}:=\left\{\sigma_{V}: V \in \mathcal{U}_{\delta}\right\}$, where $\sigma_{V}$ is the equilibrium state corresponding to $V$.

This theorem is proved in the next subsection, using a multiplicative ergodic theorem and a local version of Kifer's criterion for LDP. Then in Section 2, we combine it with a local version of the Gärtner-Ellis theorem to establish the Main Theorem.

\subsection{Reduction to a multiplicative ergodic theorem}

In this subsection we reduce the proof of Theorem 1.1 to some properties related to the large-time behavior of the Feynman-Kac semigroup defined by

$$
\mathfrak{P}_{t}^{V} \psi(\mathfrak{u})=\mathbb{E}_{\mathfrak{u}}\left\{\psi\left(\mathfrak{u}_{t}\right) \exp \left(\int_{0}^{t} V\left(\mathfrak{u}_{\tau}\right) \mathrm{d} \tau\right)\right\}
$$

For any $V \in C_{b}(\mathcal{H})$ and $t \geq 0$, the application $\mathfrak{P}_{t}^{V}$ maps $C_{b}(\mathcal{H})$ into itself. Let us denote by $\mathfrak{P}_{t}^{V *}: \mathcal{M}_{+}(\mathcal{H}) \rightarrow$ $\mathcal{M}_{+}(\mathcal{H})$ its dual semigroup, and recall that a measure $\mu \in \mathcal{P}(\mathcal{H})$ is an eigenvector if there is $\lambda \in \mathbb{R}$ such that $\mathfrak{P}_{t}^{V *} \mu=$ $\lambda^{t} \mu$ for any $t>0$.

Let us define a function $\mathfrak{w}: \mathcal{H} \rightarrow[0, \infty]$ by

$$
\mathfrak{w}(\mathfrak{u})=1+|\mathfrak{u}|_{\mathcal{H}^{r}}^{2}+\mathcal{E}^{4}(\mathfrak{u}),
$$

\footnotetext{
${ }^{3}$ The infimum over an empty set is equal to $+\infty$.
} 
which will play the role of the weight function. Here

$$
\mathcal{E}(\mathfrak{u})=|\mathfrak{u}|_{\mathcal{H}}^{2}+2 \int_{D} F\left(u_{1}\right) \mathrm{d} x, \quad \mathfrak{u}=\left[u_{1}, u_{2}\right] \in \mathcal{H}
$$

is the energy functional of the NLW equation. From (5.24) with $m=1$ it follows that $\mathfrak{P}_{t}^{V}$ maps ${ }^{4} C_{\mathfrak{w}}\left(\mathcal{H}^{r}\right)$ into itself (note that $\mathfrak{w}_{1}=\mathfrak{w}$ in (5.24)). We shall say that a function $h \in C_{\mathfrak{w}}\left(\mathcal{H}^{r}\right)$ is an eigenvector for the semigroup $\mathfrak{P}_{t}^{V}$ if $\mathfrak{P}_{t}^{V} h(\mathfrak{u})=\lambda^{t} h(\mathfrak{u})$ for any $\mathfrak{u} \in \mathcal{H}^{r}$ and $t>0$. Then we have the following theorem.

Theorem 1.2. Under the conditions of the Main Theorem, there is $\delta>0$ such that the following assertions hold for any $V \in \mathcal{U}_{\delta}$.

Existence and uniqueness. The semigroup $\mathfrak{P}_{t}^{V *}$ admits a unique eigenvector $\mu_{V} \in \mathcal{P}_{\mathfrak{w}}(\mathcal{H})$ corresponding to an eigenvalue $\lambda_{V}>0$. Moreover, for any $m \geq 1$, we have

$$
\int_{\mathcal{H}}\left[|\mathfrak{u}|_{\mathcal{H}^{r}}^{m}+\exp (\varkappa \mathcal{E}(\mathfrak{u}))\right] \mu_{V}(\mathrm{~d} \mathfrak{u})<\infty
$$

where $\varkappa:=(2 \alpha)^{-1} B_{0}$ and $B_{0}:=\sum b_{j}^{2}$. The semigroup $\mathfrak{P}_{t}^{V}$ admits a unique eigenvector $h_{V} \in C_{\mathfrak{w}}\left(\mathcal{H}^{r}\right) \cap C_{+}\left(\mathcal{H}^{r}\right)$ corresponding to $\lambda_{V}$ normalized by the condition $\left\langle h_{V}, \mu_{V}\right\rangle=1$.

Convergence. For any $\psi \in C_{\mathfrak{w}}\left(\mathcal{H}^{r}\right), v \in \mathcal{P}_{\mathfrak{w}}(\mathcal{H})$, and $R>0$, we have

$$
\begin{aligned}
& \lambda_{V}^{-t} \mathfrak{P}_{t}^{V} \psi \rightarrow\left\langle\psi, \mu_{V}\right\rangle h_{V} \quad \text { in } C_{b}\left(X_{R}\right) \cap L^{1}\left(\mathcal{H}, \mu_{V}\right) \text { as } t \rightarrow \infty, \\
& \lambda_{V}^{-t} \mathfrak{P}_{t}^{V *} v \rightarrow\left\langle h_{V}, v\right\rangle \mu_{V} \quad \text { in } \mathcal{M}_{+}(\mathcal{H}) \text { as } t \rightarrow \infty
\end{aligned}
$$

This result is proved in Section 6. Here we apply it to establish Theorem 1.1.

Proof of Theorem 1.1. Step 1: Proof of the inclusion $\mathcal{U}_{\delta} \subset \mathcal{V}$. Let $\delta>0$ be the constant in Theorem 1.2. Taking $\psi=1$ in (1.15), we get Property 1 with $Q_{R}(V):=\log \lambda_{V}$ for any $V \in \mathcal{U}_{\delta}$ (in particular, $Q(V):=Q_{R}(V)$ does not depend on $R$ ).

Property 2 is deduced from limit (1.15) in the same way as in [10]. Indeed, for any $V \in \mathcal{U}_{\delta}$, we introduce the semigroup

$$
\mathfrak{S}_{t}^{V, F} \psi(\mathfrak{u})=\lambda_{V}^{-t} h_{V}^{-1} \mathfrak{P}_{t}^{V+F}\left(h_{V} \psi\right)(\mathfrak{u}), \quad \psi, F \in C_{b}(\mathcal{H}), t \geq 0,
$$

the function

$$
Q_{R}^{V}(F):=\limsup _{t \rightarrow+\infty} \frac{1}{t} \log \sup _{\mathfrak{u} \in X_{R}} \log \left(\mathfrak{S}_{t}^{V, F} \mathbf{1}\right)(\mathfrak{u})
$$

and the Legendre transform $I_{R}^{V}: \mathcal{M}(\mathcal{H}) \rightarrow[0,+\infty]$ of $Q_{R}^{V}(\cdot)$. The arguments of Section 5.7 of [10] show that $\sigma \in \mathcal{P}(\mathcal{H})$ is an equilibrium state for $V$ if and only if $I_{R}^{V}(\sigma)=0$. So the uniqueness follows from the following result which is a continuous-time version of Proposition 7.5 in [10]. Its proof is given in the Appendix.

Proposition 1.3. For any $V \in \mathcal{U}_{\delta}$ and $R>0$, the measure $\sigma_{V}=h_{V} \mu_{V}$ is the unique zero of $I_{R}^{V}$.

Step 2: Upper and lower bounds. We apply Theorem A.1 to prove estimates (1.11) and (1.12). Let us consider the following totally ordered set $(\Theta, \prec)$, where $\Theta=\mathbb{R}_{+}^{*} \times X_{R}$ and $\prec$ is a relation defined by $\left(t_{1}, \mathfrak{u}_{1}\right) \prec\left(t_{2}, \mathfrak{u}_{2}\right)$ if and only if $t_{1} \leq t_{2}$. For any $\theta=(t, \mathfrak{u}) \in \Theta$, we set $r_{\theta}:=t$ and $\zeta_{\theta}:=\zeta_{t}$, where $\zeta_{t}$ is the random probability measure given by (1.5) defined on the probability space $\left(\Omega_{\theta}, \mathcal{F}_{\theta}, \mathbb{P}_{\theta}\right):=\left(\Omega, \mathcal{F}, \mathbb{P}_{\mathfrak{u}}\right)$. Note that a family $\left\{x_{\theta} \in \mathbb{R}, \theta \in \Theta\right\}$ converges if and only if it converges uniformly with respect to $\mathfrak{u} \in X_{R}$ as $t \rightarrow+\infty$. We have that (A.1) holds with $Q=Q_{R}$ for

\footnotetext{
${ }^{4}$ When we write $C_{\mathfrak{w}}\left(\mathcal{H}^{r}\right)$ or $C\left(X_{R}\right)$, the sets $\mathcal{H}^{r}$ and $X_{R}$ are assumed to be endowed with the topology induced by $\mathcal{H}$.
} 
any $V \in C_{b}(\mathcal{H})$, and for any $V \in \mathcal{V}$, Properties 1 and 2 imply limit (A.3) and the uniqueness of the equilibrium state. Let $\left\{V_{k}\right\} \subset \mathcal{V}_{1}:=\mathcal{U}^{\delta}$ be a sequence which defines the weak topology in $\mathcal{P}(X)$, then $\mathcal{V}_{1} \cap \operatorname{span}\left\{V_{1}, \ldots, V_{n}\right\}$ is open for any $n \geq 1$. It remains to check the following condition, which we postpone to Section 5 .

Exponential tightness. There is a function $\Phi: \mathcal{H} \rightarrow[0,+\infty]$ whose level sets $\{\mathfrak{u} \in \mathcal{H}: \Phi(\mathfrak{u}) \leq a\}$ are compact for any $a \geq 0$ and

$$
\mathbb{E}_{\mathfrak{u}} \exp \left(\int_{0}^{t} \Phi\left(\mathfrak{u}_{\tau}\right) \mathrm{d} \tau\right) \leq C e^{c t}, \quad \mathfrak{u} \in X_{R}, t>0
$$

for some positive constants $C$ and $c$.

Theorem A.1 implies that $I_{R}$ is a good rate function and the following two inequalities hold for any closed set $F \subset$ $\mathcal{P}(\mathcal{H})$ and open set $G \subset \mathcal{P}(\mathcal{H})$

$$
\begin{aligned}
& \limsup _{\theta \in \Theta} \frac{1}{r_{\theta}} \log \mathbb{P}_{\theta}\left\{\zeta_{\theta} \in F\right\} \leq-I_{R}(F), \\
& \liminf _{\theta \in \Theta} \frac{1}{r_{\theta}} \log \mathbb{P}_{\theta}\left\{\zeta_{\theta} \in G\right\} \geq-I_{R}(\mathcal{W} \cap G) .
\end{aligned}
$$

From (1.8) it follows that $I$ is also a good rate function. Let us note that $I_{R}\left(\sigma_{V}\right)$ does not depend on $R$ for $V \in \mathcal{V}$. Indeed, this follows from (1.9). Thus the quantity $I_{R}(\mathcal{W} \cap G)$ does not depend on $R$ as well, so $I_{R}(\mathcal{W} \cap G)=$ $I(\mathcal{W} \cap G)$. On the other hand, $I(F) \leq I_{R}(F)$ for any $R>0$. Combining this with (1.20), (1.21), and the inequalities

$$
\begin{gathered}
\limsup _{\theta \in \Theta} \frac{1}{r_{\theta}} \log \mathbb{P}_{\theta}\left\{\zeta_{\theta} \in F\right\}=\limsup _{t \rightarrow \infty} \frac{1}{t} \log \sup _{\mathfrak{u} \in X_{R}} \mathbb{P}_{\mathfrak{u}}\left\{\zeta_{t} \in F\right\}, \\
\liminf _{\theta \in \Theta} \frac{1}{r_{\theta}} \log \mathbb{P}_{\theta}\left\{\zeta_{\theta} \in G\right\}=\liminf _{t \rightarrow \infty} \frac{1}{t} \log \inf _{\mathfrak{u} \in X_{R}} \mathbb{P}_{\mathfrak{u}}\left\{\zeta_{t} \in G\right\},
\end{gathered}
$$

we obtain (1.11) and (1.12).

Step 3: Non-triviality of $I$. We argue by contradiction. Let us assume that $D_{I}$ is a singleton. Then so is $D_{I_{R}}$ for any $R>0$. By Proposition 1.3 with $V=\mathbf{0}$, we have that the stationary measure $\mu$ is the unique zero ${ }^{5}$ of $I_{R}$, so $D_{I_{R}}=\{\mu\}$. Then (1.7) implies that $Q(V)=\langle V, \mu\rangle$ for any $V \in C_{b}(\mathcal{H})$. Let us choose any non-constant $V \in \mathcal{U}_{\delta}$ such that $\langle V, \mu\rangle=0$. Then $Q(V)=0$, and limit (1.15) with $\psi=\mathbf{1}$ implies that $\lambda_{V}=e^{Q(V)}=1$ and

$$
\sup _{t \geq 0} \mathbb{E}_{0} \exp \left(\int_{0}^{t} V\left(\mathfrak{u}_{\tau}\right) \mathrm{d} \tau\right)<\infty,
$$

where $\mathbb{E}_{0}$ means that we consider the trajectory issued from the origin. Combining this with the central limit theorem (see Theorem 2.5 in [21] and Theorem 4.1.8 and Proposition 4.1.4 in [19]), we get $V=\mathbf{0}$. This contradicts the assumption that $V$ is non-constant and completes the proof of Theorem 1.1.

\section{Proof of the Main Theorem}

Step 1: Proof in the case $\psi \in \mathcal{U}$. For any $R>0$ and non-constant $\psi \in \mathcal{U}$, we denote

$$
I_{R}^{\psi}(p)=\inf \left\{I_{R}(\sigma):\langle\psi, \sigma\rangle=p, \sigma \in \mathcal{P}(\mathcal{H})\right\}, \quad p \in \mathbb{R},
$$

where $I_{R}$ is given by (1.6). Then $Q_{R}(\beta \psi)$ is convex in $\beta \in \mathbb{R}$, and using (1.7), it is straightforward to check that

$$
Q_{R}(\beta \psi)=\sup _{p \in \mathbb{R}}\left(\beta p-I_{R}^{\psi}(p)\right) \quad \text { for } \beta \in \mathbb{R} .
$$

\footnotetext{
${ }^{5}$ Note that when $V=\mathbf{0}$, we have $\lambda_{V}=1, h_{V}=\mathbf{1}, I_{R}^{V}=I_{R}$, and $\mu_{V}=\mu$.
} 
By well-known properties of convex functions of a real variable (e.g., see [26]), $Q_{R}(\beta \psi)$ is differentiable in $\beta \in \mathbb{R}$, except possibly on a countable set, the right and left derivatives $D^{+} Q_{R}(\beta \psi)$ and $D^{-} Q_{R}(\beta \psi)$ exist at any $\beta$ and $D^{-} Q_{R}(\beta \psi) \leq D^{+} Q_{R}(\beta \psi)$. Moreover, the following equality holds for some $\beta, p \in \mathbb{R}$

$$
Q_{R}(\beta \psi)=\beta p-I_{R}^{\psi}(p)
$$

if and only if $p \in\left[D^{-} Q_{R}(\beta \psi), D^{+} Q_{R}(\beta \psi)\right]$. Let us set $\beta_{0}:=\delta /\left(4\|\psi\|_{\infty}\right)$, where $\delta>0$ is the constant in Theorem 1.1. Then for any $|\beta| \leq \beta_{0}$, we have $\beta \psi \in \mathcal{U}_{\delta} \subset \mathcal{V}$ and $Q_{R}(\beta \psi)$ does not depend on $R>0$; we set $Q(\beta \psi):=Q_{R}(\beta \psi)$. Let us show that $D^{-} Q(\beta \psi)=D^{+} Q(\beta \psi)$ for any $|\beta|<\beta_{0}$, i.e., $Q(\beta \psi)$ is differentiable at $\beta$. Indeed, assume that $p_{1}, p_{2} \in\left[D^{-} Q(\beta \psi), D^{+} Q(\beta \psi)\right]$. Then equality (2.1) holds with $p=p_{i}, i=1,2$. As $I_{R}$ is a good rate function, there are measures $\sigma_{i} \in \mathcal{P}(\mathcal{H})$ such that $\left\langle\psi, \sigma_{i}\right\rangle=p_{i}$ and $I_{R}\left(\sigma_{i}\right)=I_{R}^{\psi}\left(p_{i}\right), i=1,2$. Thus

$$
Q(\beta \psi)=\beta p_{i}-I_{R}^{\psi}\left(p_{i}\right)=\left\langle\beta \psi, \sigma_{i}\right\rangle-I_{R}\left(\sigma_{i}\right),
$$

i.e., $\sigma_{1}$ and $\sigma_{2}$ are equilibrium states corresponding to $V=\beta \psi$. As $\beta \psi \in \mathcal{V}$, from Property 2 we derive that $\sigma_{1}=\sigma_{2}$, hence $p_{1}=p_{2}$. Thus $Q(\beta \psi)$ is differentiable at $\beta$ for any $|\beta|<\beta_{0}$. Let us define the convex function

$$
Q^{\psi}(\beta):= \begin{cases}Q(\beta \psi), & \text { for }|\beta| \leq \beta_{0}, \\ +\infty, & \text { for }|\beta|>\beta_{0},\end{cases}
$$

and its Legendre transform

$$
I^{\psi}(p):=\sup _{\beta \in \mathbb{R}}\left(\beta p-Q^{\psi}(\beta)\right) \text { for } p \in \mathbb{R} .
$$

Then $I^{\psi}$ is a finite convex function not depending on $R>0$. As $Q^{\psi}(\beta)$ is differentiable at any $|\beta|<\beta_{0}$ and (A.3) holds with $Q=Q^{\psi}(\beta)$ (with respect to the directed set $(\Theta, \prec)$ defined in the proof of Theorem 1.1), we see that the conditions of Theorem A.5 in [13] are satisfied. ${ }^{6}$ Hence, we have (0.6) for any open subset $O$ of the interval $J^{\psi}:=\left(D^{+} Q^{\psi}\left(-\beta_{0}\right), D^{-} Q^{\psi}\left(\beta_{0}\right)\right)$.

Step 2: The interval $J^{\psi}$. Let us show that if $\psi \in \mathcal{U}$ is non-constant, then the interval $J^{\psi}=\left(D^{+} Q^{\psi}\left(-\beta_{0}\right)\right.$, $\left.D^{-} Q^{\psi}\left(\beta_{0}\right)\right)$ is non-empty and contains the point $\langle\psi, \mu\rangle$. Clearly we can assume that $\langle\psi, \mu\rangle=0$. As $Q^{\psi}(0)=0$, it is sufficient to show that $\beta=0$ is the only point of the interval $\left[-\beta_{0}, \beta_{0}\right]$, where $Q^{\psi}(\beta)$ vanishes. Assume the opposite. Then, replacing $\psi$ by $-\psi$ if needed, we can suppose that there is $\beta \in\left(0, \beta_{0}\right]$ such that $Q^{\psi}(\beta)=0$. As in Step 3 of Theorem 1.1, this implies

$$
\sup _{t \geq 0} \mathbb{E}_{0} \exp \left(\beta \int_{0}^{t} \psi\left(\mathfrak{u}_{\tau}\right) \mathrm{d} \tau\right)<\infty
$$

and $\psi \equiv 0$. This contradicts our assumption that $\psi$ is non-constant and completes the proof of the Main Theorem.

\section{Checking conditions of Theorem A.4}

The proof of Theorem 1.2 is based on an application of Theorem A.4. In this section, we verify the growth condition, the uniform irreducibility property, and the existence of an eigenvector for the following generalized Markov family of transition kernels (see Definition A.3)

$$
P_{t}^{V}(\mathfrak{u}, \Gamma)=\left(\mathfrak{P}_{t}^{V *} \delta_{\mathfrak{u}}\right)(\Gamma), \quad V \in C_{b}(\mathcal{H}), \Gamma \in \mathcal{B}(\mathcal{H}), \mathfrak{u} \in \mathcal{H}, t \geq 0
$$

in the phase space $X=\mathcal{H}$ endowed with a sequence of compacts $X_{R}=B \mathcal{H}^{r}(R), R \geq 1$ and a weight function $\mathfrak{w}$ defined by (1.13). The uniform Feller property is the most delicate condition to check in Theorem A.4, it will be established in Section 4. In the rest of the paper, we shall always assume that the hypotheses of Theorem 1.1 are fulfilled.

\footnotetext{
${ }^{6}$ Theorem A.5 in [13] is stated in the case $\Theta=\mathbb{R}_{+}$. However, the proof presented there remains valid for random variables indexed by a directed set.
} 


\subsection{Growth condition}

Since we take $X_{R}=B_{\mathcal{H}^{r}}(R)$, the set $X_{\infty}$ in the growth condition in Theorem A.4 will be equal to $\mathcal{H}^{r}$ which is dense in $\mathcal{H}$. For any $\mathfrak{u} \in \mathcal{H}^{r}$ and $t \geq 0$, we have $\mathfrak{u}(t ; \mathfrak{u}) \in \mathcal{H}^{r}$, so the measure $P_{t}^{V}(\mathfrak{u}, \cdot)$ is concentrated on $\mathcal{H}^{r}$. As $V$ is a bounded function, condition (A.12) is verified. Let us show that estimate (A.11) holds for any $V$ with a sufficiently small oscillation.

Proposition 3.1. There is a constant $\delta>0$ and an integer $R_{0} \geq 1$ such that, for any $V \in C_{b}(\mathcal{H})$ satisfying $\operatorname{Osc}(V)<\delta$, we have

$$
\sup _{t \geq 0} \frac{\left\|\mathfrak{P}_{t}^{V} \mathfrak{w}\right\|_{L_{\mathfrak{w}}^{\infty}}}{\left\|\mathfrak{P}_{t}^{V} \mathbf{1}\right\|_{R_{0}}}<\infty,
$$

where 1 is the function on $\mathcal{H}$ identically equal to 1 and $\|\cdot\|_{R_{0}}$ is the $L^{\infty}$ norm on $X_{R_{0}}$.

Proof. Without loss of generality, we can assume that $V \geq 0$ and $\operatorname{Osc}(V)=\|V\|_{\infty}$. Indeed, it suffices to replace $V$ by $V-\inf _{H} V$. We split the proof of (3.1) into two steps.

Step 1. Let us show that there are $\delta_{0}>0$ and $R_{0} \geq 1$ such that

$$
\sup _{t \geq 0} \frac{\left\|\mathfrak{P}_{t}^{V} \mathbf{1}\right\|_{L_{\mathfrak{w}}^{\infty}}}{\left\|\mathfrak{P}_{t}^{V} \mathbf{1}\right\|_{R_{0}}}<\infty,
$$

provided that $\|V\|_{\infty}<\delta_{0}$. To prove this, we introduce the stopping time

$$
\tau(R)=\inf \left\{t \geq 0:\left|\mathfrak{u}_{t}\right| \mathcal{H}^{r} \leq R\right\}
$$

and use the following result.

Lemma 3.2. There are positive numbers $\delta_{0}, C$, and $R_{0}$ such that

$$
\mathbb{E}_{\mathfrak{u}} e^{\delta_{0} \tau\left(R_{0}\right)} \leq C \mathfrak{w}(\mathfrak{u}), \quad \mathfrak{u} \in \mathcal{H}^{r} .
$$

We omit the proof of this lemma, since it is carried out by standard arguments, using the Lyapunov function $\mathfrak{w}$ and estimate (5.24) for $m=1$ (see Lemma 3.6.1 in [19]). Setting $G_{t}:=\left\{\tau\left(R_{0}\right)>t\right\}$ and

$$
\Xi_{V}(t):=\exp \left(\int_{0}^{t} V\left(\mathfrak{u}_{s}\right) \mathrm{d} s\right)
$$

we get

$$
\mathfrak{P}_{t}^{V} \mathbf{1}(\mathfrak{u})=\mathbb{E}_{\mathfrak{u}} \Xi_{V}(t)=\mathbb{E}_{\mathfrak{u}}\left\{\mathbb{I}_{G_{t}} \Xi_{V}(t)\right\}+\mathbb{E}_{\mathfrak{u}}\left\{\mathbb{I}_{G_{t}^{c}} \Xi_{V}(t)\right\}=: I_{1}+I_{2} .
$$

Since $V \geq 0$, we have $\mathfrak{P}_{t}^{V} \mathbf{1}(\mathfrak{u}) \geq 1$. Combining this with (3.3) and $\|V\|_{\infty}<\delta_{0}$, we obtain for any $\mathfrak{u} \in \mathcal{H}^{r}$

$$
I_{1} \leq \mathbb{E}_{\mathfrak{u}} \Xi_{V}\left(\tau\left(R_{0}\right)\right) \leq \mathbb{E}_{\mathfrak{u}} \exp \left(\delta_{0} \tau\left(R_{0}\right)\right) \leq C \mathfrak{w}(\mathfrak{u}) \leq C \mathfrak{w}(\mathfrak{u})\left\|\mathfrak{P}_{t}^{V} \mathbf{1}\right\|_{R_{0}}
$$

The strong Markov property and (3.3) imply

$$
\begin{aligned}
I_{2} & \leq \mathbb{E}_{\mathfrak{u}}\left\{\mathbb{I}_{G_{t}} \Xi_{V}\left(\tau\left(R_{0}\right)\right) \mathbb{E}_{\mathfrak{u}\left(\tau\left(R_{0}\right)\right)} \Xi_{V}(t)\right\} \\
& \leq \mathbb{E}_{\mathfrak{u}}\left\{e^{\delta_{0} \tau\left(R_{0}\right)}\right\}\left\|\mathfrak{P}_{t}^{V} \mathbf{1}\right\|_{R_{0}} \leq C \mathfrak{w}(\mathfrak{u})\left\|\mathfrak{P}_{t}^{V} \mathbf{1}\right\|_{R_{0}},
\end{aligned}
$$

where we write $\mathfrak{u}\left(\tau\left(R_{0}\right)\right)$ instead of $\mathfrak{u}_{\tau\left(R_{0}\right)}$. Using (3.5) and the estimates for $I_{1}$ and $I_{2}$, we get (3.2). 
Step 2. To prove (3.1), we set $\delta:=\delta_{0} \wedge(\alpha / 2)$ and assume that $\|V\|_{\infty}<\delta$ and $t=T k$, where $k \geq 1$ is an integer and $T>0$ is so large that $q:=2 e^{-T \frac{\alpha}{2}}<1$. Then, using the Markov property and (5.24), we get

$$
\begin{aligned}
\mathfrak{P}_{T k}^{V} \mathfrak{w}(\mathfrak{u}) & \leq e^{T \delta} \mathbb{E}_{\mathfrak{u}}\left\{\Xi_{V}(T(k-1)) \mathfrak{w}\left(\mathfrak{u}_{T k}\right)\right\} \\
& =e^{T \delta} \mathbb{E}_{\mathfrak{u}}\left\{\Xi_{V}(T(k-1)) \mathbb{E}_{\mathfrak{u}(T(k-1))} \mathfrak{w}\left(\mathfrak{u}_{T}\right)\right\} \\
& \leq e^{T \delta} \mathbb{E}_{\mathfrak{u}}\left\{\Xi_{V}(T(k-1))\left[2 e^{-T \alpha} \mathfrak{w}\left(\mathfrak{u}_{T(k-1)}\right)+C_{1}\right]\right\} \\
& \leq q \mathfrak{P}_{T(k-1)}^{V} \mathfrak{w}(\mathfrak{u})+e^{T \delta} C_{1} \mathfrak{P}_{T(k-1)}^{V} \mathbf{1}(\mathfrak{u}) .
\end{aligned}
$$

Iterating this and using fact that $V \geq 0$, we obtain

$$
\mathfrak{P}_{T k}^{V} \mathfrak{w}(\mathfrak{u}) \leq q^{k} \mathfrak{w}(\mathfrak{u})+(1-q)^{-1} e^{T \delta} C_{1} \mathfrak{P}_{T k}^{V} \mathbf{1}(\mathfrak{u}) .
$$

Combining this with (3.2), we see that

$$
A:=\sup _{k \geq 0} \frac{\left\|\mathfrak{P}_{T k}^{V} \mathfrak{w}\right\|_{L_{\mathfrak{w}}^{\infty}}}{\left\|\mathfrak{P}_{T k}^{V} \mathbf{1}\right\|_{R_{0}}}<\infty .
$$

To derive (3.1) from this, we use the semigroup property and the fact that $V$ is non-negative and bounded:

$$
\begin{aligned}
& \left\|\mathfrak{P}_{t}^{V} \mathfrak{w}\right\|_{L_{\mathfrak{w}}^{\infty}}=\left\|\mathfrak{P}_{t-T k}^{V}\left(\mathfrak{P}_{T k}^{V} \mathfrak{w}\right)\right\|_{L_{\mathfrak{w}}^{\infty}} \leq C_{2}\left\|\mathfrak{P}_{T k}^{V} \mathfrak{w}\right\|_{L_{\mathfrak{w}}^{\infty}}, \\
& \left\|\mathfrak{P}_{t}^{V} \mathbf{1}\right\|_{R_{0}} \geq\left\|\mathfrak{P}_{T k}^{V} \mathbf{1}\right\|_{R_{0}},
\end{aligned}
$$

where $k \geq 0$ is such that $T k \leq t<T(k+1)$ and

$$
C_{2}:=\sup _{s \in[0, T]}\left\|\mathfrak{P}_{s}^{V} \mathfrak{w}\right\|_{L_{\mathfrak{w}}^{\infty}} \leq e^{T\|V\|_{\infty}} \sup _{s \in[0, T]}\left\|\mathfrak{P}_{s} \mathfrak{w}\right\|_{L_{\mathfrak{w}}^{\infty}}<\infty
$$

So we get

$$
\sup _{t \geq 0} \frac{\left\|\mathfrak{P}_{t}^{V} \mathfrak{w}\right\|_{L_{\mathfrak{w}}^{\infty}}}{\left\|\mathfrak{P}_{t}^{V} \mathbf{1}\right\|_{R_{0}}} \leq C_{2} A<+\infty .
$$

This completes the proof of the proposition.

\subsection{Uniform irreducibility}

In this section, we show that the family $\left\{P_{t}^{V}\right\}$ satisfies the uniform irreducibility condition with respect to the sequence of compacts $\left\{X_{R}\right\}$. Since $V$ is bounded, we have

$$
P_{t}^{V}(\mathfrak{u}, \mathrm{d} \mathfrak{v}) \geq e^{-t\|V\|_{\infty}} P_{t}(\mathfrak{u}, \mathrm{d} \mathfrak{v}), \quad \mathfrak{u} \in \mathcal{H},
$$

where $P_{t}(\mathfrak{u}, \cdot)$ stands for the transition function of $\left(\mathfrak{u}_{t}, \mathbb{P}_{\mathfrak{u}}\right)$. So it suffices to establish the uniform irreducibility for $\left\{P_{t}\right\}$.

Proposition 3.3. For any integers $\rho, R \geq 1$ and any $r>0$, there are positive numbers $l=l(\rho, r, R)$ and $p=p(\rho, r)$ such that

$$
P_{l}\left(\mathfrak{u}, B_{\mathcal{H}}(\hat{\mathfrak{u}}, r)\right) \geq p \quad \text { for all } \mathfrak{u} \in X_{R}, \hat{\mathfrak{u}} \in X_{\rho} .
$$

Proof. Let us show that, for sufficiently large $d \geq 1$ and any $R \geq 1$, there is a time $k=k(R)$ such that

$$
P_{k}\left(\mathfrak{u}, X_{d}\right) \geq \frac{1}{2}, \quad \mathfrak{u} \in X_{R}
$$


Indeed, by (5.24) for $m=1$, we have

$$
\mathbb{E}_{\mathfrak{u}}\left|\mathfrak{u}_{t}\right|_{\mathcal{H}^{r}}^{2} \leq \mathbb{E}_{\mathfrak{u}} \mathfrak{w}\left(\mathfrak{u}_{t}\right) \leq 2 e^{-\alpha t} \mathfrak{w}(\mathfrak{u})+C_{1} .
$$

Combining this with the estimate

$$
|\mathcal{E}(\mathfrak{u})| \leq C_{2}\left(1+|\mathfrak{u}|_{\mathcal{H}}^{4}\right),
$$

we get

$$
\mathbb{E}_{\mathfrak{u}}\left|\mathfrak{u}_{t}\right|_{\mathcal{H}^{r}}^{2} \leq C_{3} e^{-\alpha t} R^{16}+C_{1}, \quad \mathfrak{u} \in X_{R}
$$

The Chebyshev inequality implies that

$$
P_{t}\left(\mathfrak{u}, X_{d}\right) \geq 1-d^{-2}\left(C_{3} e^{-\alpha t} R^{16}+C_{1}\right) .
$$

Choosing $t=k$ and $d$ so large that $e^{-\alpha k} R^{16} \leq 1$ and $d^{2}>2\left(C_{3}+C_{1}\right)$, we obtain (3.7).

Combining (3.7) with Lemma 3.4 and the Kolmogorov-Chapman relation, we get (3.6) for $l=k+m$ and $p=$ $q / 2$.

Lemma 3.4. For any integers $d, \rho \geq 1$ and any $r>0$, there are positive numbers $m=m(d, \rho, r)$ and $q=q(d, \rho, r)$ such that

$$
P_{m}\left(\mathfrak{v}, B_{\mathcal{H}}(\hat{\mathfrak{u}}, r)\right) \geq q \quad \text { for all } \mathfrak{v} \in X_{d}, \hat{\mathfrak{u}} \in X_{\rho} .
$$

Proof. It is sufficient to prove that there is $m \geq 1$ such that

$$
P_{m}\left(\mathfrak{v}, B_{\mathcal{H}}(\hat{\mathfrak{u}}, r / 2)\right)>0 \quad \text { for all } \mathfrak{v} \in X_{d}, \hat{\mathfrak{u}} \in \tilde{X}_{\rho},
$$

where $\tilde{X}_{\rho}=\left\{\mathfrak{u}=\left[u_{1}, u_{2}\right] \in X_{\rho}: u_{1}, u_{2} \in C_{0}^{\infty}(D)\right\}$. Indeed, let us take this inequality for granted and assume that (3.9) is not true. Then there are sequences $\mathfrak{v}_{j} \in X_{d}$ and $\hat{\mathfrak{u}}_{j} \in X_{\rho}$ such that

$$
P_{m}\left(\mathfrak{v}_{j}, B_{\mathcal{H}}\left(\hat{\mathfrak{u}}_{j}, r\right)\right) \rightarrow 0
$$

Moreover, up to extracting a subsequence, we can suppose that $\mathfrak{v}_{j}$ and $\hat{\mathfrak{u}}_{j}$ converge in $\mathcal{H}$. Let us denote by $\mathfrak{v}_{*}$ and $\hat{\mathfrak{u}}_{*}$ their limits. Clearly, $\mathfrak{v}_{*} \in X_{d}$ and $\hat{\mathfrak{u}}_{*} \in X_{\rho}$. Choosing $j \geq 1$ so large that $\left|\hat{\mathfrak{u}}_{j}-\hat{\mathfrak{u}}_{*}\right| \mathcal{H}<r / 2$ and applying the Chebyshev inequality, we get

$$
\begin{aligned}
P_{m}\left(\mathfrak{v}_{*}, B_{\mathcal{H}}\left(\hat{\mathfrak{u}}_{*}, r\right)\right) & \leq P_{m}\left(\mathfrak{v}_{j}, B_{\mathcal{H}}\left(\hat{\mathfrak{u}}_{j}, r / 2\right)\right)+\mathbb{P}\left\{\left|\mathfrak{u}\left(m ; \mathfrak{v}_{j}\right)-\mathfrak{u}\left(m ; \mathfrak{v}_{*}\right)\right|_{\mathcal{H}} \geq r / 2\right\} \\
& \leq P_{m}\left(\mathfrak{v}_{j}, B_{\mathcal{H}}\left(\hat{\mathfrak{u}}_{j}, r / 2\right)\right)+4 / r^{2} \mathbb{E}\left|\mathfrak{u}\left(m ; \mathfrak{v}_{j}\right)-\mathfrak{u}\left(m ; \mathfrak{v}_{*}\right)\right|_{\mathcal{H}}^{2}
\end{aligned}
$$

Combining this with (3.11) and using the convergence $\mathfrak{v}_{j} \rightarrow \mathfrak{v}_{*}$ and a density property, we arrive at a contradiction with (3.10). Thus, inequality (3.9) is reduced to the derivation of (3.10). We shall prove the latter in three steps.

Step 1: Exact controllability. In what follows, given any $\varphi \in C\left(0, T ; H^{1}\right)$, we shall denote by $S_{\varphi}(t ; \mathfrak{v})$ the solution at time $t$ of the problem

$$
\partial_{t}^{2} u+\gamma \partial_{t} u-\Delta u+f(u)=h+\dot{\varphi},\left.\quad u\right|_{\partial D}=0, t \in[0, T]
$$

issued from $\mathfrak{v}$. Let $\hat{\mathfrak{v}}=[\hat{v}, 0]$, where $\hat{v} \in H^{1}$ is a solution of

$$
-\Delta \hat{v}+f(\hat{v})=h .
$$

In this step we prove that for any $\hat{\mathfrak{u}}=\left[\hat{u}_{1}, \hat{u}_{2}\right] \in \tilde{X}_{\rho}$, there is $\varphi_{*}$ satisfying

$$
\varphi_{*} \in C\left(0,1 ; H^{1}\right) \quad \text { and } \quad S_{\varphi_{*}}(1 ; \hat{\mathfrak{v}})=\hat{\mathfrak{u}} .
$$


First note that, since the function $f$ is continuous from $H^{1}$ to $L^{2}$, we have

$$
-\Delta \hat{v}=-f(\hat{v})+h \in L^{2},
$$

so that $\hat{v} \in H^{2}$. Moreover, since $f$ is also continuous from $H^{2}$ to $H^{1}$ (recall that $f$ vanishes at the origin), we have $f(\hat{v}) \in H^{1}$. As $h \in H^{1}$, it follows that

$$
-\Delta \hat{v} \in H^{1}
$$

Let us introduce the functions

$$
\begin{aligned}
& u(t)=a(t) \hat{v}+b(t) \hat{u}_{1}+c(t) \hat{u}_{2}, \\
& \varphi_{*}(t)=\int_{0}^{t}\left(\partial_{t}^{2} u+\gamma \partial_{t} u-\Delta u+f(u)-h\right) \mathrm{d} \tau,
\end{aligned}
$$

where $a, b, c \in C^{\infty}([0,1], \mathbb{R})$ satisfy

$$
\begin{array}{ll}
a(0)=1, & a(1)=\dot{a}(0)=\dot{a}(1)=0, \quad b(1)=1, \quad b(0)=\dot{b}(0)=\dot{b}(1)=0, \\
\dot{c}(1)=1, & c(0)=c(1)=\dot{c}(0)=0 .
\end{array}
$$

Then, we have $[u(0), \dot{u}(0)]=\hat{\mathfrak{v}},[u(1), \dot{u}(1)]=\hat{\mathfrak{u}}$, and $S_{\varphi_{*}}(1 ; \hat{\mathfrak{v}})=\hat{\mathfrak{u}}$. Let us show the first relation in (3.12). In view of (3.14) and the smoothness of the functions $a, b$ and $c$, we have

$$
\partial_{t}^{2} u+\gamma \partial_{t} u-h \in C\left(0,1 ; H^{1}\right)
$$

and thus it is sufficient to prove that

$$
-\Delta u+f(u) \in C\left(0,1 ; H^{1}\right) .
$$

Since $u \in C\left(0,1 ; H^{2}\right)$, we have $f(u) \in C\left(0,1 ; H^{1}\right)$. Moreover, in view of (3.13) and the smoothness of $\hat{u}_{1}$ and $\hat{u}_{2}$, we have $-\Delta u \in C\left(0,1 ; H^{1}\right)$. Thus, inclusion (3.15) is established and we arrive at (3.12). Let us note that by continuity and compactness, there is $\varkappa=\varkappa(\hat{\mathfrak{v}}, \rho, r)>0$, not depending on $\hat{\mathfrak{u}} \in \tilde{X}_{\rho}$, such that

$$
S_{\varphi_{*}}(1 ; \mathfrak{v}) \in B_{\mathcal{H}}(\hat{\mathfrak{u}}, r / 4) \quad \text { for any } \mathfrak{v} \in B_{\mathcal{H}}(\hat{\mathfrak{v}}, \varkappa) .
$$

Step 2: Feedback stabilization. We now show that there is $\tilde{m} \geq 1$ depending only on $d$ and $\varkappa$ such that for any $\mathfrak{v} \in X_{d}$ there is $\tilde{\varphi}_{\mathfrak{v}}$ satisfying

$$
\tilde{\varphi}_{\mathfrak{v}} \in C\left(0, \tilde{m} ; H^{1}\right) \quad \text { and } \quad S_{\tilde{\varphi}_{\mathfrak{v}}}(\tilde{m}, \mathfrak{v}) \in B(\hat{\mathfrak{v}}, \varkappa) .
$$

To see this, let us consider the flow $\tilde{\mathfrak{v}}(t ; \mathfrak{v})$ associated with the solution of the equation

$$
\partial_{t}^{2} \tilde{v}+\gamma \partial_{t} \tilde{v}-\Delta \tilde{v}+f(\tilde{v})=h+\mathrm{P}_{N}[f(\tilde{v})-f(\hat{v})], \quad t \in[0, \tilde{m}]
$$

issued from $\mathfrak{v} \in X_{d}$, where $\mathrm{P}_{N}$ stands for the orthogonal projection in $L^{2}$ onto the subspace spanned by the functions $e_{1}, e_{2}, \ldots, e_{N}$. Then, in view of Proposition 6.5 in [22], for $N \geq N\left(|\hat{\mathfrak{v}}|_{\mathcal{H}}, d\right)$, we have

$$
|\tilde{\mathfrak{v}}(\tilde{m} ; \mathfrak{v})-\hat{\mathfrak{v}}|_{\mathcal{H}}^{2} \leq|\mathfrak{v}-\hat{\mathfrak{v}}|_{\mathcal{H}}^{2} e^{-\alpha \tilde{m}} \leq C_{d} e^{-\alpha \tilde{m}}<\varkappa
$$

for $\tilde{m}$ sufficiently large. It follows that (3.17) holds with the function

$$
\tilde{\varphi}_{\mathfrak{v}}(t)=\int_{0}^{t} \mathrm{P}_{N}[f(\tilde{v})-f(\hat{v})] \mathrm{d} \tau .
$$


Step 3: Proof of (3.10). Let us take $m=\tilde{m}+1$ and, for any $\mathfrak{v} \in X_{d}$, define a function $\varphi_{\mathfrak{v}}(t)$ on the interval $[0, m]$ by

$$
\varphi_{\mathfrak{v}}(t)= \begin{cases}\tilde{\varphi}_{\mathfrak{v}}(t) & \text { for } t \in[0, m-1], \\ \tilde{\varphi}_{\mathfrak{v}}(m-1)+\varphi_{*}(t-m+1) & \text { for } t \in[m-1, m] .\end{cases}
$$

In view of (3.12), (3.16), and (3.17), we have $\varphi_{\mathfrak{v}}(t) \in C\left(0, m ; H^{1}\right)$ and $S_{\varphi_{\mathfrak{v}}}(m ; \mathfrak{v}) \in B_{\mathcal{H}}(\hat{\mathfrak{u}}, r / 2)$. Hence there is $\delta>0$ such that $S_{\varphi}(m ; \mathfrak{v}) \in B_{\mathcal{H}}(\hat{\mathfrak{u}}, r / 2)$ provided $\left\|\varphi-\varphi_{\mathfrak{v}}\right\|_{C\left(0, m ; H^{1}\right)}<\delta$. It follows that

$$
P_{m}\left(\mathfrak{v}, B_{\mathcal{H}}(\hat{\mathfrak{u}}, r / 2)\right) \geq \mathbb{P}\left\{\left\|\xi-\varphi_{\mathfrak{v}}\right\|_{C\left(0, m ; H^{1}\right)}<\delta\right\} .
$$

To complete the proof, it remains to note that, due to the non-degeneracy of $\xi$, the term on the right-hand side of this inequality is positive.

\subsection{Existence of an eigenvector}

For any $m \geq 1$, let us define functions $\mathfrak{w}_{m}, \tilde{\mathfrak{w}}_{m}: \mathcal{H} \rightarrow[1,+\infty]$ by

$$
\begin{aligned}
& \mathfrak{w}_{m}(\mathfrak{u})=1+|\mathfrak{u}|_{\mathcal{H}^{r}}^{2 m}+\mathcal{E}^{4 m}(\mathfrak{u}), \\
& \tilde{\mathfrak{w}}_{m}(\mathfrak{u})=\mathfrak{w}_{m}(\mathfrak{u})+\exp (\varkappa \mathcal{E}(\mathfrak{u})), \quad \mathfrak{u} \in \mathcal{H},
\end{aligned}
$$

where $\varkappa:=(2 \alpha)^{-1} B_{0}$ and $B_{0}:=\sum b_{j}^{2}$. The following proposition proves the existence of an eigenvector $\mu=$ $\mu(t, V, m)$ for the operator $\mathfrak{P}_{t}^{V *}$ for any $t>0$. We shall see in Section 6 that the measure $\mu$ actually does not depend on $t$ and $m$.

Proposition 3.5. For any $t>0, V \in C_{b}(\mathcal{H})$ and $m \geq 1$, the operator $\mathfrak{P}_{t}^{V *}$ admits an eigenvector $\mu=\mu(t, V, m) \in$ $\mathcal{P}(\mathcal{H})$ with a positive eigenvalue $\lambda=\lambda(t, V, m)$ :

$$
\mathfrak{P}_{t}^{V *} \mu=\lambda \mu \text {. }
$$

Moreover, we have

$$
\begin{aligned}
& \int_{\mathcal{H}} \tilde{\mathfrak{w}}_{m}(\mathfrak{u}) \mu(\mathrm{d} \mathfrak{u})<\infty \\
& \left\|\mathfrak{P}_{t}^{V} \mathfrak{w}_{m}\right\|_{X_{R}} \int_{X_{R}^{c}} \mathfrak{w}_{m}(\mathfrak{u}) \mu(\mathrm{d} \mathfrak{u}) \rightarrow 0 \quad \text { as } R \rightarrow \infty .
\end{aligned}
$$

Proof. Step 1. We first establish the existence of an eigenvector $\mu$ for $\mathfrak{P}_{t}^{V *}$ with a positive eigenvalue and satisfying (3.21). Let $t>0$ and $V$ be fixed. For any $A>0$ and $m \geq 1$, let us introduce the convex set

$$
D_{A, m}=\left\{\sigma \in \mathcal{P}(\mathcal{H}):\left\langle\tilde{\mathfrak{w}}_{m}, \sigma\right\rangle \leq A\right\},
$$

and consider the continuous mapping from $D_{A, m}$ to $\mathcal{P}(\mathcal{H})$ given by

$$
G(\sigma)=\mathfrak{P}_{t}^{V *} \sigma / \mathfrak{P}_{t}^{V *} \sigma(\mathcal{H}) .
$$

Thanks to inequality (5.25), we have

$$
\begin{aligned}
\left\langle\tilde{\mathfrak{w}}_{m}, G(\sigma)\right\rangle & \leq \exp \left(t \operatorname{Osc}_{\mathcal{H}}(V)\right)\left\langle\tilde{\mathfrak{w}}_{m}, \mathfrak{P}_{t}^{*} \sigma\right\rangle \\
& \leq 2 \exp \left(t\left(\operatorname{Osc}_{\mathcal{H}}(V)-\alpha m\right)\right)\left\langle\tilde{\mathfrak{w}}_{m}, \sigma\right\rangle+C_{m} \exp \left(t \operatorname{Osc}_{\mathcal{H}}(V)\right) .
\end{aligned}
$$


Assume that $m$ is so large that

$$
\operatorname{Osc}_{\mathcal{H}}(V) \leq \alpha m / 2 \quad \text { and } \quad \exp (-\alpha m t / 2) \leq 1 / 4,
$$

and let $A:=2 C_{m} e^{\alpha m t}$. Then, in view (3.23), we have $\left\langle\tilde{\mathfrak{w}}_{m}, G(\sigma)\right\rangle \leq A$ for any $\sigma \in D_{A, m}$, i.e., $G\left(D_{A, m}\right) \subset D_{A, m}$. Moreover, it is easy to see that the set $D_{A, m}$ is compact in $\mathcal{P}(\mathcal{H})$ (we use the Prokhorov compactness criterion to show that it is relatively compact and the Fatou lemma to prove that it is closed). Due to the Leray-Schauder theorem, the map $G$ has a fixed point $\mu \in D_{A, m}$. Note that, by the definitions of $D_{A, m}$ and $G$, the measure $\mu$ is an eigenvector of $\mathfrak{P}_{t}^{V *}$ with positive eigenvalue $\lambda:=\mathfrak{P}_{t}^{V *} \mu(\mathcal{H})$ and satisfies (3.21).

Step 2. We now establish (3.22). Let us fix an integer $m \geq 1$ and let $n=17 \mathrm{~m}$. In view of the previous step, there is an eigenvector $\mu$ satisfying $\left\langle\mathfrak{w}_{n}, \mu\right\rangle<\infty$. From the Cauchy-Schwarz and Chebyshev inequalities it follows that

$$
\int_{X_{R}^{c}} \mathfrak{w}_{m}(\mathfrak{u}) \mu(\mathrm{d} \mathfrak{u}) \leq\left\langle\mathfrak{w}_{m}^{2}, \mu\right\rangle^{1 / 2}\left(\mu\left(X_{R}^{c}\right)\right)^{1 / 2} \leq C_{m}\left\langle\mathfrak{w}_{n}, \mu_{t, V}\right\rangle R^{-n} .
$$

On the other hand, using (5.24) and (3.8), we get

$$
\left\|\mathfrak{P}_{t}^{V} \mathfrak{w}_{m}\right\|_{X_{R}} \leq \exp \left(t\|V\|_{\infty}\right) \sup _{\mathfrak{u} \in X_{R}} \mathbb{E}_{\mathfrak{u}} \mathfrak{w}_{m}\left(\mathfrak{u}_{t}\right) \leq C_{m}^{\prime} \exp \left(t\|V\|_{\infty}\right)\left(R^{16 m}+1\right)
$$

Combining this with (3.24), we obtain (3.22).

\section{Uniform Feller property}

\subsection{Construction of coupling processes}

As in the case of discrete-time models considered in [10,12], the proof of the uniform Feller property is based on the coupling method. This method has proved to be an important tool for the study of the ergodicity of randomly forced PDE's (see Chapter 3 in [19] and the papers [18,21,23,24]). In this section, we recall a construction of coupled trajectories from [21], which was used to establish the exponential mixing for problem $(0.1),(0.3)$. This construction will play a central role in the proof of the uniform Feller property in the next section.

For any $\mathfrak{z}, \mathfrak{z}^{\prime} \in \mathcal{H}$, let us denote by $\mathfrak{u}_{t}$ and $\mathfrak{u}_{t}^{\prime}$ the flows of $(0.1),(0.3)$ issued from $\mathfrak{z}$ and $\mathfrak{z}^{\prime}$, respectively. As above, $\mathrm{P}_{N}$ is the orthogonal projection in $L^{2}$ onto the space $\operatorname{span}\left\{e_{1}, \ldots, e_{N}\right\}$. Let us take any integer $N \geq 1$ and consider the flow $\mathfrak{v}=\left[v, \partial_{t} v\right]$ of the problem

$$
\partial_{t}^{2} v+\gamma \partial_{t} v-\Delta v+f(v)+\mathrm{P}_{N}(f(u)-f(v))=h+\vartheta(t, x),\left.\quad v\right|_{\partial D}=0, \mathfrak{v}(0)=\mathfrak{z}^{\prime} .
$$

The laws of the processes $\left\{\mathfrak{v}_{t}, t \in[0,1]\right\}$ and $\left\{\mathfrak{u}_{t}^{\prime}, t \in[0,1]\right\}$ are denoted by $\lambda\left(\mathfrak{z}, \mathfrak{z}^{\prime}\right)$ and $\lambda\left(\mathfrak{z}^{\prime}\right)$, respectively. We have the following estimate for the total variation distance between $\lambda\left(\mathfrak{z}, \mathfrak{z}^{\prime}\right)$ and $\lambda\left(\mathfrak{z}^{\prime}\right)$.

Proposition 4.1. There is an integer $N_{1} \geq 1$ such that, for any $N \geq N_{1}, \varepsilon>0$, and $\mathfrak{z}, \mathfrak{z}^{\prime} \in \mathcal{H}$, we have

$$
\left|\lambda\left(\mathfrak{z}, \mathfrak{z}^{\prime}\right)-\lambda\left(\mathfrak{z}^{\prime}\right)\right|_{\mathrm{var}} \leq C_{*} \varepsilon^{a}+C_{*}\left[\exp \left(C_{N} \varepsilon^{a-2}\left|\mathfrak{z}-\mathfrak{z}^{\prime}\right|_{\mathcal{H}}^{2} e^{\left(|\mathcal{E}(\mathfrak{z})|+\left|\mathcal{E}\left(\mathfrak{z}^{\prime}\right)\right|\right)}\right)-1\right]^{1 / 2},
$$

where $a<2, C_{*}$, and $C_{N}$ are positive numbers not depending on $\varepsilon, \mathfrak{z}$, and $\mathfrak{z}^{\prime}$.

See Section A.3 for the proof of this proposition. By Proposition 1.2.28 in [19], there is a probability space $(\hat{\Omega}, \hat{\mathcal{F}}, \hat{\mathbb{P}})$ and measurable functions $\mathcal{Z}, \mathcal{Z}^{\prime}: \mathcal{H} \times \mathcal{H} \times \hat{\Omega} \rightarrow C([0,1], \mathcal{H})$ such that $\left(\mathcal{Z}\left(\mathfrak{z}, \mathfrak{z}^{\prime}\right), \mathcal{Z}^{\prime}\left(\mathfrak{z}, \mathfrak{z}^{\prime}\right)\right)$ is a maximal coupling for $\left(\lambda\left(\mathfrak{z}, \mathfrak{z}^{\prime}\right), \lambda\left(\mathfrak{z}^{\prime}\right)\right)$ for any $\mathfrak{z}, \mathfrak{z}^{\prime} \in \mathcal{H}$. We denote by $\tilde{\mathfrak{v}}=\left[\tilde{v}_{t}, \partial_{t} \tilde{v}\right]$ and $\tilde{\mathfrak{u}}_{t}^{\prime}=\left[\tilde{u}_{t}^{\prime}, \partial_{t} \tilde{u}^{\prime}\right]$ the restrictions of $\mathcal{Z}$ and $\mathcal{Z}^{\prime}$ to time $t \in[0,1]$. Then $\tilde{v}_{t}$ is a solution of the problem

$$
\partial_{t}^{2} \tilde{v}+\gamma \partial_{t} \tilde{v}-\Delta \tilde{v}+f(\tilde{v})-\mathrm{P}_{N} f(\tilde{v})=h+\psi(t),\left.\quad \tilde{v}\right|_{\partial D}=0, \tilde{\mathfrak{v}}(0)=\mathfrak{z}^{\prime},
$$


where the process $\left\{\int_{0}^{t} \psi(\tau) \mathrm{d} \tau, t \in[0,1]\right\}$ has the same law as

$$
\left\{\xi(t)-\int_{0}^{t} \mathrm{P}_{N} f\left(u_{\tau}\right) \mathrm{d} \tau, t \in[0,1]\right\} .
$$

Let $\tilde{\mathfrak{u}}_{t}=\left[\tilde{u}, \partial_{t} \tilde{u}\right]$ be a solution of

$$
\partial_{t}^{2} \tilde{u}+\gamma \partial_{t} \tilde{u}-\Delta \tilde{u}+f(\tilde{u})-\mathrm{P}_{N} f(\tilde{u})=h+\psi(t),\left.\quad \tilde{u}\right|_{\partial D}=0, \tilde{\mathfrak{u}}(0)=\mathfrak{z} .
$$

Then $\left\{\tilde{\mathfrak{u}}_{t}, t \in[0,1]\right\}$ has the same law as $\left\{\mathfrak{u}_{t}, t \in[0,1]\right\}$ (see Section 6.1 in [21] for the proof). Now the coupling operators $\mathcal{R}$ and $\mathcal{R}^{\prime}$ are defined by

$$
\mathcal{R}_{t}\left(\mathfrak{z}, \mathfrak{z}^{\prime}, \omega\right)=\tilde{\mathfrak{u}}_{t}, \quad \mathcal{R}_{t}^{\prime}\left(\mathfrak{z}, \mathfrak{z}^{\prime}, \omega\right)=\tilde{\mathfrak{u}}_{t}^{\prime}, \quad \mathfrak{z}, \mathfrak{z}^{\prime} \in \mathcal{H}, \omega \in \hat{\Omega}
$$

By Proposition 4.1 , if $N \geq N_{1}$, then for any $\varepsilon>0$, we have

$$
\begin{aligned}
& \hat{\mathbb{P}}\left\{\exists t \in[0,1] \text { s.t. } \tilde{\mathfrak{v}}_{t} \neq \tilde{\mathfrak{u}}_{t}^{\prime}\right\} \\
& \quad \leq C_{*} \varepsilon^{a}+C_{*}\left[\exp \left(C_{N} \varepsilon^{a-2}\left|\mathfrak{z}-\mathfrak{z}^{\prime}\right|_{\mathcal{H}}^{2} e^{\left(|\mathcal{E}(\mathfrak{z})|+\left|\mathcal{E}\left(\mathfrak{z}^{\prime}\right)\right|\right)}\right)-1\right]^{1 / 2} .
\end{aligned}
$$

Let $\left(\Omega^{k}, \mathcal{F}^{k}, \mathbb{P}^{k}\right), k \geq 0$ be a sequence of independent copies of the probability space $(\hat{\Omega}, \hat{\mathcal{F}}, \hat{\mathbb{P}})$. We denote by $(\Omega, \mathcal{F}, \mathbb{P})$ the direct product of the spaces $\left(\Omega^{k}, \mathcal{F}^{k}, \mathbb{P}^{k}\right)$, and for any $\mathfrak{z}, \mathfrak{z}^{\prime} \in \mathcal{H}, \omega=\left(\omega^{1}, \omega^{2}, \ldots\right) \in \Omega$, and $k \geq 0$, we set $\tilde{u}_{0}=u, \tilde{u}_{0}^{\prime}=u^{\prime}$, and

$$
\begin{aligned}
& \tilde{\mathfrak{u}}_{t}(\omega)=\mathcal{R}_{\tau}\left(\tilde{\mathfrak{u}}_{k}(\omega), \tilde{\mathfrak{u}}_{k}^{\prime}(\omega), \omega^{k}\right), \quad \tilde{\mathfrak{u}}_{t}^{\prime}(\omega)=\mathcal{R}_{\tau}^{\prime}\left(\tilde{\mathfrak{u}}_{k}(\omega), \tilde{\mathfrak{u}}_{k}^{\prime}(\omega), \omega^{k}\right), \\
& \tilde{\mathfrak{v}}_{t}(\omega)=\mathcal{Z}_{\tau}\left(\tilde{\mathfrak{u}}_{k}(\omega), \tilde{\mathfrak{u}}_{k}^{\prime}(\omega), \omega^{k}\right),
\end{aligned}
$$

where $t=\tau+k, \tau \in[0,1)$. We shall say that $\left(\tilde{\mathfrak{u}}_{t}, \tilde{\mathfrak{u}}_{t}^{\prime}\right)$ is a coupled trajectory at level $N$ issued from $\left(\mathfrak{z}, \mathfrak{z}^{\prime}\right)$.

\subsection{The result and its proof}

The following theorem establishes the uniform Feller property for the semigroup $\mathfrak{P}_{t}^{V}$ for any function $V \in \mathcal{U}_{\delta}$ with sufficiently small $\delta>0$. The property is proved with respect to the space $\mathcal{C}=\mathcal{U}$ which is a determining family for $\mathcal{P}(\mathcal{H})$ and contains the constant functions.

Theorem 4.2. There are positive numbers $\delta$ and $R_{0}$ such that, for any function $V \in \mathcal{U}_{\delta}$, the family $\left\{\left\|\mathfrak{P}_{t}^{V} \mathbf{1}\right\|_{R}^{-1} \mathfrak{P}_{t}^{V} \psi\right.$, $t \geq 1\}$ is uniformly equicontinuous on $X_{R}$ for any $\psi \in \mathcal{U}$ and $R \geq R_{0}$.

Proof. To prove this result, we develop the arguments of the proof of Theorem 6.2 in [10]. For any $\delta>0, V \in \mathcal{U}_{\delta}$, and $\psi \in \mathcal{U}$, we have

$$
\mathfrak{P}_{t}^{V} \psi(\mathfrak{u})=\mathbb{E}_{\mathfrak{u}}\left\{\left(\Xi_{V} \psi\right)\left(\mathfrak{u}_{t}, t\right)\right\},
$$

where

$$
\left(\Xi_{V} \psi\right)\left(\mathfrak{u}_{t}, t\right):=\exp \left(\int_{0}^{t} V\left(\mathfrak{u}_{\tau}\right) \mathrm{d} \tau\right) \psi\left(\mathfrak{u}_{t}\right)
$$

We prove the uniform equicontinuity of the family $\left\{g_{t}, t \geq 1\right\}$ on $X_{R}$, where

$$
g_{t}(\mathfrak{u})=\left\|\mathfrak{P}_{t}^{V} \mathbf{1}\right\|_{R}^{-1} \mathfrak{P}_{t}^{V} \psi(\mathfrak{u}) .
$$

Without loss of generality, we can assume that $0 \leq \psi \leq 1$ and $\inf _{H} V=0$, so that $\operatorname{Osc}_{H}(V)=\|V\|_{\infty}$. We can assume also that the integer $N$ entering representation (1.10) is the same for $\psi$ and $V$ and it is denoted by $N_{0}$. 
Step 1: Stratification. Let us take any $N \geq N_{0}$ and $\mathfrak{z}, \mathfrak{z}^{\prime} \in X_{R}$ such that $d:=\left|\mathfrak{z}-\mathfrak{z}^{\prime}\right|_{\mathcal{H}} \leq 1$, and denote by $(\Omega, \mathcal{F}, \mathbb{P})$ the probability space constructed in the previous subsection. Let us consider a coupled trajectory $\left(\mathfrak{u}_{t}, \mathfrak{u}_{t}^{\prime}\right):=\left(\tilde{\mathfrak{u}}_{t}, \tilde{\mathfrak{u}}_{t}^{\prime}\right)$ at level $N$ issued from $\left(\mathfrak{z}, \mathfrak{z}^{\prime}\right)$ and the associated process $\mathfrak{v}_{t}:=\tilde{\mathfrak{v}}_{t}$. For any integers $r \geq 0$ and $\rho \geq 1$, we $\operatorname{set}^{7}$

$$
\begin{aligned}
\bar{G}_{r}=\bigcap_{j=0}^{r} G_{j}, \quad G_{j}=\left\{\mathfrak{v}_{t}=\mathfrak{u}_{t}^{\prime}, \forall t \in(j, j+1]\right\}, \quad F_{r, 0}=\varnothing, \\
F_{r, \rho}=\left\{\sup _{\tau \in[0, r]}\left(\int_{0}^{\tau}\left(\left\|\nabla u_{\tau}\right\|^{2}+\left\|\nabla u_{\tau}^{\prime}\right\|^{2}\right) \mathrm{d} \tau-L \tau\right) \leq|\mathcal{E}(\mathfrak{z})|+\left|\mathcal{E}\left(\mathfrak{z}^{\prime}\right)\right|+\rho ;\right. \\
\left.\left|\mathcal{E}\left(\mathfrak{u}_{r}\right)\right|+\left|\mathcal{E}\left(\mathfrak{u}_{r}^{\prime}\right)\right| \leq \rho\right\},
\end{aligned}
$$

where $L$ is the constant in (4.15). We also define the pairwise disjoint events

$$
A_{0}=G_{0}^{c}, \quad A_{r, \rho}=\left(\bar{G}_{r-1} \cap G_{r}^{c} \cap F_{r, \rho}\right) \backslash F_{r, \rho-1}, \quad r \geq 1, \rho \geq 1, \quad \tilde{A}=\bar{G}_{+\infty} .
$$

Then, for any $t \geq 1$, we have

$$
\begin{aligned}
\mathfrak{P}_{t}^{V} \psi(\mathfrak{z})-\mathfrak{P}_{t}^{V} \psi\left(\mathfrak{z}^{\prime}\right)= & \mathbb{E}\left\{\mathbb{I}_{A_{0}}\left[\left(\Xi_{V} \psi\right)\left(\mathfrak{u}_{t}, t\right)-\left(\Xi_{V} \psi\right)\left(\mathfrak{u}_{t}^{\prime}, t\right)\right]\right\} \\
& +\sum_{r, \rho=1}^{\infty} \mathbb{E}\left\{\mathbb{I}_{A_{r, \rho}}\left[\left(\Xi_{V} \psi\right)\left(\mathfrak{u}_{t}, t\right)-\left(\Xi_{V} \psi\right)\left(\mathfrak{u}_{t}^{\prime}, t\right)\right]\right\} \\
& +\mathbb{E}\left\{\mathbb{I}_{\tilde{A}}\left[\left(\Xi_{V} \psi\right)\left(\mathfrak{u}_{t}, t\right)-\left(\Xi_{V} \psi\right)\left(\mathfrak{u}_{t}^{\prime}, t\right)\right]\right\} \\
= & I_{0}^{t}\left(\mathfrak{z}, \mathfrak{z}^{\prime}\right)+\sum_{r, \rho=1}^{\infty} I_{r, \rho}^{t}\left(\mathfrak{z}, \mathfrak{z}^{\prime}\right)+\tilde{I}^{t}\left(\mathfrak{z}, \mathfrak{z}^{\prime}\right),
\end{aligned}
$$

where

$$
\begin{aligned}
& I_{0}^{t}\left(\mathfrak{z}, \mathfrak{z}^{\prime}\right):=\mathbb{E}\left\{\mathbb{I}_{A_{0}}\left[\left(\Xi_{V} \psi\right)\left(\mathfrak{u}_{t}, t\right)-\left(\Xi_{V} \psi\right)\left(\mathfrak{u}_{t}^{\prime}, t\right)\right]\right\}, \\
& I_{r, \rho}^{t}\left(\mathfrak{z}, \mathfrak{z}^{\prime}\right):=\mathbb{E}\left\{\mathbb{I}_{A_{r, \rho}}\left[\left(\Xi_{V} \psi\right)\left(\mathfrak{u}_{t}, t\right)-\left(\Xi_{V} \psi\right)\left(\mathfrak{u}_{t}^{\prime}, t\right)\right]\right\}, \\
& \tilde{I}^{t}\left(\mathfrak{z}, \mathfrak{z}^{\prime}\right):=\mathbb{E}\left\{\mathbb{I}_{\tilde{A}}\left[\left(\Xi_{V} \psi\right)\left(\mathfrak{u}_{t}, t\right)-\left(\Xi_{V} \psi\right)\left(\mathfrak{u}_{t}^{\prime}, t\right)\right]\right\} .
\end{aligned}
$$

To prove the uniform equicontinuity of $\left\{g_{t}, t \geq 1\right\}$, we first estimate these three quantities.

Step 2: Estimates for $I_{0}^{t}$ and $I_{r, \rho}^{t}$. Let $\delta_{1}>0$ and $R_{0} \geq 1$ be the numbers in Proposition 3.1. Then, if $\operatorname{Osc}(V)<\delta_{1}$ and $R \geq R_{0}$, we have the following estimates

$$
\begin{aligned}
& \left|I_{0}^{t}\left(\mathfrak{z}, \mathfrak{z}^{\prime}\right)\right| \leq C_{1}(R, V)\left\|\mathfrak{P}_{t}^{V} \mathbf{1}\right\|_{R} \mathbb{P}\left\{A_{0}\right\}^{1 / 2}, \\
& \left|I_{r, \rho}^{t}\left(\mathfrak{z}, \mathfrak{z}^{\prime}\right)\right| \leq C_{2}(R, V) e^{r\|V\|_{\infty}}\left\|\mathfrak{P}_{t}^{V} \mathbf{1}\right\|_{R} \mathbb{P}\left\{A_{r, \rho}\right\}^{1 / 2}
\end{aligned}
$$

for any integers $r, \rho \geq 1$. Let us prove (4.7), the other estimate is similar. First assume that $r \leq t$. Using the inequalities $0 \leq \psi \leq 1$, the positivity of $\Xi_{V} \psi$, and the Markov property, we derive

$$
\begin{aligned}
I_{r, \rho}^{t}\left(\mathfrak{z}, \mathfrak{z}^{\prime}\right) & \leq \mathbb{E}\left\{I_{A_{r, \rho}}\left(\Xi_{V} \psi\right)\left(\mathfrak{u}_{t}, t\right)\right\} \leq \mathbb{E}\left\{I_{A_{r, \rho}}\left(\Xi_{V} \mathbf{1}\right)\left(\mathfrak{u}_{t}, t\right)\right\} \\
& =\mathbb{E}\left\{I_{A_{r, \rho}} \mathbb{E}\left[\left(\boldsymbol{\Xi}_{V} \mathbf{1}\right)\left(\mathfrak{u}_{t}, t\right) \mid \mathcal{F}_{r}\right]\right\} \leq e^{r\|V\|_{\infty}} \mathbb{E}\left\{I_{A_{r, \rho}}\left(\mathfrak{P}_{t-r}^{V} \mathbf{1}\right)\left(\mathfrak{u}_{r}\right)\right\},
\end{aligned}
$$

${ }^{7}$ The event $\bar{G}_{r}$ is well defined also for $r=+\infty$. 
where $\left\{\mathcal{F}_{t}\right\}$ stands for the filtration generated by $\left(\mathfrak{u}_{t}, \mathfrak{u}_{t}^{\prime}\right)$. Then from (3.1) it follows that

$$
\mathfrak{P}_{t-r}^{V} \mathbf{1}(\mathfrak{z}) \leq M\left\|\mathfrak{P}_{t-r}^{V} \mathbf{1}\right\|_{R_{0}} \mathfrak{w}(\mathfrak{z}),
$$

so we have

$$
\begin{aligned}
I_{r, \rho}^{t}\left(\mathfrak{z}, \mathfrak{z}^{\prime}\right) & \leq C_{3} e^{r\|V\|_{\infty}}\left\|\mathfrak{P}_{t-r}^{V} \mathbf{1}\right\|_{R_{0}} \mathbb{E}\left\{I_{A_{r, \rho}} \mathfrak{w}\left(\mathfrak{u}_{r}\right)\right\} \\
& \leq C_{3} e^{r\|V\|_{\infty}}\left\|\mathfrak{P}_{t-r}^{V} \mathbf{1}\right\|_{R_{0}}\left\{\mathbb{P}\left(A_{r, \rho}\right) \mathbb{E} \mathfrak{w}^{2}\left(\mathfrak{u}_{r}\right)\right\}^{1 / 2} .
\end{aligned}
$$

Using this, (5.24), and the symmetry, we obtain (4.7). If $r>t$, then

$$
I_{r, \rho}^{t}\left(\mathfrak{z}, \mathfrak{z}^{\prime}\right) \leq e^{r\|V\|_{\infty}} \mathbb{P}\left\{A_{r, \rho}\right\} \leq e^{r\|V\|_{\infty}}\left\|\mathfrak{P}_{t}^{V} \mathbf{1}\right\|_{R} \mathbb{P}\left\{A_{r, \rho}\right\}^{1 / 2},
$$

which implies (4.7) by symmetry.

Step 3: Estimate for $\tilde{I}^{t}$. Let us show that, for any $N \geq N_{0}$, we have

$$
\left|\tilde{I}_{\rho}^{t}\left(\mathfrak{z}, \mathfrak{z}^{\prime}\right)\right| \leq C_{4}(\psi, V)\left\|\mathfrak{P}_{t}^{V} \mathbf{1}\right\|_{R} d^{q} .
$$

Indeed, we write

$$
\begin{aligned}
\tilde{I}^{t}\left(\mathfrak{z}, \mathfrak{z}^{\prime}\right)= & \mathbb{E}\left\{\mathbb{I}_{\tilde{A}}\left(\Xi_{V} \mathbf{1}\right)\left(\mathfrak{u}_{t}, t\right)\left[\psi\left(\mathfrak{u}_{t}\right)-\psi\left(\mathfrak{u}_{t}^{\prime}\right)\right]\right\} \\
& +\mathbb{E}\left\{\mathbb{I}_{\tilde{A}}\left[\left(\boldsymbol{\Xi}_{V} \mathbf{1}\right)\left(\mathfrak{u}_{t}, t\right)-\left(\Xi_{V} \mathbf{1}\right)\left(\mathfrak{u}_{t}^{\prime}, t\right)\right] \psi\left(\mathfrak{u}_{t}^{\prime}\right)\right\} .
\end{aligned}
$$

Let us denote by $J_{1, \rho}^{t}$ and $J_{2, \rho}^{t}$ the expectations in the right-hand side of this equality. Then by estimate (A.27), on the event $\tilde{A}$ we have

$$
\left|P_{N}\left(\mathfrak{u}_{\tau}-\mathfrak{u}_{\tau}^{\prime}\right)\right|_{\mathcal{H}}^{2} \leq e^{-\alpha \tau} d^{2}, \quad \tau \in[0, t] .
$$

Since $\psi \in C_{b}^{q}(\mathcal{H})$, we derive from (4.10)

$$
\begin{aligned}
\left|J_{1, \rho}^{t}\right| & \leq \mathbb{E}\left\{\mathbb{I}_{\tilde{A}}\left(\Xi_{V} \mathbf{1}\right)\left(\mathfrak{u}_{t}, t\right)\left|\psi\left(\mathfrak{u}_{t}\right)-\psi\left(\mathfrak{u}_{t}^{\prime}\right)\right|\right\} \leq\|\psi\|_{C_{b}^{q}} e^{-\alpha t / 2} d^{q}\left\|\mathfrak{P}_{t}^{V} \mathbf{1}\right\|_{R} \\
& \leq\|\psi\|_{C_{b}^{q}}\left\|\mathfrak{P}_{t}^{V} \mathbf{1}\right\|_{R} d^{q} .
\end{aligned}
$$

Similarly, as $V \in C_{b}^{q}(\mathcal{H})$,

$$
\begin{aligned}
& \left|J_{2, \rho}^{t}\right| \leq \mathbb{E}\left\{\mathbb{I}_{\tilde{A}}\left|\left(\Xi_{V} \mathbf{1}\right)\left(\mathfrak{u}_{t}, t\right)-\left(\Xi_{V} \mathbf{1}\right)\left(\mathfrak{u}_{t}^{\prime}, t\right)\right|\right\} \\
& \leq \mathbb{E}\left\{\mathbb{I}_{\tilde{A}}\left(\boldsymbol{\Xi}_{V} \mathbf{1}\right)\left(\mathfrak{u}_{t}, t\right)\left[\exp \left(\int_{0}^{t}\left|V\left(\mathfrak{u}_{\tau}\right)-V\left(\mathfrak{u}_{\tau}^{\prime}\right)\right| \mathrm{d} \tau\right)-1\right]\right\} \\
& \leq\left[\exp \left(\|V\|_{C_{b}^{q}} d^{q}\left(1-e^{-\alpha q t / 2}\right)\right)-1\right]\left\|\mathfrak{P}_{t}^{V} \mathbf{1}\right\|_{R} \\
& \leq\left[\exp \left(\|V\|_{C_{b}^{q}} d^{q}\right)-1\right]\left\|\mathfrak{P}_{t}^{V} \mathbf{1}\right\|_{R} .
\end{aligned}
$$

Combining these estimates for $J_{1, \rho}^{t}$ and $J_{2, \rho}^{t}$ with (4.9), we get (4.8).

Step 4: Proof of uniform equicontinuity. We use the following lemma which is proved at the end of this subsection.

Lemma 4.3. For sufficiently large $N \geq 1$, we have

$$
\begin{aligned}
& \mathbb{P}\left\{A_{0}\right\} \leq C_{5}(R, N) d^{a / 2}, \\
& \mathbb{P}\left\{A_{r, \rho}\right\} \leq C_{6}(R)\left\{\left(d^{a} e^{-a \alpha r / 2}+\left[\exp \left(C_{7}(R, N) d^{a} e^{2 \rho-a \alpha r / 2}\right)-1\right]^{1 / 2}\right) \wedge e^{-\beta \rho}\right\},
\end{aligned}
$$

where $a$ and $\beta$ are the constants in (4.2) and (4.15). 
From (4.5)-(4.8), (4.11), and (4.12) it follows that, for any $\mathfrak{z}, \mathfrak{z}^{\prime} \in X_{R}, t \geq 1$, and $R \geq R_{0}$, we have

$$
\begin{aligned}
\left|g_{t}(\mathfrak{z})-g_{t}\left(\mathfrak{z}^{\prime}\right)\right| \leq & C_{8}(R, V, N, \psi)\left(d^{a / 4}+d^{q}\right. \\
& \left.+\sum_{r, \rho=1}^{\infty} e^{r\|V\|_{\infty}}\left\{\left(d^{a / 2} e^{-a \alpha r / 4}+\left[\exp \left(C_{7} d^{a} e^{2 \rho-a \alpha r / 2}\right)-1\right]^{1 / 4}\right) \wedge e^{-\beta \rho / 2}\right\}\right),
\end{aligned}
$$

provided that $N \geq N_{0} \vee N_{1} \vee N_{2}$. When $d=0$, the series in the right-hand side vanishes. So to prove the uniform equicontinuity of $\left\{g_{t}\right\}$, it suffices to show that the series converges uniformly in $d \in[0,1]$. Since its terms are positive and monotone, it suffices to show the converge for $d=1$ :

$$
\sum_{r, \rho=1}^{\infty} e^{r\|V\|_{\infty}}\left\{\left(e^{-a \alpha r / 4}+\left[\exp \left(C_{7} e^{2 \rho-a \alpha r / 2}\right)-1\right]^{1 / 4}\right) \wedge e^{-\beta \rho / 2}\right\}<\infty .
$$

To prove this, we will assume that $\operatorname{Osc}(V)$ is sufficiently small. Let us consider the sets

$$
S_{1}=\left\{(r, \rho) \in \mathbb{N}^{2}: \rho \leq a \alpha r / 8\right\}, \quad S_{2}=\mathbb{N}^{2} \backslash S_{1} .
$$

Then taking $\delta<\delta_{1} \vee(a \alpha / 32)$ and $\operatorname{Osc}(V)<\delta$, we see that

$$
\begin{aligned}
& \sum_{(r, \rho) \in S_{1}} e^{r\|V\|_{\infty}}\left(e^{-a \alpha r / 4}+\left[\exp \left(C_{7} e^{2 \rho-a \alpha r / 2}\right)-1\right]^{1 / 4}\right) \\
& \leq C_{9}(R, N) \sum_{(r, \rho) \in S_{1}} e^{r\|V\|_{\infty}} e^{-a \alpha r / 16} \leq C_{10}(R, N) \sum_{r=1}^{\infty} e^{-a \alpha r / 32}<\infty .
\end{aligned}
$$

Choosing $\delta<a \alpha \beta / 32$, we get

$$
\sum_{(r, \rho) \in S_{2}} e^{r\|V\|_{\infty}} e^{-\beta \rho / 2} \leq C_{11} \sum_{\rho=1}^{\infty} e^{-\beta \rho / 4}<\infty .
$$

These two inequalities show that (4.13) holds.

Proof of Lemma 4.3. Taking $\varepsilon=d$ in (4.3), using (3.8), and recalling that $d \leq 1$, we get

$$
\mathbb{P}\left\{A_{0}\right\} \leq C_{*} d^{a}+C_{*}\left[\exp \left(C_{N} d^{a} e^{C_{7} R^{4}}\right)-1\right]^{1 / 2} \leq C_{5}(R, N) d^{a / 2},
$$

provided that $N$ is larger that the number $N_{1}$ in Proposition 4.1. This gives (4.11). To show (4.12), we use the estimates

$$
\begin{aligned}
& \mathbb{E}_{\mathfrak{u}} \exp \left(\beta\left|\mathcal{E}\left(\mathfrak{u}_{t}\right)\right|\right) \leq C \exp (\beta|\mathcal{E}(\mathfrak{u})|), \quad \mathfrak{u} \in \mathcal{H}, \\
& \mathbb{P}_{\mathfrak{u}}\left\{\sup _{t \geq 0}\left(\int_{0}^{t}\left\|\nabla u_{\tau}\right\|^{2} \mathrm{~d} \tau-L t\right) \geq|\mathcal{E}(\mathfrak{u})|+\rho\right\} \leq C e^{-\beta \rho}, \quad \rho>0,
\end{aligned}
$$

where $L, \beta$, and $C$ are some positive constants depending on $\gamma,\|h\|$, and $B_{0}$; they follow immediately from Propositions 3.1 and 3.2 in [21]. From the inclusion $A_{r, \rho} \subset F_{r, \rho-1}^{c}$ and inequalities (4.14), (4.15), and (3.8) it follows that

$$
\mathbb{P}\left\{A_{r, \rho}\right\} \leq C_{12}(R) e^{-\beta \rho} .
$$

By the Foiaş-Prodi type estimate (see (A.29) in Proposition A.5), there is $N_{2} \geq 1$ such that for any $N \geq N_{2}$ on the event $\bar{G}_{r-1} \cap F_{r, \rho}$ we have

$$
\left|\mathfrak{u}_{r}-\mathfrak{u}_{r}^{\prime}\right|_{\mathcal{H}}^{2} \leq \exp \left(-\alpha r+\rho+|\mathcal{E}(\mathfrak{z})|+\left|\mathcal{E}\left(\mathfrak{z}^{\prime}\right)\right|\right) d^{2} \leq C_{13}(R) e^{-\alpha r+\rho} d^{2}
$$


where we used (3.8). Recall that on the same event we have also

$$
\left|\mathcal{E}\left(\mathfrak{u}_{r}\right)\right|+\left|\mathcal{E}\left(\mathfrak{u}_{r}^{\prime}\right)\right| \leq \rho .
$$

So using the Markov property, (4.3) with $\varepsilon=d e^{-\alpha r / 2},(4.18)$ and (4.17), we obtain

$$
\begin{aligned}
\mathbb{P}\left\{A_{r, \rho}\right\} \leq & \left.\mathbb{P}\left\{\bar{G}_{r-1}\right) \cap G_{r}^{c} \cap F_{r, \rho}\right\}=\mathbb{E}\left\{\mathbb{I}_{\bar{G}_{r-1} \cap F_{r, \rho}} \mathbb{E}\left(I_{G_{r}^{c}} \mid \mathcal{F}_{r}\right)\right\} \\
\leq & C_{*} d^{a} e^{-a \alpha r / 2}+C_{*} \mathbb{E}\left\{\mathbb{I}_{\bar{G}_{r-1} \cap F_{r, \rho}}\right. \\
& \left.\times\left[\exp \left(C_{N} d^{a-2} e^{-(a-2) \alpha r / 2}\left|\mathfrak{u}_{r}-\mathfrak{u}_{r}^{\prime}\right|_{\mathcal{H}^{2}}^{2} e^{\left(\left|\mathcal{E}\left(\mathfrak{u}_{r}\right)\right|+\left|\mathcal{E}\left(\mathfrak{u}_{r}^{\prime}\right)\right|\right)}\right)-1\right]^{1 / 2}\right\} \\
\leq & C_{*} d^{a} e^{-a \alpha r / 2}+C_{*}\left[\exp \left(C_{6}(R, N) d^{a} e^{2 \rho-a \alpha r / 2}\right)-1\right]^{1 / 2} .
\end{aligned}
$$

Combining this with (4.16) and choosing $N \geq N_{1} \vee N_{2}$, we get the required inequality (4.12).

\section{Estimates for regular solutions}

In this section, we establish the exponential tightness property and obtain some higher order moment estimates for solutions in $\mathcal{H}^{r}$.

\subsection{Exponential tightness}

Here we show that the exponential tightness property (1.19) is verified for the function $\Phi(\mathfrak{u})=|\mathfrak{u}|_{\mathcal{H}^{r}}^{\varkappa}$, if we choose $\varkappa>0$ sufficiently small. Clearly, the level sets of $\Phi$ are compact in $\mathcal{H}$.

Theorem 5.1. For any $r<1 / 2$, there is $\varkappa \in(0,1)$ such that, for any $R \geq 1$, we have

$$
\mathbb{E}_{\mathfrak{v}} \exp \left(\int_{0}^{t}\left|\mathfrak{u}_{\tau}\right|_{\mathcal{H}^{r}}^{\mathcal{H}} \mathrm{d} \tau\right) \leq c e^{c t} \quad \text { for any } \mathfrak{v} \in X_{R}, t \geq 0
$$

where $c$ is a positive constant depending on $R$.

Proof. It is sufficient to prove that there is $\varkappa \in(0,1)$ such that, for any $R \geq 1$, we have

$$
\mathbb{E}_{\mathfrak{v}} \exp \left(\delta \int_{0}^{t}\left|\mathfrak{u}_{\tau}\right|_{\mathcal{H}^{r}}^{\varkappa} \mathrm{d} \tau\right) \leq \tilde{c} e^{\tilde{c} t} \quad \text { for any } \mathfrak{v} \in X_{R}, t \geq 0
$$

where $\delta$ and $\tilde{c}$ are positive constants depending on $R$. Indeed, once this is proved, we can use the inequality

$$
|\mathfrak{u}|_{\mathcal{H}^{r}}^{\frac{\varkappa}{2}} \leq \delta|\mathfrak{u}|_{\mathcal{H}^{r}}^{\varkappa}+\delta^{-1}
$$

to derive (5.1), where $\varkappa$ should be replaced by $\varkappa / 2$. We divide the proof of (5.2) into several steps.

Step 1: Reduction. Let us split the flow $\mathfrak{u}(t)$ to the sum $\mathfrak{u}=\mathfrak{v}_{1}+\mathfrak{v}_{2}+\mathfrak{z}$, where $\mathfrak{v}_{1}(t)=\left[v_{1}(t), \dot{v}_{1}(t)\right]$ corresponds to the flow of (0.1) with $f=h=\vartheta=0$ issued from $\mathfrak{v}$ and $\mathfrak{v}_{2}(t)=\left[v_{2}(t), \dot{v}_{2}(t)\right]$ is the flow of $(0.1)$ with $f=0$ issued from the origin. Some standard arguments show that the following a priori estimates hold:

$$
\begin{aligned}
& \left|\mathfrak{v}_{1}(t)\right|_{\mathcal{H}^{r}}^{2} \leq|\mathfrak{v}|_{\mathcal{H}^{r}}^{2} e^{-\alpha t}, \\
& \mathbb{E} \exp \left(\delta_{1} \int_{0}^{t}\left|\mathfrak{v}_{2}(\tau)\right|_{\mathcal{H}^{r}}^{2} \mathrm{~d} \tau\right) \leq c_{1} e^{c_{1} t} \quad \text { for any } t \geq 0,
\end{aligned}
$$


where $\delta_{1}$ and $c_{1}$ are positive constants depending only on $\alpha, B_{1}$, and $\|h\|_{1}$. Now using the Cauchy-Schwarz inequality and (5.3), we get, for any $\delta<\delta_{1} / 2$,

$$
\begin{aligned}
\mathbb{E}_{\mathfrak{v}} \exp \left(\delta \int_{0}^{t}|\mathfrak{u}(\tau)|_{\mathcal{H}^{r}}^{\varkappa} \mathrm{d} \tau\right) \leq & \exp \left(\delta \int_{0}^{t}\left|\mathfrak{v}_{1}(\tau)\right|_{\mathcal{H}^{r}}^{\varkappa} \mathrm{d} \tau\right) \mathbb{E} \exp \left(2 \delta \int_{0}^{t}\left|\mathfrak{v}_{2}(\tau)\right|_{\mathcal{H}^{r}}^{\varkappa} \mathrm{d} \tau\right) \\
& \times \mathbb{E} \exp \left(2 \delta \int_{0}^{t}|\mathfrak{z}(\tau)|_{\mathcal{H}^{r}}^{\varkappa} \mathrm{d} \tau\right) \\
\leq & \exp \left(2 \delta R^{\varkappa}(\alpha \varkappa)^{-1}\right) \mathbb{E} \exp \left(2 \delta \int_{0}^{t}\left(\left|\mathfrak{v}_{2}(\tau)\right|_{\mathcal{H}^{r}}^{2}+1\right) \mathrm{d} \tau\right) \\
& \times \mathbb{E} \exp \left(2 \delta \int_{0}^{t}|\mathfrak{z}(\tau)|_{\mathcal{H}^{r}}^{\varkappa} \mathrm{d} \tau\right) .
\end{aligned}
$$

Combining this with (5.4), we see that inequality (5.2) will be established if we prove that

$$
\mathbb{E} \exp \left(\delta \int_{0}^{t}|\mathfrak{z}(\tau)|_{\mathcal{H}^{r}}^{\varkappa} \mathrm{d} \tau\right) \leq c e^{c t} \quad \text { for all } t \geq 0
$$

for some $\delta>0$ and $c>0$. The rest of the proof is devoted to the derivation of this inequality.

Step 2: Pointwise estimates. Let us note that, by construction, $\mathfrak{z}$ is the flow of equation

$$
\partial_{t}^{2} z+\gamma \partial_{t} z-\Delta z+f(u)=0,\left.\quad z\right|_{\partial D}=0,[z(0), \dot{z}(0)]=0 .
$$

Let us differentiate this equation in time, and set $a=\dot{z}(t)$. Then $a$ solves

$$
\partial_{t}^{2} a+\gamma \partial_{t} a-\Delta a+f^{\prime}(u) \partial_{t} u=0,\left.\quad a\right|_{\partial D}=0,[a(0), \dot{a}(0)]=[0,-f(u(0))] .
$$

We write $\mathfrak{a}(t)=[a(t), \dot{a}(t)]$. Multiplying equation (5.7) by $2(-\Delta)^{r-1}(\dot{a}+\alpha a)$ and integrating over $D$, we obtain

$$
\frac{\mathrm{d}}{\mathrm{d} t}|\mathfrak{a}|_{\mathcal{H}^{r-1}}^{2}+\frac{3 \alpha}{2}|\mathfrak{a}|_{\mathcal{H}^{r-1}}^{2} \leq 2 \int_{D}\left|f^{\prime}(u)\right||\dot{u}|\left|(-\Delta)^{s-1}(\dot{a}+\alpha a)\right| \mathrm{d} x=\mathcal{L} .
$$

Let $\varkappa<1$ be a positive constant that will be fixed later. Then, by the triangle inequality, we have

$$
\begin{aligned}
\frac{\mathcal{L}}{2} \leq & \int_{D}\left|f^{\prime}(u)\right|\left|\dot{v}_{1}\right|^{1-\varkappa}|\dot{u}|^{\varkappa}\left|(-\Delta)^{r-1}(\dot{a}+\alpha a)\right| \mathrm{d} x \\
& +\int_{D}\left|f^{\prime}(u)\right|\left|\dot{v}_{2}\right|^{1-\varkappa}|\dot{u}|^{\varkappa}\left|(-\Delta)^{r-1}(\dot{a}+\alpha a)\right| \mathrm{d} x \\
& +\int_{D}\left|f^{\prime}(u)\right||a|^{1-\varkappa}|\dot{u}|^{\varkappa}\left|(-\Delta)^{r-1}(\dot{a}+\alpha a)\right| \mathrm{d} x=\mathcal{L}_{1}+\mathcal{L}_{2}+\mathcal{L}_{3} .
\end{aligned}
$$

Using the Hölder inequality, we derive

$$
\begin{aligned}
& \mathcal{L}_{1} \leq\left|f^{\prime}(u)\right|_{L^{p_{1}}}\left|\dot{v}_{1}\right|_{L^{(1-\varkappa) p_{2}}}^{1-\varkappa}|\dot{u}|_{L^{\varkappa p_{3}}}^{\varkappa}\left|(-\Delta)^{r-1}(\dot{a}+\alpha a)\right|_{L^{p_{4}}}, \\
& \mathcal{L}_{2} \leq\left|f^{\prime}(u)\right|_{L^{q_{1}}}\left|\dot{v}_{2}\right|_{L^{(1-\varkappa) q_{2}}}^{1-\varkappa}|\dot{u}|_{L^{\varkappa q_{3}}}^{\varkappa}\left|(-\Delta)^{r-1}(\dot{a}+\alpha a)\right|_{L^{q_{4}}}, \\
& \mathcal{L}_{3} \leq\left|f^{\prime}(u)\right|_{L^{p_{1}}}|a|_{L^{(1-\varkappa) p_{2}}}^{1-\varkappa}|\dot{u}|_{L^{\varkappa p_{3}}}^{\varkappa}\left|(-\Delta)^{r-1}(\dot{a}+\alpha a)\right|_{L^{p_{4}}},
\end{aligned}
$$

where the exponents $p_{i}, q_{i}$ are Hölder admissible. We now need the following lemma, which is established in the Appendix. 
Lemma 5.2. Let us take $p_{1}=6 / \rho, p_{3}=2 / \varkappa, q_{1}=(\rho+2) / \rho$ and $q_{3}=2 / \varkappa$. Then, for $\varkappa>0$ sufficiently small, the exponents $p_{2}, p_{4}, q_{2}$ and $q_{4}$ can be chosen in such a way that we have the following embeddings:

$$
\begin{aligned}
& 2 H^{r} \hookrightarrow L^{(1-\varkappa) p_{2}}, \quad H^{1-r} \hookrightarrow L^{p_{4}}, \\
& H^{1} \hookrightarrow L^{(1-\varkappa) q_{2}}, \quad H^{1-r} \hookrightarrow L^{q_{4}} .
\end{aligned}
$$

Step 3: Estimation of $\mathcal{L}_{1}$ and $\mathcal{L}_{3}$. In view of Lemma 5.2 and inequalities (1.1) and (5.10), we have

$$
\begin{aligned}
\mathcal{L}_{1} & \leq C_{0}\left|f^{\prime}(u)\right|_{L^{6 / \rho}}\left\|\dot{v}_{1}\right\|_{r}^{1-\varkappa}\|\dot{u}\|^{\varkappa}\left\|(-\Delta)^{r-1}(\dot{a}+\alpha a)\right\|_{1-r} \\
& \leq C_{1}\left\|\dot{v}_{1}\right\|_{r}^{1-\varkappa}\left(\|u\|_{1}^{\rho}+1\right)\|\dot{u}\|^{\varkappa}\|\dot{a}+\alpha a\|_{r-1} .
\end{aligned}
$$

Now let us suppose that $\varkappa<2-\rho$. Then using (5.3) together with the Young inequality, we derive

$$
\mathcal{L}_{1} \leq C_{2}|\mathfrak{v}|_{\mathcal{H}^{r}}^{1-\varkappa}\left(\|u\|_{1}^{2}+\|\dot{u}\|^{2}+C_{\varkappa}\right)\|\dot{a}+\alpha a\|_{r-1} \leq C_{3} R\left(\mathcal{E}(\mathfrak{u})+C_{3}\right)|\mathfrak{a}|_{\mathcal{H}^{r-1}}
$$

To estimate $\mathcal{L}_{3}$, we again apply Lemma 5.2 and inequalities (1.1) and (5.12)

$$
\mathcal{L}_{3} \leq C_{4}\left(\|u\|_{1}^{\rho}+1\right)\|a\|_{r}^{1-\varkappa}\|\dot{u}\|^{\varkappa}\|\dot{a}+\alpha a\|_{r-1} \leq C_{4}\left(\|u\|_{1}^{\rho}+1\right)\|\dot{u}\|^{\varkappa}|\mathfrak{a}|_{\mathcal{H}^{r-1}}^{2-\varkappa}
$$

Applying the Young inequality, we get

$$
\mathcal{L}_{3} \leq C_{5}\left(\mathcal{E}(\mathfrak{u})+C_{5}\right)|\mathfrak{a}|_{\mathcal{H}^{r-1}}^{2-\varkappa} .
$$

Step 4: Estimation of $\mathcal{L}_{2}$. It follows from Lemma 5.2 and inequalities (1.2) and (5.11) that

$$
\begin{aligned}
\mathcal{L}_{2} & \leq C_{6}\left|f^{\prime}(u)\right|_{L^{(\rho+2) / \rho}}\left\|\dot{v}_{2}\right\|_{1}^{1-\varkappa}\|\dot{u}\|^{\varkappa}\left\|(-\Delta)^{r-1}(\dot{a}+\alpha a)\right\|_{1-r} \\
& \leq C_{7}\left\|\dot{v}_{2}\right\|_{1}^{1-\varkappa}\left(\int_{D}\left(F(u)+v u^{2}+C\right) \mathrm{d} x\right)^{\rho / \rho+2}\|\dot{u}\|^{\varkappa}\|\dot{a}+\alpha a\|_{r-1} \\
& \leq C_{8}\left\|\dot{v}_{2}\right\|_{1}^{1-\varkappa}\left(\mathcal{E}(\mathfrak{u})+C_{8}\right)^{\rho / \rho+2}\|\dot{u}\|^{\varkappa}|\mathfrak{a}|_{\mathcal{H}^{r-1}} .
\end{aligned}
$$

Finally, applying the Young inequality, we obtain

$$
\mathcal{L}_{2} \leq C_{9}\left(\mathcal{E}(\mathfrak{u})+\left|\mathfrak{v}_{2}\right|_{\mathcal{H}^{s}}^{2}+C_{9}\right)|\mathfrak{a}|_{\mathcal{H}^{r-1}}
$$

Step 5: Estimation of $|\mathfrak{a}|_{\mathcal{H}}^{r-1}$. Combining inequalities (5.8), (5.9) and (5.15)-(5.17), we see that

$$
\frac{\mathrm{d}}{\mathrm{d} t}|\mathfrak{a}(t)|_{\mathcal{H}^{r-1}}^{2}+\alpha|\mathfrak{a}(t)|_{\mathcal{H}^{r-1}}^{2} \leq C_{10} R\left(\mathcal{E}(\mathfrak{u}(t))+\left|\mathfrak{v}_{2}(t)\right|_{\mathcal{H}^{s}}^{2}+C_{10}\right)\left(|\mathfrak{a}(t)|_{\mathcal{H}^{r-1}}^{2-\varkappa}+1\right) .
$$

We now need an auxiliary result, whose proof is presented in the Appendix.

Lemma 5.3. Let $x(t)$ be an absolutely continuous nonnegative function satisfying the differential inequality

$$
\dot{x}(t)+\alpha x(t) \leq g(t) x^{1-\beta}(t)+b(t) \quad \text { for all } t \in[0, T],
$$

where $\alpha, T$, and $\beta<1$ are positive constants and $g(t)$ and $b(t)$ are nonnegative functions integrable on $[0, T]$. Then we have

$$
\frac{\alpha}{2} \int_{0}^{t} x^{\beta}(\tau) \mathrm{d} \tau \leq \beta^{-1}(1+x(0))^{\beta}+\int_{0}^{t}(\alpha+g(\tau)+b(\tau)) \mathrm{d} \tau \quad \text { for } t \in[0, T] .
$$


Applying this lemma to inequality (5.18), we obtain

$$
\begin{aligned}
\frac{\alpha}{2} \int_{0}^{t}|\mathfrak{a}(\tau)|_{\mathcal{H}^{r-1}}^{\varkappa} \mathrm{d} \tau \leq & 2 \varkappa^{-1}\left(1+|\mathfrak{a}(0)|_{\mathcal{H}^{r-1}}^{2}\right)^{\varkappa / 2}+\alpha t \\
& +2 C_{10} R \int_{0}^{t}\left(\mathcal{E}(\mathfrak{u}(\tau))+\left|\mathfrak{v}_{2}(\tau)\right|_{\mathcal{H}^{s}}^{2}+C_{10}\right) \mathrm{d} \tau .
\end{aligned}
$$

Step 6: Completion of the proof. Note that

$$
|\mathfrak{z}|_{\mathcal{H}^{r}}^{2}=\|z\|_{r+1}^{2}+\|\dot{z}+\alpha z\|_{r}^{2}=\|\Delta z\|_{r-1}^{2}+\|a+\alpha z\|_{r}^{2} .
$$

On the other, in view of (5.6), we have

$$
\|\Delta z\|_{r-1}^{2}=\|\dot{a}+\gamma a+f(u)\|_{r-1}^{2} \leq C_{11}\left(|\mathfrak{a}|_{\mathcal{H}^{r-1}}^{2}+\|f(u)\|^{2}\right),
$$

whence we get

$$
|\mathfrak{z}|_{\mathcal{H}^{r}}^{2} \leq C_{12}\left(|\mathfrak{a}|_{\mathcal{H}^{r-1}}^{2}+\mathcal{E}^{3}(\mathfrak{u})+C_{12}\right) .
$$

It follows that

$$
|\mathfrak{z}|_{\mathcal{H}^{r}}^{\varkappa} \leq C_{13}\left(|\mathfrak{a}|_{\mathcal{H}^{r-1}}^{\varkappa}+\mathcal{E}(\mathfrak{u})+C_{13}\right),
$$

provided $\varkappa<2 / 3$. Multiplying this inequality by $\alpha / 2$, integrating over $[0, t]$ and using (5.21) together with the fact that

$$
|\mathfrak{a}(0)|_{\mathcal{H}^{r-1}}^{2}=\|f(u(0))\|_{r-1}^{2} \leq\|f(u(0))\|^{2} \leq C_{14}\left(\|\mathfrak{v}\|_{1}^{6}+1\right),
$$

we derive

$$
\frac{\alpha}{2} \int_{0}^{t}|\mathfrak{z}(\tau)|_{\mathcal{H}^{r}}^{\varkappa} \mathrm{d} \tau \leq C_{15}\left(1+\int_{0}^{t}\left[\mathcal{E}(\mathfrak{u}(\tau))+\left|\mathfrak{v}_{2}(\tau)\right|_{\mathcal{H}^{r}}^{2}+C_{15}\right] \mathrm{d} \tau\right)
$$

where $C_{15}$ depends on $R$. Multiplying this inequality by a small constant $\delta(R)>0$, taking the exponent and then the expectation, and using (5.4) together with Proposition 3.2 in [21], we derive (5.5). This completes the proof of (1.19).

\subsection{Higher moments of regular solutions}

For any $m \geq 1$, let $\mathfrak{w}_{m}$ and $\tilde{\mathfrak{w}}_{m}$ be the functions given by (3.19) and (3.20). The following result shows that they are both Lyapunov functions for the trajectories of problem (0.1), (0.3).

Proposition 5.4. For any $\mathfrak{v} \in \mathcal{H}^{r}, m \geq 1$, and $t \geq 0$, we have

$$
\begin{aligned}
& \mathbb{E}_{\mathfrak{v}} \mathfrak{w}_{m}\left(\mathfrak{u}_{t}\right) \leq 2 e^{-\alpha m t} \mathfrak{w}_{m}(\mathfrak{v})+C_{m}, \\
& \mathbb{E}_{\mathfrak{v}} \tilde{\mathfrak{w}}_{m}\left(\mathfrak{u}_{t}\right) \leq 2 e^{-\alpha m t} \tilde{\mathfrak{w}}_{m}(\mathfrak{v})+C_{m} .
\end{aligned}
$$

Proof. Step 1: Proof of (5.24). We split the flow $\mathfrak{u}(t ; \mathfrak{v})$ to the sum $\mathfrak{u}(t ; \mathfrak{v})=\tilde{\mathfrak{u}}(t)+\mathfrak{z}(t)$, where $\tilde{\mathfrak{u}}$ is the flow issued from $\mathfrak{v}$ corresponding to the solution of $(0.1)$ with $f=0$. Let us note that here $\mathfrak{z}=[z, \dot{z}]$ is the same as in Section 5.1. A standard argument shows that

$$
\mathbb{E}|\tilde{\mathfrak{u}}(t)|_{\mathcal{H}^{r}}^{2 m} \leq e^{-\alpha m t}|\mathfrak{v}|_{\mathcal{H}^{r}}^{2 m}+C\left(m,\|h\|_{1}, B_{1}\right)
$$


As in Section 5.1, we set $a=\dot{z}$ and write $\mathfrak{a}=[a, \dot{a}]$. Notice that thanks to the Hölder inequality, the Sobolev embeddings $H^{1} \hookrightarrow L^{6}$ and $H^{1-r} \hookrightarrow L^{6 /(3-\rho)}$ for $r<1-\rho / 2$, and inequality (A.33), we can estimate the right-hand side of inequality (5.8) by

$$
\begin{aligned}
\mathcal{L} & \leq C_{1}\left(|u|_{L^{6}}^{\rho}+1\right)\|\dot{u}\|\left|(-\Delta)^{r-1}(\dot{a}+\alpha a)\right|_{L^{6 /(3-\rho)}} \\
& \leq C_{2}\left(\|u\|_{1}^{2}+1\right)\|\dot{u}\|\left\|(-\Delta)^{r-1}(\dot{a}+\alpha a)\right\|_{1-r} \leq C_{3}\left(|\mathfrak{u}|_{\mathcal{H}}^{3}+1\right)\|\dot{a}+\alpha a\|_{r-1} \\
& \leq \frac{\alpha}{4}|\mathfrak{a}|_{\mathcal{H}^{r-1}}^{2}+C_{4}\left(\mathcal{E}^{3}(\mathfrak{u})+C_{4}\right) .
\end{aligned}
$$

Combining this with (5.8), we infer

$$
\frac{\mathrm{d}}{\mathrm{d} t}|\mathfrak{a}|_{\mathcal{H}^{r-1}}^{2} \leq-\frac{5 \alpha}{4}|\mathfrak{a}|_{\mathcal{H}^{r-1}}^{2}+C_{4}\left(\mathcal{E}^{3}(\mathfrak{u})+C_{4}\right)
$$

It follows that ${ }^{8}$

$$
\frac{\mathrm{d}}{\mathrm{d} t}|\mathfrak{a}|_{\mathcal{H}^{r-1}}^{2 m}=m|\mathfrak{a}|_{\mathcal{H}^{r-1}}^{2 m-2} \frac{\mathrm{d}}{\mathrm{d} t}|\mathfrak{a}|_{\mathcal{H}^{r-1}}^{2} \leq-\alpha m|\mathfrak{a}|_{\mathcal{H}^{r-1}}^{2 m}+C_{5}\left(\mathcal{E}^{3 m}(\mathfrak{u})+C_{5}\right)
$$

where we used the Young inequality. Taking the mean value in this inequality and applying the comparison principle, we derive

$$
\mathbb{E}|\mathfrak{a}(t)|_{\mathcal{H}^{r-1}}^{2 m} \leq e^{-\alpha m t}|\mathfrak{a}(0)|_{\mathcal{H}^{r-1}}^{2 m}+C_{6} \int_{0}^{t} e^{\alpha m(\tau-t)}\left(\mathbb{E} \mathcal{E}^{3 m}(\mathfrak{u}(\tau))+C_{6}\right) \mathrm{d} \tau
$$

Combining this with (5.22) and (5.23), we get

$$
\mathbb{E}|\mathfrak{z}(t)|_{\mathcal{H}^{r}}^{2 m} \leq C_{7}\left(e^{-\alpha m t} \mathcal{E}^{3 m}(\mathfrak{v})+\int_{0}^{t} e^{\alpha m(\tau-t)} \mathbb{E} \mathcal{E}^{3 m}(\mathfrak{u}(\tau)) \mathrm{d} \tau+C_{7}\right)
$$

Using the Itô formula, it is not difficult to show (cf. Proposition 3.1 in [21]) that

$$
\mathbb{E} \mathcal{E}^{k}(\mathfrak{u}(t)) \leq \exp (-\alpha k t) \mathcal{E}^{k}(\mathfrak{v})+C\left(k,\|h\|, B_{0}\right) \text { for any } k \geq 1 .
$$

It follows from the last two inequalities that

$$
\mathbb{E}|\mathfrak{z}(t)|_{\mathcal{H}^{r}}^{2 m} \leq C_{8}\left(e^{-\alpha m t} \mathcal{E}^{3 m}(\mathfrak{v})+C_{8}\right) .
$$

Combining this with the inequality

$$
(x+y)^{2 m} \leq 2 x^{2 m}+C_{9} y^{2 m} \text { for any } x, y \geq 0,
$$

and (5.26), we infer

$$
\begin{aligned}
\mathbb{E}|\mathfrak{u}(t)|_{\mathcal{H}^{r}}^{2 m} & \leq \mathbb{E}\left(|\tilde{\mathfrak{u}}(t)|_{\mathcal{H}^{r}}+|\mathfrak{z}(t)|_{\mathcal{H}^{r}}\right)^{2 m} \leq 2 \mathbb{E}|\tilde{\mathfrak{u}}(t)|_{\mathcal{H}^{r}}^{2 m}+C_{9} \mathbb{E}|\mathfrak{z}(t)|_{\mathcal{H}^{r}}^{2 m} \\
& \leq 2 e^{-\alpha m t}|\mathfrak{v}|_{\mathcal{H}^{r}}^{2 m}+C_{10}\left(e^{-\alpha m t} \mathcal{E}^{3 m}(\mathfrak{v})+C_{10}\right)
\end{aligned}
$$

So that we have

$$
\begin{aligned}
\mathbb{E} \mathfrak{w}_{m}(\mathfrak{u}(t)) & \leq 2 e^{-\alpha m t}|\mathfrak{v}|_{\mathcal{H}^{r}}^{2 m}+C_{10}\left(e^{-\alpha m t} \mathcal{E}^{3 m}(\mathfrak{v})+C_{10}\right)+\mathbb{E} \mathcal{E}^{4 m}(\mathfrak{u}(t)) \\
& \leq 2 e^{-\alpha m t}\left(|\mathfrak{v}|_{\mathcal{H}^{r}}^{2 m}+\mathcal{E}^{4 m}(\mathfrak{v})\right)+C_{11}=2 e^{-\alpha m t} \mathfrak{w}_{m}(\mathfrak{v})+C_{11},
\end{aligned}
$$

where we used the Young inequality together with (5.27).

${ }^{8}$ All the constants $C_{i}, i \geq 5$ depend on $m$. 
Step 2: Proof of (5.25). It was shown in Section 3.2 of [21], that for any $\varkappa \leq(2 \alpha)^{-1} B_{0}$, we have

$$
\begin{aligned}
\mathbb{E}_{\mathfrak{v}} \exp [\varkappa \mathcal{E}(\mathfrak{u}(t))] \leq & \exp (\varkappa \mathcal{E}(\mathfrak{v})) \\
& +\varkappa \int_{0}^{t} \mathbb{E}_{\mathfrak{v}} \exp [\varkappa \mathcal{E}(\mathfrak{u}(\tau))]\left(-\alpha \mathcal{E}(\mathfrak{u}(\tau))+C\left(B_{0},\|h\|\right)\right) \mathrm{d} \tau .
\end{aligned}
$$

Using this with inequality

$$
e^{r}(-\alpha r+C) \leq-\alpha m e^{r}+C_{12} \text { for any } r \geq-C
$$

and applying the Gronwall lemma, we see that

$$
\mathbb{E}_{\mathfrak{v}} \exp [\varkappa \mathcal{E}(\mathfrak{u}(t))] \leq e^{-\alpha m t} \exp (\varkappa \mathcal{E}(\mathfrak{v}))+C_{13} .
$$

Finally, combining this inequality with (5.24), we arrive at (5.25).

\section{Proof of Theorem 1.2}

The results of Sections 3-5 imply that the growth conditions, the uniform irreducibility and uniform Feller properties in Theorem A.4 are satisfied if we take

$$
\begin{aligned}
& X=\mathcal{H}, \quad X_{R}=B_{\mathcal{H}^{r}}(R), \quad P_{t}^{V}(\mathfrak{u}, \Gamma)=\left(\mathfrak{P}_{t}^{V *} \delta_{\mathfrak{u}}\right)(\Gamma), \\
& \mathfrak{w}(\mathfrak{u})=1+|\mathfrak{u}|_{\mathcal{H}^{r}}^{2}+\mathcal{E}^{4}(\mathfrak{u}), \quad \mathcal{C}=\mathcal{U}, \quad V \in \mathcal{U}_{\delta}
\end{aligned}
$$

for sufficiently large integer $R_{0} \geq 1$, small $\delta>0$, and any $r \in(0,1-\rho / 2)$. Let us show that the time-continuity property is also verified.

Step 1: Time-continuity property. We need to show that the function $t \mapsto \mathfrak{P}_{t}^{V} g(\mathfrak{u})$ is continuous from $\mathbb{R}_{+}$to $\mathbb{R}$ for any $g \in C_{\mathfrak{w}}\left(\mathcal{H}^{r}\right)$ and $\mathfrak{u} \in \mathcal{H}^{r}$ (recall that $X_{\infty}=\mathcal{H}^{r}$ ). For any $T, t \geq 0$ and $\mathfrak{u} \in \mathcal{H}^{r}$, we have

$$
\begin{aligned}
\mathfrak{P}_{T}^{V} g(\mathfrak{u})-\mathfrak{P}_{t}^{V} g(\mathfrak{u}) & =\mathbb{E}_{\mathfrak{u}}\left\{\left[\Xi_{V}(T)-\Xi_{V}(t)\right] g\left(\mathfrak{u}_{t}\right)\right\}+\mathbb{E}_{\mathfrak{u}}\left\{\left[g\left(\mathfrak{u}_{T}\right)-g\left(\mathfrak{u}_{t}\right)\right] \Xi_{V}(T)\right\} \\
& =: S_{1}+S_{2},
\end{aligned}
$$

where $\Xi_{V}$ is defined by (3.4). As $V$ is bounded and $g \in C_{\mathfrak{w}}\left(\mathcal{H}^{r}\right)$, we see that

$$
\begin{aligned}
\left|S_{1}\right| & \leq \mathbb{E}_{\mathfrak{u}}\left\{\left|\exp \left(\int_{t}^{T} V\left(\mathfrak{u}_{\tau}\right) \mathrm{d} \tau\right)-1\right| \Xi_{V}(t)\left|g\left(\mathfrak{u}_{t}\right)\right|\right\} \\
& \leq C_{1}\left(e^{|T-t|\|V\|_{\infty}}-1\right) e^{T\|V\|_{\infty}} \mathbb{E}_{\mathfrak{u}} \mathfrak{w}\left(\mathfrak{u}_{t}\right) .
\end{aligned}
$$

Combining this with (5.24), we get $S_{1} \rightarrow 0$ as $t \rightarrow T$. To estimate $S_{2}$, let us take any $R>0$ and write

$$
\begin{aligned}
e^{-T\|V\|_{\infty}\left|S_{2}\right|} & \leq \mathbb{E}_{\mathfrak{u}}\left|g\left(\mathfrak{u}_{T}\right)-g\left(\mathfrak{u}_{t}\right)\right| \\
& =\mathbb{E}_{\mathfrak{u}}\left\{\mathbb{I}_{G_{R}^{c}}\left|g\left(\mathfrak{u}_{T}\right)-g\left(\mathfrak{u}_{t}\right)\right|\right\}+\mathbb{E}_{\mathfrak{u}}\left\{\mathbb{I}_{G_{R}}\left|g\left(\mathfrak{u}_{T}\right)-g\left(\mathfrak{u}_{t}\right)\right|\right\} \\
& =: S_{3}+S_{4},
\end{aligned}
$$

where $G_{R}:=\left\{u_{t}, u_{T} \in X_{R}\right\}$. From the Chebyshev inequality, the fact that $g \in C_{\mathfrak{w}}\left(\mathcal{H}^{r}\right)$, and inequality (5.24) we derive

$$
\begin{aligned}
S_{3} & \leq C_{1} \mathbb{E}_{\mathfrak{u}}\left\{\mathbb{I}_{G_{R}^{c}}\left(\mathfrak{w}\left(\mathfrak{u}_{T}\right)+\mathfrak{w}\left(\mathfrak{u}_{t}\right)\right)\right\} \\
& \leq C_{1} R^{-2} \mathbb{E}_{\mathfrak{u}}\left\{\mathfrak{w}^{2}\left(\mathfrak{u}_{T}\right)+\mathfrak{w}^{2}\left(\mathfrak{u}_{t}\right)\right\} \leq C_{2} R^{-2} \mathfrak{w}^{2}(\mathfrak{u}) .
\end{aligned}
$$


On the other hand, by the Lebesgue theorem on dominated convergence, for any $R>0$, we have $S_{4} \rightarrow 0$ as $t \rightarrow T$. Choosing $R>0$ sufficiently large and $t$ sufficiently close to $T$, we see that $S_{3}+S_{4}$ can be made arbitrarily small. This shows that $S_{2} \rightarrow 0$ as $t \rightarrow T$ and proves the time-continuity property.

Step 2: Application of Theorem A.4. We conclude from Theorem A.4 that there is an eigenvector $\mu_{V} \in \mathcal{P}(\mathcal{H})$ for the semigroup $\mathfrak{P}_{t}^{V *}$ corresponding to some positive eigenvalue $\lambda_{V}$, i.e., $\mathfrak{P}_{t}^{V *} \mu_{V}=\lambda_{V}^{t} \mu_{V}$ for any $t>0$. Moreover, the semigroup $\mathfrak{P}_{t}^{V}$ has an eigenvector $h_{V} \in C_{\mathfrak{w}}\left(\mathcal{H}^{r}\right) \cap C_{+}\left(\mathcal{H}^{r}\right)$ corresponding to $\lambda_{V}$ such that $\left\langle h_{V}, \mu_{V}\right\rangle=1$. The uniqueness of $\mu_{V}$ and $h_{V}$ follows immediately from (1.15) and (1.16). The uniqueness of $\mu_{V}$ implies that it does not depend on $m$ and (1.14) holds for any $m \geq 1$. It remains to prove limits (1.15) and (1.16).

Step 3: Proof of (1.15). By (A.16), we have (1.15) for any $\psi \in \mathcal{U}$. To establish the limit for any $\psi \in C_{\mathfrak{w}}\left(\mathcal{H}^{r}\right)$, we apply an approximation argument similar to the one used in Step 4 of the proof of Theorem 5.5 in [10]. Let us take a sequence $\psi_{n} \in \mathcal{U}$ such that $\left\|\psi_{n}\right\|_{\infty} \leq\|\psi\|_{\infty}$ and $\psi_{n} \rightarrow \psi$ as $n \rightarrow \infty$, uniformly on bounded subsets of $\mathcal{H}^{r}$. If we define

$$
\Delta_{t}(g)=\sup _{\mathfrak{u} \in X_{R}}\left|\lambda_{V}^{-t} \mathfrak{P}_{t}^{V} g(\mathfrak{u})-\left\langle g, \mu_{V}\right\rangle h_{V}(\mathfrak{u})\right|, \quad\|g\|_{R}=\sup _{\mathfrak{u} \in X_{R}}|g(\mathfrak{u})|,
$$

then

$$
\Delta_{t}(\psi) \leq \Delta_{t}\left(\psi_{n}\right)+\left\|h_{V}\right\|_{R}\left|\left\langle\psi-\psi_{n}, \mu_{V}\right\rangle\right|+\lambda_{V}^{-t}\left\|\mathfrak{P}_{t}^{V}\left(\psi-\psi_{n}\right)\right\|_{R}
$$

for any $t \geq 0$ and $n \geq 1$. In view of (1.15) for $\psi_{n}$ and the Lebesgue theorem on dominated convergence,

$$
\begin{aligned}
& \Delta_{t}\left(\psi_{n}\right) \rightarrow 0 \quad \text { as } t \rightarrow \infty \text { for any fixed } n \geq 1, \\
& \left|\left\langle\psi-\psi_{n}, \mu_{V}\right\rangle\right| \rightarrow 0 \quad \text { as } n \rightarrow \infty
\end{aligned}
$$

Thus, it suffices to show that

$$
\sup _{t \geq 0} \lambda_{V}^{-t}\left\|\mathfrak{P}_{t}^{V}\left(\psi-\psi_{n}\right)\right\|_{R} \rightarrow 0 \quad \text { as } n \rightarrow \infty .
$$

To this end, for any $\rho>0$, we write

$$
\left\|\mathfrak{P}_{t}^{V}\left(\psi-\psi_{n}\right)\right\|_{R} \leq J_{1}(t, n, \rho)+J_{2}(t, n, \rho),
$$

where

$$
J_{1}(t, n, \rho)=\left\|\mathfrak{P}_{t}^{V}\left(\left(\psi-\psi_{n}\right) \mathbb{I}_{X_{\rho}}\right)\right\|_{R}, \quad J_{2}(t, n, \rho)=\left\|\mathfrak{P}_{t}^{V}\left(\left(\psi-\psi_{n}\right) \mathbb{I}_{X_{\rho}^{c}}\right)\right\|_{R} .
$$

Since $\psi_{n} \rightarrow \psi$ uniformly on $X_{\rho}$, we have

$$
J_{1}(t, n, \rho) \leq \varepsilon(n, \rho)\left\|\mathfrak{P}_{t}^{V} \mathbf{1}\right\|_{R},
$$

where $\varepsilon(n, \rho) \rightarrow 0$ as $n \rightarrow \infty$. Using convergence (1.15) for $\psi=\mathbf{1}$, we see that

$$
\lambda_{V}^{-t}\left\|\mathfrak{P}_{t}^{V} \mathbf{1}\right\|_{R} \leq C_{3}(R) \quad \text { for all } t \geq 0 .
$$

Hence,

$$
\sup _{t \geq 0} \lambda_{V}^{-t} J_{1}(t, n, \rho) \leq C_{3}(R) \varepsilon(n, \rho) \rightarrow 0 \quad \text { as } n \rightarrow \infty .
$$

We use (3.1) and (6.3), to estimate $J_{2}$ :

$$
\begin{aligned}
\lambda_{V}^{-t} J_{2}(t, n, \rho) & \leq 2\|\psi\|_{\infty} \rho^{-2} \lambda_{V}^{-t}\left\|\mathfrak{P}_{t}^{V} \mathfrak{w}\right\|_{R} \leq C_{4}(R)\|\psi\|_{\infty} \rho^{-2} \lambda_{V}^{-t}\left\|\mathfrak{P}_{t}^{V} \mathbf{1}\right\|_{R_{0}} \\
& \leq C_{4}(R)\|\psi\|_{\infty} \rho^{-2} C_{3}\left(R_{0}\right) .
\end{aligned}
$$


Taking first $\rho$ and then $n$ sufficiently large, we see that $\sup _{t \geq 0} \lambda_{V}^{-t}\left\|\mathfrak{P}_{t}^{V}\left(\psi-\psi_{n}\right)\right\|_{R}$ can be made arbitrarily small. This proves (6.2) and completes the proof of (1.15).

Step 4: Proof of (1.16). Let us show that

$$
\lambda_{V}^{-t}\left\langle\mathfrak{P}_{t}^{V} \psi, v\right\rangle \rightarrow\left\langle\psi, \mu_{V}\right\rangle\left\langle h_{V}, v\right\rangle \quad \text { as } t \rightarrow \infty
$$

for any $\psi \in C_{b}(\mathcal{H})$. In view of (1.15), it suffices to show that

$$
\sup _{t \geq 0}\left\{\int_{H} \mathbb{I}_{X_{R}^{c}}\left|\lambda_{V}^{-t} \mathfrak{P}_{t}^{V} \psi(\mathfrak{u})-\left\langle\psi, \mu_{V}\right\rangle h_{V}(\mathfrak{u})\right| v(\mathrm{~d} \mathfrak{u})\right\} \rightarrow 0 \quad \text { as } R \rightarrow \infty .
$$

From (3.2) and (6.3) we derive that

$$
\left\|\mathfrak{P}_{t}^{V} \psi\right\|_{L_{\mathfrak{w}}^{\infty}} \leq\|\psi\|_{\infty}\left\|\mathfrak{P}_{t}^{V} \mathbf{1}\right\|_{L_{\mathfrak{w}}^{\infty}} \leq C_{5}\left\|\mathfrak{P}_{t}^{V} \mathbf{1}\right\|_{R_{0}} \leq C_{6}\left(R_{0}\right) \lambda_{V}^{t}, \quad t \geq 0
$$

hence

$$
\left|\lambda_{V}^{-k} \mathfrak{P}_{t}^{V} \psi(\mathfrak{u})\right| \leq C_{6}\left(R_{0}\right) \mathfrak{w}(\mathfrak{u}), \quad \mathfrak{u} \in \mathcal{H}^{r}, t \geq 0 .
$$

Since $h_{V} \in C_{\mathfrak{w}}\left(\mathcal{H}^{r}\right)$ and

$$
\int_{\mathcal{H}} \mathbb{I}_{X_{R}^{c}}(\mathfrak{u}) \mathfrak{w}(\mathfrak{u}) v(\mathrm{~d} \mathfrak{u}) \rightarrow 0 \quad \text { as } R \rightarrow \infty
$$

we obtain (6.4). This completes the proof of Theorem 1.2.

\section{Appendix}

\section{A.1. Local version of Kifer's theorem}

In [14], Kifer established a sufficient condition for the validity of the LDP for a family of random probability measures on a compact metric space. This result was extended by Jakšić et al. [10] to the case of a general Polish space. In this section, we obtain a local version of these results. Roughly speaking, we assume the existence of a pressure function (i.e., limit (A.3)) and the uniqueness of the equilibrium state for functions $V$ in a set $\mathcal{V}$, which is not necessarily dense in the space of bounded continuous functions. We prove the LDP with a lower bound in which the infimum of the rate function is taken over a subset of the equilibrium states. To give the exact formulation of the result, we first introduce some notation and definitions. Assume that $X$ is a Polish space, and $\zeta_{\theta}$ is a random probability measure on $X$ defined on some probability space $\left(\Omega_{\theta}, \mathcal{F}_{\theta}, \mathbb{P}_{\theta}\right)$, where the index $\theta$ belongs to some directed $\operatorname{set}^{9} \Theta$ (i.e., a partially ordered set whose every finite subset has an upper bound). Let $r: \Theta \rightarrow \mathbb{R}$ be a positive function such that $\lim _{\theta \in \Theta} r_{\theta}=+\infty$. For any $V \in C_{b}(X)$, let us set

$$
Q(V):=\limsup _{\theta \in \Theta} \frac{1}{r_{\theta}} \log \mathbb{E}_{\theta} \exp \left(r_{\theta}\left\langle V, \zeta_{\theta}\right\rangle\right),
$$

where $\mathbb{E}_{\theta}$ is the expectation with respect to $\mathbb{P}_{\theta}$. The function $Q: C_{b}(X) \rightarrow \mathbb{R}$ is convex, $Q(V) \geq 0$ for any $V \in C_{+}(X)$, and $Q(C)=C$ for any $C \in \mathbb{R}$. Moreover, $Q$ is 1-Lipschitz. Indeed, for any $V_{1}, V_{2} \in C_{b}(X)$ and $\theta \in \Theta$, we have

$$
\frac{1}{r_{\theta}} \log \mathbb{E}_{\theta} \exp \left(r_{\theta}\left\langle V_{1}, \zeta_{\theta}\right\rangle\right) \leq\left\|V_{1}-V_{2}\right\|_{\infty}+\frac{1}{r_{\theta}} \log \mathbb{E}_{\theta} \exp \left(r_{\theta}\left\langle V_{2}, \zeta_{\theta}\right\rangle\right)
$$

\footnotetext{
${ }^{9}$ Let us emphasize that the use of a directed set $\Theta$ to index the family $\zeta_{\theta}$ is necessary, since it allows us to establish the LDP of uniform type with respect to the initial condition (see the proof of Theorem 1.1).
} 
which implies that

$$
Q\left(V_{1}\right) \leq\left\|V_{1}-V_{2}\right\|_{\infty}+Q\left(V_{2}\right)
$$

By symmetry we get

$$
\left|Q\left(V_{1}\right)-Q\left(V_{2}\right)\right| \leq\left\|V_{1}-V_{2}\right\|_{\infty} .
$$

The Legendre transform of $Q$ is given by

$$
I(\sigma)= \begin{cases}\sup _{V \in C_{b}(X)}(\langle V, \sigma\rangle-Q(V)) & \text { for } \sigma \in \mathcal{P}(X), \\ +\infty & \text { for } \sigma \in \mathcal{M}(X) \backslash \mathcal{P}(X)\end{cases}
$$

(see Lemma 2.2 in [1]). Then $I$ is convex and lower semicontinuous function, and

$$
Q(V)=\sup _{\sigma \in \mathcal{P}(X)}(\langle V, \sigma\rangle-I(\sigma)) .
$$

A measure $\sigma_{V} \in \mathcal{P}(X)$ is said to be an equilibrium state for $V$ if

$$
Q(V)=\left\langle V, \sigma_{V}\right\rangle-I\left(\sigma_{V}\right) .
$$

We shall denote by $\mathcal{V}$ the set of functions $V \in C_{b}(X)$ admitting a unique equilibrium state $\sigma_{V}$ and for which the following limit exists

$$
Q(V)=\lim _{\theta \in \Theta} \frac{1}{r_{\theta}} \log \mathbb{E}_{\theta} \exp \left(r_{\theta}\left\langle V, \zeta_{\theta}\right\rangle\right) .
$$

Let us assume that the sequence of functions $\left\{V_{k}\right\} \subset C_{b}(X)$ defines the weak topology in $\mathcal{P}(X)$, and $\mathcal{V}_{1} \subset \mathcal{V}$ is such that the intersection of $\mathcal{V}_{1}$ with $L_{n}:=\operatorname{span}\left\{V_{1}, \ldots, V_{n}\right\}$ is open in $L_{n}$ (endowed with the $\|\cdot\|_{\infty}$-norm). Let $\mathcal{W}_{n}:=\left\{\sigma_{V}: V \in \mathcal{V}_{1} \cap L_{n}\right\}$ and $\mathcal{W}:=\bigcup_{n \geq 1} \mathcal{W}_{n}$

We have the following version of Theorem 2.1 in [14] and Theorem 3.3 in [10].

Theorem A.1. Suppose that there is a function $\Phi: X \rightarrow[0,+\infty]$ whose level sets $\{u \in X: \Phi(u) \leq a\}$ are compact for all $a \geq 0$ and

$$
\mathbb{E}_{\theta} \exp \left(r_{\theta}\left\langle\Phi, \zeta_{\theta}\right\rangle\right) \leq C e^{c r_{\theta}} \quad \text { for } \theta \in \Theta
$$

for some positive constants $C$ and c. Then I defined by (A.2) is a good rate function, for any closed set $F \subset \mathcal{P}(X)$,

$$
\limsup _{\theta \in \Theta} \frac{1}{r_{\theta}} \log \mathbb{P}_{\theta}\left\{\zeta_{\theta} \in F\right\} \leq-I(F)
$$

and for any open set $G \subset \mathcal{P}(X)$,

$$
\liminf _{\theta \in \Theta} \frac{1}{r_{\theta}} \log \mathbb{P}_{\theta}\left\{\zeta_{\theta} \in G\right\} \geq-I(\mathcal{W} \cap G)
$$

where $I(\Gamma):=\inf _{\sigma \in \Gamma} I(\sigma), \Gamma \subset \mathcal{P}(X)$.

Proof. The fact that $I$ is a good rate function is shown in Step 1 of the proof of Theorem 3.3 in [10]. In Step 2 of the same proof, the upper bound (A.5) is established, under the condition that the limit $Q(V)$ in (A.3) exists for any $V \in C_{b}(X)$. The latter condition can be removed, using literally the same proof, if one defines $Q(V)$ by (A.1) for any $V \in C_{b}(X)$ (see Theorem 2.1 in [4]). 
To prove the lower bound, following the ideas of [14], for any $n \geq 1$, we define an auxiliary family of finitedimensional random variables $\zeta_{\theta}^{n}:=f_{n}\left(\zeta_{\theta}\right)$, where $f_{n}: \mathcal{P}(X) \rightarrow \mathbb{R}^{n}$ is given by

$$
f_{n}(\mu):=\left(\left\langle V_{1}, \mu\right\rangle, \ldots,\left\langle V_{n}, \mu\right\rangle\right) .
$$

The following result is a local version of Lemma 2.1 in [14] and Proposition 3.4 in [10]; its proof is sketched at the end of this section.

Proposition A.2. Assume that the hypotheses of Theorem A.1 are satisfied and set $J_{n}(\Gamma)=\inf _{\sigma \in f_{n}^{-1}(\Gamma)} I(\sigma), \Gamma \subset \mathbb{R}^{n}$. Then for any closed set $M \subset \mathbb{R}^{n}$ and open set $U \subset \mathbb{R}^{n}$, we have

$$
\begin{aligned}
& \limsup _{\theta \in \Theta} \frac{1}{r_{\theta}} \log \mathbb{P}\left\{\zeta_{\theta}^{n} \in M\right\} \leq-J_{n}(M), \\
& \liminf _{\theta \in \Theta} \frac{1}{r_{\theta}} \log \mathbb{P}\left\{\zeta_{\theta}^{n} \in U\right\} \geq-J_{n}\left(f_{n}\left(\mathcal{W}_{n}\right) \cap U\right) .
\end{aligned}
$$

To derive (A.6) from Proposition A.2, we follow the arguments of Step 4 of the proof of Theorem 3.3 in [10]. The case $I(\mathcal{W} \cap G)=+\infty$ is trivial, so we assume that $I(\mathcal{W} \cap G)<+\infty$. Then for any $\varepsilon>0$, there is $v_{\varepsilon} \in \mathcal{W} \cap G$ such that

$$
I\left(v_{\varepsilon}\right) \leq I(\mathcal{W} \cap G)+\varepsilon .
$$

By Lemma 3.2 in [10], the family $\left\{\zeta_{\theta}\right\}$ is exponentially tight, hence there is a compact set $\mathcal{K} \subset \mathcal{P}(X)$ such that $v_{\varepsilon} \in \mathcal{K}$ and

$$
\limsup _{\theta \in \Theta} \frac{1}{r_{\theta}} \log \mathbb{P}\left\{\zeta_{\theta} \in \mathcal{K}^{c}\right\} \leq-(I(\mathcal{W} \cap G)+1+\varepsilon) .
$$

Without loss of generality, we can assume that $\left\|V_{k}\right\|_{\infty}=1, k \geq 1$, so that

$$
d(\mu, \nu):=\sum_{k=1}^{\infty} 2^{-k}\left|\left\langle V_{k}, \mu\right\rangle-\left\langle V_{k}, \nu\right\rangle\right|
$$

defines a metric on $\mathcal{K}$ compatible with the weak topology. As $G$ is open, there are $\delta>0$ and $n \geq 1$ such that if

$$
\sum_{k=1}^{n} 2^{-k}\left|\left\langle V_{k}, v\right\rangle-\left\langle V_{k}, v_{\varepsilon}\right\rangle\right|<\delta
$$

for some $v \in \mathcal{K}$, then $v \in G$. We can assume that $n \geq 1$ is so large that $v_{\varepsilon} \in \mathcal{W}_{n}$. Let $x_{\varepsilon}:=f_{n}\left(v_{\varepsilon}\right)$, and denote by $\stackrel{\circ}{B}_{\mathbb{R}^{n}}\left(x_{\varepsilon}, \delta\right)$ the open ball in $\mathbb{R}^{n}$ of radius $\delta>0$ centered at $x_{\varepsilon}$, with respect to the norm

$$
\|x\|_{n}:=\sum_{k=1}^{n} 2^{-k}\left|x_{k}\right|, \quad x=\left(x_{1}, \ldots, x_{n}\right) .
$$

Then we have $f_{n}^{-1}\left(\stackrel{\circ}{B}_{\mathbb{R}^{n}}\left(x_{\varepsilon}, \delta\right)\right) \cap \mathcal{K} \subset G$, hence

$$
\begin{aligned}
\mathbb{P}\left\{\zeta_{\theta} \in G\right\} & \geq \mathbb{P}\left\{\zeta_{\theta} \in G \cap \mathcal{K}\right\} \geq \mathbb{P}\left\{\zeta_{\theta} \in f_{n}^{-1}\left(\stackrel{\circ}{B}_{\mathbb{R}^{n}}\left(x_{\varepsilon}, \delta\right)\right) \cap \mathcal{K}\right\} \\
& \geq \mathbb{P}\left\{\zeta_{\theta}^{n} \in \stackrel{\circ}{B}_{\mathbb{R}^{n}}\left(x_{\varepsilon}, \delta\right)\right\}-\mathbb{P}\left\{\zeta_{\theta} \in \mathcal{K}^{c}\right\} .
\end{aligned}
$$

Using the inequality

$$
\log (u-v) \geq \log u-\log 2, \quad 0<v \leq u / 2
$$


and inequalities (A.8)-(A.10), we obtain

$$
\begin{aligned}
\liminf _{\theta \in \Theta} \frac{1}{r_{\theta}} \log \mathbb{P}\left\{\zeta_{\theta} \in G\right\} & \geq \liminf _{\theta \in \Theta} \frac{1}{r_{\theta}}\left(\log \mathbb{P}\left\{\zeta_{\theta}^{n} \in \stackrel{\circ}{B}_{\mathbb{R}^{n}}\left(x_{\varepsilon}, \delta\right)\right\}-\log 2\right) \\
& \geq-J_{n}\left(f_{n}\left(\mathcal{W}_{n}\right) \cap \stackrel{\circ}{B}_{\mathbb{R}^{n}}\left(x_{\varepsilon}, \delta\right)\right) \geq-J_{n}\left(x_{\varepsilon}\right) \\
& \geq-I\left(v_{\varepsilon}\right) \geq-I(\mathcal{W} \cap G)-\varepsilon,
\end{aligned}
$$

which proves (A.6).

Sketch of the proof of Proposition A.2. Inequality (A.7) follows from (A.5). To show (A.8), for any $\beta=\left(\beta_{1}, \ldots\right.$, $\left.\beta_{n}\right) \in \mathbb{R}^{n}$ and $\alpha=\left(\alpha_{1}, \ldots, \alpha_{n}\right) \in \mathbb{R}^{n}$, we set $V_{\beta}:=\sum_{j=1}^{n} \beta_{j} V_{j}, Q_{n}(\beta):=Q\left(V_{\beta}\right)$, and $I_{n}(\alpha):=\inf _{\sigma \in f_{n}^{-1}(\alpha)} I(\sigma)$. One can verify that

$$
\begin{aligned}
& Q_{n}(\beta)=\sup _{\alpha \in \mathbb{R}^{n}}\left(\sum_{j=1}^{n} \beta_{j} \alpha_{j}-I_{n}(\alpha)\right), \\
& J_{n}(U)=\inf _{\alpha \in U} I_{n}(\alpha) .
\end{aligned}
$$

Assume that $J_{n}\left(f_{n}\left(\mathcal{W}_{n}\right) \cap U\right)<+\infty$, and for any $\varepsilon>0$, choose $\alpha_{\varepsilon} \in f_{n}\left(\mathcal{W}_{n}\right) \cap U$ such that

$$
I_{n}\left(\alpha_{\varepsilon}\right)<J_{n}\left(f_{n}\left(\mathcal{W}_{n}\right) \cap U\right)+\varepsilon .
$$

Then $\alpha_{\varepsilon}=f_{n}\left(\sigma_{V_{\beta_{\varepsilon}}}\right)$ for some $\beta_{\varepsilon} \in \mathbb{R}^{n}$ such that $V_{\beta_{\varepsilon}} \in \mathcal{V}$. It is easy to verify that the following equality holds

$$
Q_{n}\left(\beta_{\varepsilon}\right)=\sum_{j=1}^{n} \beta_{\varepsilon j} \alpha_{\varepsilon j}-I_{n}\left(\alpha_{\varepsilon}\right) .
$$

Literally repeating the proof of Proposition 3.4 in [10] (starting from equality (3.16)) and using the uniqueness of the equilibrium state for $V=V_{\beta_{\varepsilon}}$ and the existence of limit (A.3), one obtains

$$
-J_{n}\left(f_{n}\left(\mathcal{W}_{n}\right) \cap U\right)-\varepsilon \leq-I_{n}\left(\alpha_{\varepsilon}\right) \leq \liminf _{\theta \in \Theta} \frac{1}{r_{\theta}} \log \mathbb{P}\left\{\zeta_{\theta}^{n} \in U\right\}
$$

for any $\varepsilon>0$. This implies (A.8).

\section{A.2. Large-time asymptotics for generalised Markov semigroups}

In this section, we give a continuous-time version of Theorem 4.1 in [10] with some modifications, due to the fact that the generalized Markov family associated with the stochastic NLW equation does not have a regularizing property. See also $[12,17,20]$ for some related results.

We start by recalling some terminology from [10].

Definition A.3. Let $X$ be a Polish space. We shall say that $\left\{P_{t}(\mathfrak{u}, \cdot), \mathfrak{u} \in X, t \geq 0\right\}$ is a generalized Markov family of transition kernels if the following two properties are satisfied.

Feller property. For any $t \geq 0$, the function $\mathfrak{u} \mapsto P_{t}(\mathfrak{u}, \cdot)$ is continuous from $X$ to $\mathcal{M}_{+}(X)$ and does not vanish.

Kolmogorov-Chapman relation. For any $t, s \geq 0, \mathfrak{u} \in X$, and Borel set $\Gamma \subset X$, the following relation holds

$$
P_{t+s}(\mathfrak{u}, \Gamma)=\int_{X} P_{s}(\mathfrak{v}, \Gamma) P_{t}(\mathfrak{u}, \mathrm{d} \mathfrak{v}) .
$$


To any such family we associate two semigroups by the following relations:

$$
\begin{aligned}
& \mathfrak{P}_{t}: C_{b}(X) \rightarrow C_{b}(X), \quad \mathfrak{P}_{t} \psi(\mathfrak{u})=\int_{X} \psi(\mathfrak{v}) P_{t}(\mathfrak{u}, \mathrm{d} \mathfrak{v}), \\
& \mathfrak{P}_{t}^{*}: \mathcal{M}_{+}(X) \rightarrow \mathcal{M}_{+}(X), \quad \mathfrak{P}_{t}^{*} \mu(\Gamma)=\int_{X} P_{t}(\mathfrak{v}, \Gamma) \mu(\mathrm{d} \mathfrak{v}), \quad t \geq 0 .
\end{aligned}
$$

For a measurable function $\mathfrak{w}: X \rightarrow[1,+\infty]$ and a family $\mathcal{C} \subset C_{b}(X)$, we denote by $\mathcal{C}^{\mathfrak{w}}$ the set of functions $\psi \in$ $L_{\mathfrak{w}}^{\infty}(X)$ that can be approximated with respect to $\|\cdot\|_{L_{\mathfrak{w}}^{\infty}}$ by finite linear combinations of functions from $\mathcal{C}$. We shall say that a family $\mathcal{C} \subset C_{b}(X)$ is determining if for any $\mu, v \in \mathcal{M}_{+}(X)$ satisfying $\langle\psi, \mu\rangle=\langle\psi, v\rangle$ for all $\psi \in \mathcal{C}$, we have $\mu=v$. Finally, a family of functions $\psi_{t}: X \rightarrow \mathbb{R}$ is uniformly equicontinuous on a subset $K \subset X$ if for any $\varepsilon>0$ there is $\delta>0$ such that $\left|\psi_{t}(\mathfrak{u})-\psi_{t}(\mathfrak{v})\right|<\varepsilon$ for any $\mathfrak{u} \in K, \mathfrak{v} \in B_{X}(\mathfrak{u}, \delta) \cap K$, and $t \geq 1$. We have the following version of Theorem 4.1 in [10].

Theorem A.4. Let $\left\{P_{t}(\mathfrak{u}, \cdot), \mathfrak{u} \in X, t \geq 0\right\}$ be a generalized Markov family of transition kernels satisfying the following four properties.

Growth conditions. There is an increasing sequence $\left\{X_{R}\right\}_{R=1}^{\infty}$ of compact subsets of $X$ such that $X_{\infty}:=\bigcup_{R=1}^{\infty} X_{R}$ is dense in $X$. The measures $P_{t}(\mathfrak{u}, \cdot)$ are concentrated on $X_{\infty}$ for any $\mathfrak{u} \in X_{\infty}$ and $t>0$, and there is a measurable function $\mathfrak{w}: X \rightarrow[1,+\infty]$ and an integer $R_{0} \geq 1$ such that ${ }^{10}$

$$
\begin{aligned}
& \sup _{t \geq 0} \frac{\left\|\mathfrak{P}_{t} \mathfrak{w}\right\|_{L_{\mathfrak{w}}^{\infty}}}{\left\|\mathfrak{P}_{t} \mathbf{1}\right\|_{R_{0}}}<\infty, \\
& \sup _{t \in[0,1]}\left\|\mathfrak{P}_{t} \mathbf{1}\right\|_{\infty}<\infty,
\end{aligned}
$$

where $\|\cdot\|_{R}$ and $\|\cdot\|_{\infty}$ denote the $L^{\infty}$ norm on $X_{R}$ and $X$, respectively, and we set $\infty / \infty=0$.

Time-continuity. For any function $g \in L_{\mathfrak{w}}^{\infty}\left(X_{\infty}\right)$ whose restriction to $X_{R}$ belongs to $C\left(X_{R}\right)$ and any $\mathfrak{u} \in X_{\infty}$, the function $t \mapsto \mathfrak{P}_{t} g(\mathfrak{u})$ is continuous from $\mathbb{R}_{+}$to $\mathbb{R}$.

Uniform irreducibility. For sufficiently large $\rho \geq 1$, any $R \geq 1$ and $r>0$, there are positive numbers $l=l(\rho, r, R)$ and $p=p(\rho, r)$ such that

$$
P_{l}\left(\mathfrak{u}, B_{X}(\hat{\mathfrak{u}}, r)\right) \geq p \quad \text { for all } \mathfrak{u} \in X_{R}, \hat{\mathfrak{u}} \in X_{\rho} .
$$

Uniform Feller property. There is a number $R_{0} \geq 1$ and a determining family $\mathcal{C} \subset C_{b}(X)$ such that $\mathbf{1} \in \mathcal{C}$ and the family $\left\{\left\|\mathfrak{P}_{t} \mathbf{1}\right\|_{R}^{-1} \mathfrak{P}_{t} \psi, t \geq 1\right\}$ is uniformly equicontinuous on $X_{R}$ for any $\psi \in \mathcal{C}$ and $R \geq R_{0}$.

Then for any $t>0$, there is at most one measure $\mu_{t} \in \mathcal{P}_{\mathfrak{w}}(X)$ such that $\mu_{t}\left(X_{\infty}\right)=1$ and

$$
\mathfrak{P}_{t}^{*} \mu_{t}=\lambda(t) \mu_{t} \quad \text { for some } \lambda(t) \in \mathbb{R}
$$

satisfying the following condition:

$$
\left\|\mathfrak{P}_{t} \mathfrak{w}\right\|_{R} \int_{X \backslash X_{R}} \mathfrak{w} \mathrm{d} \mu_{t} \rightarrow 0 \quad \text { as } R \rightarrow \infty .
$$

Moreover, if such a measure $\mu_{t}$ exists for all $t>0$, then it is independent of $t$ (we set $\mu:=\mu_{t}$ ), the corresponding eigenvalue is of the form $\lambda(t)=\lambda^{t}, \lambda>0$, supp $\mu=X$, and there is a non-negative function $h \in L_{\mathfrak{w}}^{\infty}\left(X_{\infty}\right)$ such that $\langle h, \mu\rangle=1$,

$$
\left(\mathfrak{P}_{t} h\right)(\mathfrak{u})=\lambda^{t} h(\mathfrak{u}) \quad \text { for } \mathfrak{u} \in X_{\infty}, t>0,
$$

\footnotetext{
${ }^{10}$ The expression $\left(\mathfrak{P}_{t} \mathfrak{w}\right)(\mathfrak{u})$ is understood as an integral of a positive function $\mathfrak{w}$ against a positive measure $P_{t}(\mathfrak{u}, \cdot)$.
} 
the restriction of $h$ to $X_{R}$ belongs to $C_{+}\left(X_{R}\right)$, and for any $\psi \in \mathcal{C}^{\mathfrak{w}}$ and $R \geq 1$, we have

$$
\lambda^{-t} \mathfrak{P}_{t} \psi \rightarrow\langle\psi, \mu\rangle h \quad \text { in } C\left(X_{R}\right) \cap L^{1}(X, \mu) \text { as } t \rightarrow \infty .
$$

Finally, if a Borel set $B \subset X$ is such that

$$
\sup _{\mathfrak{u} \in B}\left(\int_{X \backslash X_{R}} \mathfrak{w}(\mathfrak{v}) P_{S}(\mathfrak{u}, \mathrm{d} \mathfrak{v})\right) \rightarrow 0 \quad \text { as } R \rightarrow \infty
$$

for some $s>0$, then for any $\psi \in \mathcal{C}^{\mathfrak{w}}$, we have

$$
\lambda^{-t} \mathfrak{P}_{t} \psi \rightarrow\langle\psi, \mu\rangle h \quad \text { in } L^{\infty}(B) \text { as } t \rightarrow \infty .
$$

Sketch of the proof. Step 1: Existence of eigenvectors $\mu$ and $h$. For any $t>0$, the conditions of Theorem 4.1 in [10] are satisfied ${ }^{11}$ for the discrete-time semigroup $\left\{\tilde{\mathfrak{P}}_{k}=\mathfrak{P}_{t k}, k \geq 1\right\}$ generated by $\tilde{P}=P_{t}$. So that theorem implies the existence of at most one measure $\mu_{t} \in \mathcal{P}_{\mathfrak{w}}(X)$ satisfying $\mu_{t}\left(X_{\infty}\right)=1$, (A.13), and (A.14). Moreover, if such a measure $\mu_{t}$ exists for any $t>0$, it follows from the Kolmogorov-Chapman relation that $\mu_{t}=\mu_{1}=: \mu$ and $\lambda(t)=(\lambda(1))^{t}=: \lambda^{t}$ for any $t$ in the set $\mathbb{Q}_{+}^{*}$ of positive rational numbers, i.e.,

$$
\mathfrak{P}_{t}^{*} \mu=\lambda^{t} \mu \quad \text { for } t \in \mathbb{Q}_{+}^{*} .
$$

Using the time-continuity property and density, we get that (A.20) holds for any $t>0$. So we have $\mu_{t}=\mu$ and $\lambda(t)=\lambda^{t}$ for any $t>0$, by uniqueness of the eigenvector.

Theorem 4.1 in [10] also implies that $\operatorname{supp} \mu=X, \lambda>0$, and there is a non-negative function $h_{t} \in L_{\mathfrak{w}}^{\infty}\left(X_{\infty}\right)$ such that $\left\langle h_{t}, \mu\right\rangle=1$, the restriction of $h_{t}$ to $X_{R}$ belongs to $C_{+}\left(X_{R}\right)$, and

$$
\begin{aligned}
& \left(\mathfrak{P}_{t} h_{t}\right)(\mathfrak{u})=\lambda^{t} h_{t}(\mathfrak{u}) \quad \text { for } \mathfrak{u} \in X_{\infty}, \\
& \lambda^{-t k} \mathfrak{P}_{t k} \psi \rightarrow\langle\psi, \mu\rangle h_{t} \quad \text { in } C\left(X_{R}\right) \cap L^{1}(X, \mu) \text { as } k \rightarrow \infty
\end{aligned}
$$

for any $\psi \in \mathcal{C}^{\mathfrak{w}}, R \geq 1$, and $t>0$. Taking $\psi=\mathbf{1}$ in (A.22), we see that $h_{t}=h_{1}=: h$ for any $t \in \mathbb{Q}_{+}^{*}$. The continuity of the function $t \mapsto \mathfrak{P}_{t} h(\mathfrak{u})$ and (A.21) imply that $h_{t}=h$ for any $t>0$ and

$$
\lambda^{-t k} \mathfrak{P}_{t k} \psi \rightarrow\langle\psi, \mu\rangle h \quad \text { in } C\left(X_{R}\right) \cap L^{1}(X, \mu) \text { as } k \rightarrow \infty .
$$

Step 2: Proof of (A.16). First let us prove (A.16) for any $\psi \in \mathcal{C}$. Replacing $P_{t}(\mathfrak{u}, \Gamma)$ by $\lambda^{-t} P_{t}(\mathfrak{u}, \Gamma)$, we may assume that $\lambda=1$. Taking $\psi=\mathbf{1}$ and $t=1$ in (A.23), we obtain $\sup _{k \geq 0}\left\|\mathfrak{P}_{k} \mathbf{1}\right\|_{R}<\infty$. So using (A.12), we get $\sup _{t \geq 0}\left\|\mathfrak{P}_{t} \mathbf{1}\right\|_{R}<\infty$. This implies that $\left\{\mathfrak{P}_{t} \psi, t \geq 1\right\}$ is uniformly equicontinuous on $X_{R}$ for any $R \geq R_{0}$. Setting $g=$ $\psi-\langle\psi, \mu\rangle h$, we need to prove that $\mathfrak{P}_{t} g \rightarrow 0$ in $C\left(X_{R}\right)$ for any $R \geq 1$. Since $\left\{\mathfrak{P}_{t} g, t \geq 1\right\}$ is uniformly equicontinuous on $X_{R}$, the required assertion will be established if we prove that

$$
\left|\mathfrak{P}_{t} g\right|_{\mu}:=\left\langle\left|\mathfrak{P}_{t} g\right|, \mu\right\rangle \rightarrow 0 \quad \text { as } t \rightarrow \infty .
$$

For any $\varphi \in L_{\mathfrak{w}}^{\infty}(X)$, we have

$$
\left|\mathfrak{P}_{t} \varphi\right|_{\mu} \leq\left|\mathfrak{P}_{t}\right| \varphi|, \mu\rangle=\langle|\varphi|, \mu\rangle=|\varphi|_{\mu},
$$

\footnotetext{
${ }^{11}$ Let us note that in Theorem 4.1 in [10] it is assumed that the measures $P_{t}(\mathfrak{u}, \cdot)$ are concentrated on $X_{\infty}$ for any $\mathfrak{u} \in X$. Here this is replaced by the condition that the measures $P_{t}(\mathfrak{u}, \cdot)$ and $\mu_{t}$ are concentrated on $X_{\infty}$ for any $u \in X_{\infty}$. The uniform irreducibility property is slightly different from the one assumed in [10]. Both modifications are due to the lack of a regularizing property for the stochastic NLW equation. These changes do not affect the proof given in [10], one only needs to replace inequality (4.16) in the proof by the inequality$$
\sup _{k \geq 0}\left\|\mathfrak{P}_{k} \psi\right\|_{L_{\mathfrak{w}}^{\infty}(X)} \leq M_{1}\|\psi\|_{L_{\mathfrak{w}}^{\infty}(X)} \quad \text { for any } \psi \in L_{\mathfrak{w}}^{\infty}(X),
$$

and literally repeat all the arguments. The proof of (A.19) is similar to the one of (4.16). Under these modified conditions, the concept of eigenfunction for $\mathfrak{P}_{t}$ is understood in a weaker sense; namely, relation (A.15) needs to hold only for $\mathfrak{u} \in X_{\infty}$.
} 
thus $\left|\mathfrak{P}_{t} g\right|_{\mu}$ is a non-increasing function in $t$. By (A.23), we have $\left|\mathfrak{P}_{t k} g\right|_{\mu} \rightarrow 0$ as $k \rightarrow \infty$. This proves (A.24), hence also (A.16) for any $\psi \in \mathcal{C}$.

An easy approximation argument shows that (A.16) holds for any $\psi \in \mathcal{C}^{\mathfrak{w}}$ (see Step 4 of the proof of Theorem 4.1 in [10]). Finally, the proof of (A.18) under condition (A.17) is exactly the same as in Step 7 of the proof of the discrete-time case.

\section{A.3. Proofs of some auxiliary assertions}

The Foiaş-Prodi estimate

Here we briefly recall an a priori estimate established in Proposition 4.1 in [21]. Let $\mathfrak{u}_{t}=[u, \dot{u}]$ and $\mathfrak{v}_{t}=[v, \dot{v}]$ be some flows of the equations

$$
\begin{aligned}
& \partial_{t}^{2} u+\gamma \partial_{t} u-\Delta u+f(u)=h(x)+\partial_{t} \varphi(t, x), \\
& \partial_{t}^{2} v+\gamma \partial_{t} v-\Delta v+f(v)+\mathrm{P}_{N}[f(u)-f(v)]=h(x)+\partial_{t} \varphi(t, x),
\end{aligned}
$$

where $\varphi$ is a function belonging to $L_{l o c}^{2}\left(\mathbb{R}_{+}, L^{2}(D)\right)$. We recall that $\mathrm{P}_{N}$ stands for the orthogonal projection in $L^{2}(D)$ onto the vector span $H_{N}$ of the functions $e_{1}, e_{2}, \ldots, e_{N}$ and $P_{N}$ is the projection in $\mathcal{H}$ onto $\mathcal{H}_{N}:=H_{N} \times H_{N}$.

Proposition A.5. Assume that, for some non-negative numbers $s$ and $T$, we have $\mathfrak{u}, \mathfrak{v} \in C(s, s+T ; \mathcal{H})$. Then

$$
\left|P_{N}\left(\mathfrak{v}_{t}-\mathfrak{u}_{t}\right)\right|_{\mathcal{H}}^{2} \leq e^{-\alpha(t-s)}\left|\mathfrak{v}_{s}-\mathfrak{u}_{s}\right|_{\mathcal{H}}^{2} \quad \text { for } s \leq t \leq s+T,
$$

where $\alpha>0$ is the constant entering (1.4). If we suppose that the inequality holds

$$
\int_{s}^{t}\|\nabla z\|^{2} d \tau \leq l+K(t-s) \quad \text { for } s \leq t \leq s+T
$$

for $z=u$ and $z=v$ and some positive numbers $K$ and $l$, then, for any $\varepsilon>0$, there is an integer $N_{*}=N_{*}(\varepsilon, K) \geq 1$ such that

$$
\left|\mathfrak{v}_{t}-\mathfrak{u}_{t}\right|_{\mathcal{H}}^{2} \leq e^{-\alpha(t-s)+\varepsilon l}\left|\mathfrak{v}_{s}-\mathfrak{u}_{s}\right|_{\mathcal{H}}^{2} \quad \text { for } s \leq t \leq s+T
$$

for all $N \geq N_{*}$ and $s \leq t \leq s+T$.

Proof. Estimate (A.29) is proved in Proposition 4.1 in [21]. To prove (A.27), let us note that $\mathfrak{z}=[z, \dot{z}]=P_{N}(\mathfrak{v}-\mathfrak{u}$ ) is a solution of the linear equation

$$
\partial_{t}^{2} z+\gamma \partial_{t} z-\Delta z=0
$$

So we have

$$
\left|P_{N}\left(\mathfrak{v}_{t}-\mathfrak{u}_{t}\right)\right|_{\mathcal{H}}^{2}=\left|\mathfrak{z}_{t}\right|_{\mathcal{H}}^{2} \leq e^{-\alpha(t-s)}\left|\mathfrak{z}_{s}\right|_{\mathcal{H}}^{2} \leq e^{-\alpha(t-s)}\left|\mathfrak{v}_{s}-\mathfrak{u}_{s}\right|_{\mathcal{H}}^{2}
$$

Proof of Proposition 4.1

This proposition is essentially proved in Section 4.2 in [21] in a different form. However, since it plays a central role in the proof of the uniform Feller property, we find it worthwhile to give here a detailed proof of it. As in [21], we follow the arguments presented in Section 3.3 of [19] and Section 4 of [16]. As inequality (4.2) concerns only the laws of solutions, we can assume that the underlying probability space $(\Omega, \mathcal{F}, \mathbb{P})$ is of a particular form. We assume that $\Omega=C\left(\mathbb{R}_{+}, \mathbb{R}\right)$ is endowed with the topology of uniform convergence on bounded intervals, $\mathbb{P}$ is the law of the Wiener process $\hat{\xi}=[0, \xi]$, where $\xi$ is defined in $(0.3)$, and $\mathcal{F}$ is the completion of the Borel $\sigma$-algebra of $\Omega$ with respect to $\mathbb{P}$.

We introduce some notation. Let $\hat{\mathcal{H}}_{N}$ be the $N$-dimensional subspace of $\mathcal{H}$ spanned by the vectors $\hat{e}_{1}, \hat{e}_{2}, \ldots, \hat{e}_{N}$, where $\hat{e}_{j}=\left[0, e_{j}\right]$. Then $\Omega=\Omega_{N} \dot{+} \Omega_{N}^{\perp}$, where $\Omega_{N}=C\left(\mathbb{R}_{+}, \hat{\mathcal{H}}_{N}\right)$ and $\Omega_{N}^{\perp}=C\left(\mathbb{R}_{+}, \hat{\mathcal{H}}_{N}^{\perp}\right)$. For $\omega=\omega_{1} \dot{+} \omega_{2}$, we write 
$\omega=\left(\omega_{1}, \omega_{2}\right)$. For any continuous process $\mathfrak{u}_{t}$ with range in $\mathcal{H}$, we introduce the functional

$$
\mathcal{F}^{\mathfrak{u}}(t)=\left|\mathcal{E}\left(\mathfrak{u}_{t}\right)\right|+\alpha \int_{0}^{t}\left|\mathcal{E}\left(\mathfrak{u}_{s}\right)\right| \mathrm{d} s
$$

and the stopping time

$$
\tau^{\mathfrak{u}}=\inf \left\{t \geq 0: \mathcal{F}^{\mathfrak{u}}(t) \geq \mathcal{F}^{\mathfrak{u}}(0)+L t+\rho\right\},
$$

where $L$ and $\rho$ are some positive constants to be chosen later. Now let us fix initial points $\mathfrak{z}$ and $\mathfrak{z}^{\prime}$ in $\mathcal{H}$. We shall denote by $\mathfrak{u}_{t}$ and $\mathfrak{u}_{t}^{\prime}$ the flows of $(0.1)$ issued from $\mathfrak{z}$ and $\mathfrak{z}^{\prime}$, respectively, and by $\mathfrak{v}(t)$ the flow of (4.1). We define a stopping time $\tilde{\tau}=\tau^{\mathfrak{u}} \wedge \tau^{\mathfrak{u}^{\prime}} \wedge \tau^{\mathfrak{v}}$ and a transformation $\Lambda: \Omega \rightarrow \Omega$ given by

$$
\Lambda(\omega)(t)=\omega(t)-\int_{0}^{t} \varphi(s) \mathrm{d} s, \quad \varphi(t)=\mathbb{1}_{t \leq \tilde{\tau}} \hat{P}_{N}\left(0,\left[f\left(u_{t}\right)-f\left(v_{t}\right)\right]\right),
$$

where $\mathbb{1}_{t \leq \tilde{\tau}}$ stands for the indicator function of the interval $[0, \tilde{\tau}], \hat{P}_{N}$ is the orthogonal projection in $\mathcal{H}$ onto $\hat{\mathcal{H}}_{N}$, and $u$ is the first component of $\mathfrak{u}$. Let us prove the following result, which is a global version of Lemma 4.3 in [21].

Lemma A.6. For any initial points $\mathfrak{z}$ and $\mathfrak{z}^{\prime}$ in $\mathcal{H}$, we have

$$
\left|\Lambda_{*} \mathbb{P}-\mathbb{P}\right|_{\text {var }} \leq\left[\exp \left(C_{N}\left|\mathfrak{z}-\mathfrak{z}^{\prime}\right|_{\mathcal{H}}^{2} e^{\left(|\mathcal{E}(\mathfrak{z})|+\left|\mathcal{E}\left(\mathfrak{z}^{\prime}\right)\right|\right)+\rho}\right)-1\right]^{1 / 2},
$$

where $\Lambda_{*} \mathbb{P}$ stands for the image of $\mathbb{P}$ under $\Lambda$.

Proof of Lemma A.6. Step 1. By the definition of $\tilde{\tau}$, we have

$$
\mathcal{F}^{\mathfrak{u}}(t) \leq \mathcal{F}^{\mathfrak{u}}(0)+L t+\rho, \quad \mathcal{F}^{\mathfrak{v}}(t) \leq \mathcal{F}^{\mathfrak{u}^{\prime}}(0)+L t+\rho
$$

for all $t \leq \tilde{\tau}$. Let us show that there is an integer $N_{1}=N_{1}(L)$ such that, for all $N \geq N_{1}$ and $t \leq \tilde{\tau}$, we have

$$
|\mathfrak{v}(t)-\mathfrak{u}(t)|_{\mathcal{H}}^{2} \leq e^{-\alpha t+\theta}\left|\mathfrak{z}^{\prime}-\mathfrak{z}\right|_{\mathcal{H}}^{2}, \quad \theta=\frac{|\mathcal{E}(\mathfrak{z})| \vee\left|\mathcal{E}\left(\mathfrak{z}^{\prime}\right)\right|+\rho}{2} .
$$

Indeed, thanks to (1.2) and the Poincaré inequality, we have

$$
\begin{aligned}
|\mathfrak{u}|_{\mathcal{H}}^{2} & \leq\left.|| \mathfrak{u}\right|_{\mathcal{H}} ^{2}+2 \int_{D} F\left(u_{1}\right) \mathrm{d} x\left|-2 \int_{D} F\left(u_{1}\right) \mathrm{d} x \leq\right| \mathcal{E}(\mathfrak{u}) \mid+2 v\left\|u_{1}\right\|^{2}+2 C \\
& \leq|\mathcal{E}(\mathfrak{u})|+\frac{\lambda_{1}}{4}\left\|u_{1}\right\|^{2}+2 C \leq|\mathcal{E}(\mathfrak{u})|+\frac{1}{4}|\mathfrak{u}|_{\mathcal{H}}^{2}+2 C,
\end{aligned}
$$

for any $\mathfrak{u}=\left[u_{1}, u_{2}\right]$ in $\mathcal{H}$. Therefore

$$
|\mathfrak{u}|_{\mathcal{H}}^{2} \leq 2|\mathcal{E}(\mathfrak{u})|+3 C .
$$

Combining this inequality with (A.31), we see that for all $t \leq \tilde{\tau}$

$$
\alpha \int_{0}^{t}\|\nabla w(s)\|^{2} \mathrm{~d} s \leq 2\left(|\mathcal{E}(\mathfrak{z})| \vee\left|\mathcal{E}\left(\mathfrak{z}^{\prime}\right)\right|+\rho\right)+2(L+3 C) t
$$

for $w=u$ and $w=v$. Using the above inequality and applying Proposition A.5 with $\varepsilon=\alpha / 4$, we infer (A.32).

Step 2. Note that the transformation $\Lambda$ can be written as $\Lambda(\omega)=\left(\Upsilon(\omega), \omega_{2}\right)$, where $\Upsilon: \Omega \rightarrow \Omega_{N}$ is given by

$$
\Upsilon(\omega)(t)=\omega_{1}(t)+\int_{0}^{t} \varphi(s ; \omega) \mathrm{d} s .
$$


It is not difficult to see that

$$
\left|\Lambda_{*} \mathbb{P}-\mathbb{P}\right|_{\mathrm{var}} \leq \int_{\Omega_{N}^{\perp}}\left|\Upsilon_{*}\left(\mathbb{P}_{N}, \omega_{2}\right)-\mathbb{P}_{N}\right|_{\mathrm{var}} \mathbb{P}_{N}^{\perp}\left(\mathrm{d} \omega_{2}\right),
$$

where $\mathbb{P}_{N}$ and $\mathbb{P}_{N}^{\perp}$ stand for the images of $\mathbb{P}$ under the projections $\hat{P}_{N}: \Omega \rightarrow \Omega_{N}$ and $\hat{Q}_{N}: \Omega \rightarrow \Omega_{N}^{\perp}$, respectively. Introduce

$$
X=\omega_{1}(t), \quad \hat{X}=\omega_{1}(t)+\int_{0}^{t} \varphi(s ; \omega) \mathrm{d} s .
$$

Then $\mathbb{P}_{N}$ coincides with the distribution $\mathcal{D}(X)$ of the random variable $X$ and $\Upsilon_{*}\left(\mathbb{P}_{N}, \omega_{2}\right)$ coincides with that of $\hat{X}$. By the Girsanov theorem (see Theorem A.10.1 in [19]), we have

$$
|\mathcal{D}(\hat{X})-\mathcal{D}(X)|_{\mathrm{var}} \leq \frac{1}{2}\left(\left(\mathbb{E} \exp \left[6 \max _{1 \leq j \leq N} b_{j}^{-1} \int_{0}^{\infty}|\varphi(t)|^{2} \mathrm{~d} t\right]\right)^{\frac{1}{2}}-1\right)^{\frac{1}{2}},
$$

if we assume that the Novikov condition

$$
\mathbb{E} \exp \left(c \int_{0}^{\infty}|\varphi(t)|^{2} \mathrm{~d} t\right)<\infty \text { for any } c>0,
$$

is satisfied. To check this condition, first note that

$$
\begin{aligned}
\mathbb{E} \exp \left(c \int_{0}^{\infty}|\varphi(t)|^{2} \mathrm{~d} t\right) & =\mathbb{E} \exp \left(c \int_{0}^{\tilde{\tau}}|\varphi(t)|^{2} \mathrm{~d} t\right) \\
& \leq \mathbb{E} \exp \left(c \int_{0}^{\tilde{\tau}}\left\|f\left(v_{t}\right)-f\left(u_{t}\right)\right\|^{2} \mathrm{~d} t\right) .
\end{aligned}
$$

Using (1.1), the Hölder inequality, and the Sobolev embedding $H^{1} \hookrightarrow L^{6}$, we see that

$$
\|f(v)-f(u)\|^{2} \leq C_{1}\|v-u\|_{1}^{2}\left(1+\|u\|_{1}^{4}+\|v\|_{1}^{4}\right) .
$$

Joining this together with inequalities (A.31)-(A.33) and (A.36), we get

$$
\begin{aligned}
& \mathbb{E} \exp \left(c \int_{0}^{\infty}|\varphi(t)|^{2} \mathrm{~d} t\right) \\
& \quad \leq \mathbb{E} \exp \left(C_{2}\left|\mathfrak{z}^{\prime}-\mathfrak{z}\right|_{\mathcal{H}}^{2} \int_{0}^{\infty} e^{-\alpha t+\theta}\left(1+|\mathcal{E}(\mathfrak{z})| \vee\left|\mathcal{E}\left(\mathfrak{z}^{\prime}\right)\right|+L t+\rho\right)^{2} \mathrm{~d} t\right) \\
& \quad \leq \exp \left(C_{3}\left|\mathfrak{z}-\mathfrak{z}^{\prime}\right|_{\mathcal{H}}^{2} e^{\left(|\mathcal{E}(\mathfrak{z})|+\left|\mathcal{E}\left(\mathfrak{z}^{\prime}\right)\right|\right)+\rho}\right)<\infty .
\end{aligned}
$$

Finally, combining this with (A.34) and (A.35), we get (A.30).

Now we can prove (4.2). Indeed, for each $\omega$ belonging to the event $\{\tilde{\tau}<\infty\}$, let us introduce auxiliary $\mathcal{H}$ continuous processes $y_{\mathfrak{u}^{\prime}}$ and $y_{\mathfrak{v}}$ defined as follows: for $t \leq \tilde{\tau}$ they coincide with the processes $\mathfrak{u}^{\prime}$ and $\mathfrak{v}$, respectively, while for $t \geq \tilde{\tau}$ they solve $\dot{y}=-m y$. We choose $m \geq 1$ so large that

$$
\left\{\tau^{y_{\mathfrak{u}^{\prime}}}<\infty\right\} \subset\left\{\tau^{\mathfrak{u}^{\prime}}<\infty\right\}
$$

This construction implies that, with probability 1 , we have

$$
y_{\mathfrak{v}}(t, \omega)=y_{\mathfrak{u}^{\prime}}(t, \Lambda(\omega)) \text { for all } t \geq 0 .
$$


Let us denote by $\mathfrak{u}_{1}^{\prime}$ and $\mathfrak{v}_{1}$ the restrictions of $\mathfrak{u}^{\prime}(t)$ and $\mathfrak{v}(t)$ to the time interval $[0,1]$. Then

$$
\begin{aligned}
\left|\lambda\left(\mathfrak{z}, \mathfrak{z}^{\prime}\right)-\lambda\left(\mathfrak{z}^{\prime}\right)\right|_{\mathrm{var}} & =\sup _{\Gamma}\left|\mathbb{P}\left\{\mathfrak{v}_{1} \in \Gamma\right\}-\mathbb{P}\left\{\mathfrak{u}_{1}^{\prime} \in \Gamma\right\}\right| \\
& \leq \mathbb{P}\{\tilde{\tau}<\infty\}+\sup _{\Gamma}\left|\mathbb{P}\left\{\mathfrak{v}_{1} \in \Gamma, \tilde{\tau}=\infty\right\}-\mathbb{P}\left\{\mathfrak{u}_{1}^{\prime} \in \Gamma, \tilde{\tau}=\infty\right\}\right|=\mathcal{L}_{1}+\mathcal{L}_{2},
\end{aligned}
$$

where the supremum is taken over all Borel subsets of $C(0,1 ; \mathcal{H})$. Note that

$$
\mathcal{L}_{2} \leq\left|\Lambda_{*} \mathbb{P}-\mathbb{P}\right|_{\text {var }}
$$

Further, we have

$$
\mathcal{L}_{1} \leq \mathbb{P}\left\{\tau^{\mathfrak{v}}<\infty, \tau^{\mathfrak{u}} \wedge \tau^{\mathfrak{u}^{\prime}}=\infty\right\}+\mathbb{P}\left\{\tau^{\mathfrak{u}}<\infty\right\}+\mathbb{P}\left\{\tau^{\mathfrak{u}^{\prime}}<\infty\right\}
$$

Moreover, thanks to (A.38) and (A.37), we have

$$
\begin{aligned}
\mathbb{P}\left\{\tau^{\mathfrak{v}}<\infty, \tau^{\mathfrak{u}} \wedge \tau^{\mathfrak{u}^{\prime}}=\infty\right\} & \leq \mathbb{P}\left\{\tau^{y_{\mathfrak{v}}}<\infty\right\}=\Lambda_{*} \mathbb{P}\left\{\tau^{y_{\mathfrak{u}^{\prime}}}<\infty\right\} \\
& \leq \mathbb{P}\left\{\tau^{y_{\mathfrak{u}^{\prime}}}<\infty\right\}+\left|\Lambda_{*} \mathbb{P}-\mathbb{P}\right|_{\text {var }} \\
& \leq \mathbb{P}\left\{\tau^{\mathfrak{u}^{\prime}}<\infty\right\}+\left|\Lambda_{*} \mathbb{P}-\mathbb{P}\right|_{\text {var }} .
\end{aligned}
$$

Combining last four inequalities, we infer

$$
\left|\lambda\left(\mathfrak{z}, \mathfrak{z}^{\prime}\right)-\lambda\left(\mathfrak{z}^{\prime}\right)\right|_{\text {var }} \leq 2\left(\mathbb{P}\left\{\tau^{\mathfrak{u}}<\infty\right\}+\mathbb{P}\left\{\tau^{u^{\prime}}<\infty\right\}+\left|\Lambda_{*} \mathbb{P}-\mathbb{P}\right|_{\text {var }}\right) .
$$

Finally using this with inequality (A.30) and Corollary 3.3 from [21], we get

$$
\left|\lambda\left(\mathfrak{z}, \mathfrak{z}^{\prime}\right)-\lambda\left(\mathfrak{z}^{\prime}\right)\right|_{\mathrm{var}} \leq 2 e^{4 \beta C-\beta \rho}+2\left[\exp \left(C_{N}\left|\mathfrak{z}-\mathfrak{z}^{\prime}\right|_{\mathcal{H}}^{2} e^{\left(|\mathcal{E}(\mathfrak{z})|+\left|\mathcal{E}\left(\mathfrak{z}^{\prime}\right)\right|\right)+\rho}\right)-1\right]^{1 / 2},
$$

where $\beta=\alpha / 8\left(\sup b_{j}^{2}\right)^{-1}$ and $C$ is the constant entering inequalities (1.1)-(1.3). Denoting $a=2 \beta /(\beta+1)$ and $C_{*}=2 \exp (4 \beta C)$, and making a change of variable $\rho=-\beta^{-1} a \ln \varepsilon$, we derive (4.2).

\section{Proof of Proposition 1.3}

Step 1: Preliminaries. We denote by $\mathfrak{S}_{t}^{V, F}$ the semigroup defined by (1.17), and write $\mathfrak{S}_{t}^{V}$ instead of $\mathfrak{S}_{t}^{V, 0}$ (i.e., $F=\mathbf{0})$. Let $\mathfrak{D}\left(\mathbb{L}_{V}\right)$ be the space of functions $\psi \in C_{b}\left(\mathcal{H}^{\mathfrak{s}}\right)$ such that

$$
\mathfrak{S}_{t}^{V} \psi(\mathfrak{u})=\psi(\mathfrak{u})+\int_{0}^{t} \mathfrak{S}_{\tau}^{V} g(\mathfrak{u}) \mathrm{d} \tau, \quad t \geq 0, \mathfrak{u} \in \mathcal{H}^{r}
$$

for some $g \in C_{b}\left(\mathcal{H}^{r}\right)$. Then the continuity of the mapping $t \mapsto \mathfrak{S}_{t}^{V} g(\mathfrak{u})$ from $\mathbb{R}_{+}$to $\mathbb{R}$ implies the following limit

$$
g(\mathfrak{u})=\lim _{t \rightarrow 0} \frac{\mathfrak{S}_{t}^{V} \psi(\mathfrak{u})-\psi(\mathfrak{u})}{t},
$$

and proves the uniqueness of $g$ in representation (A.39). We set $\mathbb{L}_{V} \psi:=g$. The proof is based on the following two lemmas.

Lemma A.7. For any $F \in C_{b}\left(\mathcal{H}^{r}\right)$, the following properties hold

(i) For any $\psi \in \mathfrak{D}\left(\mathbb{L}_{V}\right)$, we have $\varphi_{t}:=\mathfrak{S}_{t}^{V, F} \psi \in \mathfrak{D}\left(\mathbb{L}_{V}\right)$ and

$$
\partial_{t} \varphi_{t}=\left(\mathbb{L}_{V}+F\right) \varphi_{t}, \quad t>0 .
$$

(ii) The set $\mathfrak{D}_{+}:=\left\{\psi \in \mathfrak{D}\left(\mathbb{L}_{V}\right): \inf _{\mathfrak{u} \in \mathcal{H}^{r}} \psi(\mathfrak{u})>0\right\}$ is determining for $\mathcal{P}\left(\mathcal{H}^{r}\right)$, i.e., if $\left\langle\psi, \sigma_{1}\right\rangle=\left\langle\psi, \sigma_{2}\right\rangle$ for some $\sigma_{1}, \sigma_{2} \in \mathcal{P}\left(\mathcal{H}^{r}\right)$ and any $\psi \in \mathfrak{D}_{+}$, then $\sigma_{1}=\sigma_{2}$. 
This lemma is proved at the end of this subsection. The next result is established exactly in the same way as Lemma 5.9 in [10], by using limit (1.15); we omit its proof.

Lemma A.8. The Markov semigroup $\mathfrak{S}_{t}^{V}$ has a unique stationary measure, which is given by $v_{V}=h_{V} \mu_{V}$.

Step 2. Let us show that, for any $\psi \in \mathfrak{D}_{+}$, we have

$$
Q_{R}^{V}\left(F_{\psi}\right)=0
$$

where $F_{\psi}:=-\mathbb{L}_{V} \psi / \psi \in C_{b}\left(\mathcal{H}^{s}\right)$ and $Q_{R}^{V}\left(F_{\psi}\right)$ is defined by (1.18). Indeed, by property (i) in Lemma A.7, the function $\varphi_{t}=\mathfrak{S}_{t}^{V, F_{\psi}} \psi$ satisfies

$$
\partial_{t} \varphi_{t}=\left(\mathbb{L}_{V}-\frac{\mathbb{L}_{V} \psi}{\psi}\right) \varphi_{t}, \quad \varphi_{0}=\psi
$$

From the uniqueness of the solution we derive that $\psi=\varphi_{t}$ for any $t \geq 0$, hence

$$
\lim _{t \rightarrow+\infty} \frac{1}{t} \log \sup _{\mathfrak{u} \in X_{R}} \log \left(\mathfrak{S}_{t}^{V, F_{\psi}} \psi\right)(\mathfrak{u})=0 .
$$

As $c \leq \psi(\mathfrak{u}) \leq C$ for any $\mathfrak{u} \in \mathcal{H}^{r}$ and some constants $C, c>0$, we have

$$
Q_{R}^{V}\left(F_{\psi}\right) \leq \limsup _{t \rightarrow+\infty} \frac{1}{t} \log \sup _{\mathfrak{u} \in X_{R}} \log \left(\mathfrak{S}_{t}^{V, F_{\psi}} \psi\right)(\mathfrak{u}) \leq Q_{R}^{V}\left(F_{\psi}\right) .
$$

Combining this with (A.41), we obtain (A.40).

Step 3. Let us assume ${ }^{12}$ that $I_{R}^{V}(\sigma)=0$. Then $\sigma \in \mathcal{P}\left(\mathcal{H}^{r}\right)$ and

$$
0=I_{R}^{V}(\sigma)=\sup _{F \in C_{b}\left(\mathcal{H}^{r}\right)}\left(\langle F, \sigma\rangle-Q_{R}^{V}(F)\right) .
$$

So taking here $F=F_{\psi}$ for any $\psi \in \mathfrak{D}_{+}$and using the result of Step 2, we get

$$
0 \leq \inf _{\psi \in \mathfrak{D}_{+}} \int_{\mathcal{H}^{r}} \frac{\mathbb{L}_{V} \psi}{\psi} \sigma(\mathrm{d} \mathfrak{u}) .
$$

Since $\mathfrak{S}_{t}^{V}$ is a Markov semigroup, we have $\mathbb{L}_{V} \mathbf{1}=\mathbf{0}$. We see that $\theta=0$ is a local minimum of the function

$$
f(\theta):=\int_{\mathcal{H}^{r}} \frac{\mathbb{L}_{V}(1+\theta \psi)}{1+\theta \psi} \sigma(\mathrm{d} \mathfrak{u})
$$

for any $\psi \in \mathfrak{D}_{+}$, so

$$
0=f^{\prime}(0)=\int_{\mathcal{H}^{r}} \mathbb{L}_{V} \psi \sigma(\mathrm{d} \mathfrak{u}) .
$$

Combining this with property (i) in Lemma A.7, we obtain

$$
\int_{\mathcal{H}^{r}} \mathfrak{S}_{t}^{V} \psi \sigma(\mathrm{d} \mathfrak{u})=\int_{\mathcal{H}^{r}} \psi \sigma(\mathrm{d} \mathfrak{u}), \quad t>0
$$

From (ii) in Lemma A.7, we derive that $\sigma$ is a stationary measure for $\mathfrak{S}_{t}^{V}$, and Lemma A.8 implies that $\sigma=h_{V} \mu_{V}$. This completes the proof of Proposition 1.3.

${ }^{12}$ As $I_{R}$ defined by (1.6) is a good rate function, the set of equilibrium measures for $V$ is non-empty. So the set of zeros of $I_{R}^{V}$ is also non-empty, by the remark made at the end of Step 2 of the proof of Theorem 1.1. 
Proof of Lemma A.7. Step 1: Property (i). Let us show that, for any $\psi \in C_{b}\left(\mathcal{H}^{r}\right)$, the function $\varphi_{t}=\mathfrak{S}_{t}^{V, F} \psi$ satisfies the equation in the Duhamel form

$$
\varphi_{t}=\mathfrak{S}_{t}^{V} \psi+\int_{0}^{t} \mathfrak{S}_{t-s}^{V}\left(F \varphi_{s}\right) \mathrm{d} s
$$

Indeed, we have

$$
\begin{aligned}
\varphi_{t}-\mathfrak{S}_{t}^{V} \psi= & \lambda_{V}^{-t} h_{V}^{-1} \\
& \times \mathbb{E}_{\mathfrak{u}}\left\{\exp \left(\int_{0}^{t} V\left(\mathfrak{u}_{\tau}\right) \mathrm{d} \tau\right)\left[\exp \left(\int_{0}^{t} F\left(\mathfrak{u}_{\tau}\right) \mathrm{d} \tau\right)-1\right] h_{V}\left(\mathfrak{u}_{t}\right) \psi\left(\mathfrak{u}_{t}\right)\right\} .
\end{aligned}
$$

Integrating by parts and using the the Markov property, we get

$$
\begin{aligned}
\varphi_{t}-\mathfrak{S}_{t}^{V} \psi= & \lambda_{V}^{-t} h_{V}^{-1} \\
& \times \int_{0}^{t} \mathbb{E}_{\mathfrak{u}}\left\{\exp \left(\int_{0}^{t} V\left(\mathfrak{u}_{\tau}\right) \mathrm{d} \tau\right)\left[F\left(\mathfrak{u}_{s}\right) \exp \left(\int_{s}^{t} F\left(\mathfrak{u}_{\tau}\right) \mathrm{d} \tau\right)\right] h_{V}\left(\mathfrak{u}_{t}\right) \psi\left(\mathfrak{u}_{t}\right)\right\} \mathrm{d} s \\
= & \int_{0}^{t} \lambda_{V}^{-s} h_{V}^{-1} \mathbb{E}_{\mathfrak{u}}\left\{\exp \left(\int_{0}^{s} V\left(\mathfrak{u}_{\tau}\right) \mathrm{d} \tau\right) h_{V}\left(\mathfrak{u}_{s}\right) F\left(\mathfrak{u}_{s}\right) \varphi_{t-s}\left(\mathfrak{u}_{s}\right)\right\} \mathrm{d} s \\
= & \int_{0}^{t} \mathfrak{S}_{s}^{V}\left(F \varphi_{t-s}\right) \mathrm{d} s=\int_{0}^{t} \mathfrak{S}_{t-s}^{V}\left(F \varphi_{s}\right) \mathrm{d} s .
\end{aligned}
$$

This proves (A.42). The identity

$$
\mathfrak{S}_{t}^{V}\left(\varphi_{s}\right)(\mathfrak{u})=\varphi_{s+t}(\mathfrak{u})=\varphi_{s}(\mathfrak{u})+\int_{0}^{t} \mathfrak{S}_{\tau}^{V}\left(\mathfrak{S}_{s}^{V} g\right)(\mathfrak{u}) \mathrm{d} \tau, \quad t \geq 0, \mathfrak{u} \in \mathcal{H}^{r}
$$

shows that $\varphi_{s} \in \mathfrak{D}\left(\mathbb{L}_{V}\right)$ for $\psi \in \mathfrak{D}\left(\mathbb{L}_{V}\right)$ and $r>0$.

Step 2: Property (ii). Assume that, for some $\sigma_{1}, \sigma_{2} \in \mathcal{P}\left(\mathcal{H}^{r}\right)$, we have

$$
\left\langle\psi, \sigma_{1}\right\rangle=\left\langle\psi, \sigma_{2}\right\rangle, \quad \psi \in \mathfrak{D}_{+} .
$$

Let us take any $\psi \in C_{b}\left(\mathcal{H}^{r}\right)$ such that $c \leq \psi(\mathfrak{u}) \leq C$ for any $\mathfrak{u} \in \mathcal{H}^{r}$ and some constants $c, C>0$. Then $\tilde{\varphi}_{s}:=$ $\frac{1}{s} \int_{0}^{s} \mathfrak{S}_{\tau}^{V} \psi \mathrm{d} \tau$ belongs to $\mathfrak{D}_{+}$for any $s>0$. Indeed, the inequality $c \leq \tilde{\varphi}_{r}(\mathfrak{u}) \leq C$ follows immediately from the definition of $\mathfrak{S}_{S}^{V}$, and the fact that $\tilde{\varphi}_{s} \in \mathfrak{D}\left(\mathbb{L}_{V}\right)$ follows from the identity

$$
\begin{aligned}
\mathfrak{S}_{t}^{V} \tilde{\varphi}_{s}-\tilde{\varphi}_{s} & =\frac{1}{s} \int_{0}^{s}\left(\mathfrak{S}_{\tau+t}^{V} \psi-\mathfrak{S}_{\tau}^{V} \psi\right) \mathrm{d} \tau=\frac{1}{s} \int_{s}^{s+t} \mathfrak{S}_{\tau}^{V} \psi \mathrm{d} \tau-\frac{1}{s} \int_{0}^{t} \mathfrak{S}_{\tau}^{V} \psi \mathrm{d} \tau \\
& =\int_{0}^{t} \mathfrak{S}_{\tau}^{V}\left(\frac{\mathfrak{S}_{s}^{V} \psi-\psi}{s}\right) \mathrm{d} \tau .
\end{aligned}
$$

Then, by (A.43), we have

$$
\left\langle\tilde{\varphi}_{s}, \sigma_{1}\right\rangle=\left\langle\tilde{\varphi}_{s}, \sigma_{2}\right\rangle, \quad r>0 .
$$

Using the continuity of the mapping $s \mapsto \mathfrak{S}_{S}^{V} \psi(\mathfrak{u})$ from $\mathbb{R}_{+}$to $\mathbb{R}$, we see that $\tilde{\varphi}_{s}(\mathfrak{u}) \rightarrow \psi(\mathfrak{u})$ as $s \rightarrow 0$. Passing to the limit in (A.44) and using the Lebesgue theorem on dominated convergence, we obtain $\left\langle\psi, \sigma_{1}\right\rangle=\left\langle\psi, \sigma_{2}\right\rangle$. It is easy to verify that the set $\left\{\psi \in C_{b}\left(\mathcal{H}^{r}\right): \inf _{\mathfrak{u} \in \mathcal{H}^{r}} \psi(\mathfrak{u})>0\right\}$ is determining, so we get $\sigma_{1}=\sigma_{2}$. 


\section{Proof of Lemma 5.2}

Let us first prove (5.13). We take $p_{4}=6 /(1+2 r)$ the maximal exponent for which the Sobolev embedding $H^{1-r} \hookrightarrow$ $L^{p_{4}}$ holds. We choose $p_{2}$ in such a way that exponents $\left(p_{i}\right)$ are Hölder admissible. It follows that $p_{2}=6 /(5-\rho-$ $2 r-3 \varkappa)$. Now let $\varkappa>0$ be so small that $\rho+2 r \varkappa \leq 2$. Then a simple calculation shows that $(1-\varkappa) p_{2} \leq 6 /(3-2 r)$, so the Sobolev embedding implies the first inclusion in (5.13).

We now prove (5.14). Proceeding as above, we take $q_{4}=6 /(1+2 r)$ and choose $q_{2}$ such that the exponents $\left(q_{i}\right)$ are Hölder admissible, i.e., $q_{2}=6(\rho+2) /(12-(\rho+2)(1+2 r+3 \varkappa))$. It is easy to check that for $\varkappa<1 / 2-r$, we have $(1-\varkappa) q_{2} \leq 6$. The Sobolev embedding allows to conclude.

\section{Proof of Lemma 5.3}

In view of inequality (5.19), we have

$$
\begin{aligned}
\beta^{-1} \frac{\mathrm{d}}{\mathrm{d} t}(1+x)^{\beta} & =(1+x)^{\beta-1} \dot{x} \leq(1+x)^{\beta-1}\left(-\alpha x+g x^{1-\beta}+b\right) \\
& \leq-\alpha x(1+x)^{\beta-1}+g+b \leq-\frac{\alpha}{2} x^{\beta}+\alpha+g+b .
\end{aligned}
$$

Fixing $t \in[0, T]$ and integrating this inequality over $[0, t]$, we obtain

$$
\beta^{-1}(1+x(t))^{\beta}+\frac{\alpha}{2} \int_{0}^{t} x^{\beta}(\tau) \mathrm{d} \tau \leq \beta^{-1}(1+x(0))^{\beta}+\int_{0}^{t}(\alpha+g(\tau)+b(\tau)) \mathrm{d} s,
$$

which implies (5.20).

\section{Acknowledgements}

The authors thank the referees for their careful reading and valuable comments. The authors thank Armen Shirikyan for helpful discussions. The research of DM was carried out within the MME-DII Center of Excellence (ANR 11 LABX 0023 01) and partially supported by the ANR grant STOSYMAP (ANR 2011 BS01 015 01). The research of VN was supported by the ANR grants EMAQS (No. ANR 2011 BS01 017 01) and STOSYMAP (No. ANR 2011 BS01 015 01).

\section{References}

[1] T. Bogenschütz and A. Doebler. Large deviations in expanding random dynamical systems. Discrete Contin. Dyn. Syst. 5 (4) (1999) 805-812. MR1722372

[2] W. Bryc and A. Dembo. Large deviations and strong mixing. Ann. Inst. Henri Poincaré Probab. Stat. 32 (4) (1996) 549-569. MR1411271

[3] G. Da Prato and J. Zabczyk. Stochastic Equations in Infinite Dimensions. Cambridge University Press, Cambridge, 1992. MR1207136

[4] A. de Acosta. Upper bounds for large deviations of dependent random vectors. Z. Wahrsch. Verw. Gebiete 69 (4) (1985) 551-565. MR0791911

[5] A. Dembo and O. Zeitouni. Large Deviations Techniques and Applications. Springer-Verlag, Berlin, 2000. MR2571413

[6] M. D. Donsker and S. R. S. Varadhan. Asymptotic evaluation of certain Markov process expectations for large time, I-II. Comm. Pure Appl. Math. 28 (1975) 1-47, 279-301. MR0386024

[7] M. Gourcy. A large deviation principle for 2D stochastic Navier-Stokes equation. Stochastic Process. Appl. 117 (7) (2007) $904-927$. MR2330725

[8] M. Gourcy. Large deviation principle of occupation measure for a stochastic Burgers equation. Ann. Inst. Henri Poincaré Probab. Stat. 43 (4) (2007) 441-459. MR2329511

[9] H. Hennion and L. Hervé. Limit Theorems for Markov Chains and Stochastic Properties of Dynamical Systems by Quasi-Compactness. Lecture Notes in Math. 1766. Springer-Verlag, Berlin, 2001. MR1862393

[10] V. Jakšić, V. Nersesyan, C.-A. Pillet and A. Shirikyan Large deviations and mixing for dissipative PDE's with unbounded random kicks. Preprint, 2014.

[11] V. Jakšić, V. Nersesyan, C.-A. Pillet and A. Shirikyan. Large deviations and Gallavotti-Cohen principle for dissipative PDEs with rough noise. Comm. Math. Phys. 336 (1) (2015) 131-170. MR3322369

[12] V. Jakšić, V. Nersesyan, C.-A. Pillet and A. Shirikyan. Large deviations from a stationary measure for a class of dissipative PDEs with random kicks. Comm. Pure Appl. Math. 68 (12) (2015) 2108-2143. MR3417879

[13] V. Jakšić, Y. Ogata, Y. Pautrat and C.-A. Pillet. Entropic fluctuations in quantum statistical mechanics - an introduction. In Quantum Theory from Small to Large Scales, J. Frhlich et al (Eds). Oxford University Press, Oxford, 2012. 
[14] Y. Kifer. Large deviations in dynamical systems and stochastic processes. Trans. Amer. Math. Soc. 321 (2) (1990) 505-524. MR1025756

[15] I. Kontoyiannis and S. P. Meyn. Spectral theory and limit theorems for geometrically ergodic Markov processes. Ann. Appl. Probab. 13 (1) (2003) 304-362. MR1952001

[16] S. Kuksin and V. Nersesyan. Stochastic CGL equations without linear dispersion in any space dimension. Stoch. Partial Differ. Equ., Anal. Computat. 1 (3) (2013) 389-423. MR3327512

[17] S. Kuksin and A. Shirikyan. Ergodicity for the randomly forced 2D Navier-Stokes equations. Math. Phys. Anal. Geom. 4 (2) (2001) 147-195. MR1860883

[18] S. Kuksin and A. Shirikyan. Coupling approach to white-forced nonlinear PDEs. J. Math. Pures Appl. (9) 81 (6) (2002) 567-602. MR1912412

[19] S. Kuksin and A. Shirikyan. Mathematics of Two-Dimensional Turbulence. Cambridge University Press, Cambridge, 2012. MR3443633

[20] A. Lasota and T. Szarek. Lower bound technique in the theory of a stochastic differential equation. J. Differential Equations 231 (2) (2006) 513-533. MR2287895

[21] D. Martirosyan. Exponential mixing for the white-forced damped nonlinear wave equation. Evol. Equ. Control Theory 3 (4) (2014) 645-670. MR3274652

[22] D. Martirosyan. Large deviations for stationary measures of stochastic nonlinear wave equation with smooth white noise. Comm. Pure Appl. Math. 70 (9) (2017) 1754-1797.

[23] J. C. Mattingly. Exponential convergence for the stochastically forced Navier-Stokes equations and other partially dissipative dynamics. Comm. Math. Phys. 230 (3) (2002) 421-462. MR1937652

[24] C. Odasso. Exponential mixing for stochastic PDEs: The non-additive case. Probab. Theory Related Fields 140 (1-2) (2008) $41-82$. MR2357670

[25] L. Rey-Bellet and L.-S. Young. Large deviations in non-uniformly hyperbolic dynamical systems. Ergodic Theory Dynam. Systems 28 (2) (2008) 587-612. MR2408394

[26] A. W. Roberts and D. E. Varberg. Convex Functions. Academic Press, New York, 1973. MR0442824

[27] L. Wu. Large and moderate deviations and exponential convergence for stochastic damping Hamiltonian systems. Stochastic Process. Appl. 91 (2) (2001) 205-238. MR1807683 